\title{
LEBEUF
}

\section{ARBRES FRUITIERS}




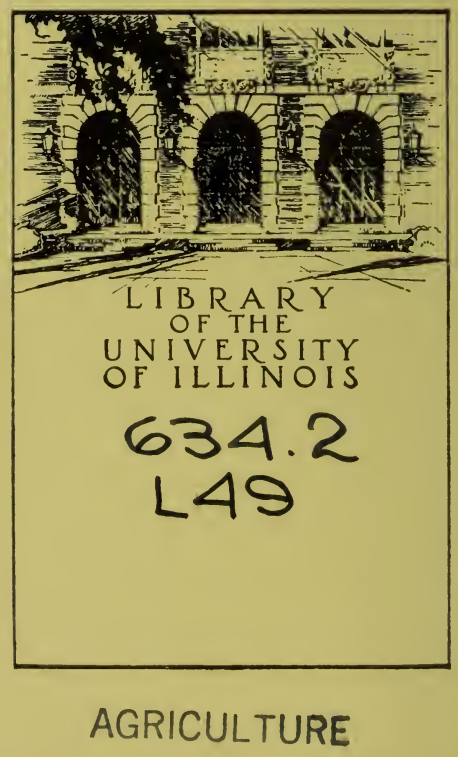







\section{GULTURE ET TAILLE}

RATIONNELLES ET ÉGONOMIQUES

$$
\text { I) E S }
$$

ARBRES FRUITIERS 



\title{
ARBRES FRUITIERS
}

\author{
GULTURE ET TAILLE
}

RATIONNELLES ET ÉCONOMIQUES

DES

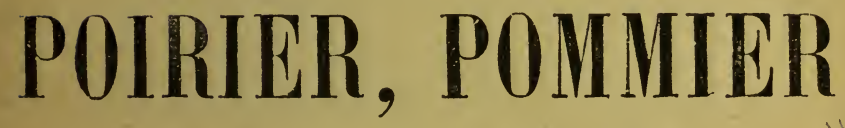

\section{PRUNIER, CERISIER}

○ U

10 MoYens de PRÉPARer Le SOL ET DE PLANTER ÉCoNOUIQUEMENT POUR AVUIR DES ARBIES PRODUCTIFS ET DE LOVGUE DUREE

20 IESCILIPTION DES 30 MEILLEULES VARIÉTÉS DE POIRES POUR ESPALIERS

ET DES 30 PLUS MÉritantes I'OUR HAUTE. TIGE POCU LA CONSOMMATION DE L'ÉTÉ, LE L'AUTOMNE, DE L'HIVER ET DU PRINTEMPS

30 FORMES NOUVELLES NATURELLE OPPOSÉES AUX FORMES THÉORIQUES

ET FANTAISISTES IMPRODUCTIVES ET ONÉRECSES

40 TAILLE SIMPLIFIÉE; 5o CONSERVATION DES FBUITS

$6^{\circ}$ EXTINCTION DES VARIÉTÉS ANCIENNES ET LEUR REMPLACEMENT

7 SILHOULTTES OU GRAVURES DES 43 MEILLELRES POILES DE GRANDEUR NATUIIELI E

ET GRAVÉES D'APRÉS NATURE

UX ESPALIER Er UNE PYRAMIDE MODÈLES, FTC.

P A R

\section{V.-F. LEBEUF}

\section{PARIS}

GHEZ M. SAGNIER, LIBRAIRE

7, GARREFOUR DE L'ODÉON, 7 



\section{2 \\ UNIVERSITY OF ILLINOIS \\ AGRICULTURE LIBRARY}

POURQUOI UN LIVRE NOUVEAU

SUR LES ARBRES FRUITIERS

Après la Quintinie et tant d'autres arboriculteurs anciens, après Dalbret et Hardy, ces professeurs modernes dont le mérite est reconnu, il n'y a plus rien à dire sur la taille et la conduite des arbres fruitiers; aussi n'est-ce pas un ouvrage spécial sur la taille que nous offrons aux amateurs de fruits et aux spéculateurs. Le but de ce petit traité est de démontrer :

$1^{\circ}$ Que les frais considérables résultant du mode de plantation recommandé par les arboriculteurs modernes, sont faits en pure perte ;

$2^{\circ}$ Que ces frais sont nuisibles aux plantations en ce que, non-seulement ils en causent la ruine, mais parce qu'ils empêchent le cultivateur de s'adonner à la culture fruitière ;

$3^{\circ}$ Que les formes auxquelles les arbres fruitiers sont soumis, notamment le poirier, sont sinon impossibles à réaliser, du moins à maintenir ; qu'elles sont très-onéreuses, contre nature, peu productives, et conduisent rapidement l'arbre à la mort;

a

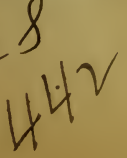


$4^{\circ} Q u^{\prime} i l$ est indispensable de connaître les meilleures variétés à cultiver.

Indépendamment de ces démonstrations, nous donnons la description des meilleures poires, pour guider le planteur et l'amateur qui sont souvent embarrassés pour faire un bon choix. - Nous y avons ajouté la liste des fruits les plus recommandables, en pommes, prunes, etc.

Un traité succinct de taille simplifiée et raisonnée complète ce travail et dispensera nos lecteurs de recourir à d'autres livres.

Nous pensons donc que cet ouvrage comblera une lacune réelle, et qu'il rendra des services à ceux qui ne sont pas versés dans la culture des arbres fruitiers.

\section{V.-F. LEBEUF.}

Argenteuil, le $1^{\text {er }}$ décembre 1869. 


\section{PREMIERE PARTIE}

\section{GULTURE DES ARBRES FRUITIERS}

PLANTATION, CHOIX DES VARIÉtéS A GULTIVER, ETC.

INSUFFISANCE DE LA PRODUCTION FRUITIÈRE EN FRANCE

On a beaucoup planté, depuis un demi-siècle, et cependant la production est loin de satisfaire aux besoins. A part quelques contrées, où les arbres fruitiers sont cultivés en plein champ, comme la Normandie qui en tire sa boisson, partout ailleurs, les fruits n'entrent que pour une proportion insignifiante dans l'alimentation.

Les grandes villes absorbent la majeure partie de la production et offrent des débouchés insatiables à toutes espèces de fruits, bien que la qualité de ceux qu'on y amène laisse beaucoup à désirer.

Paris consomme, à ce qu'il paraît, 10 millions de kilogrammes de fruits frais, fraises, raisins, pommes, 
poires, prunes, cerises, abricots, etc. Ce qui, pour une population de 1 million 600,000 habitants, fait en nombre rond 6 kilos par individu. Dans certaines villes la consommation est un peu plus forte, dans d'autres elle l'est moins. Dans les localités favorisées elle peut être évaluée à 20 kilos par tête. On voit que nous ne produisons pas ce qui est nécessaire à une consommation ordinaire.

Jusqu'à présent on s'est peu occupé de produire de bons fruits et surtout des fruits de garde; de telle sorte qu'il faut qu'ils soient consommés de suite. S'ils étaient de meilleure qualité et de plus longue garde, on en consommerait davantage.

L'étranger vient nous enlever une grande partic des fruits qui peuvent se transporter facilement ; l'exportation serait donc d'autant plus importante que les marchés seraient mieux et plus longtemps approvisionnés.

Souvent nous avons entendu dire : " Si tout le monde plante, on ne saura plus que faire de ses produits; il faudra les vendre à vil prix. „C'est là une grosse erreur I Plus la production est grande, plus la consommation augmente, et les prix ne s'abaissent pas quand le produit est de bonne qualité. On produit, aujourd'hui, dix fois autant de fruits qu'il y a quarante ans. Est-ce que les prix ont diminué? Est-ce que le consommateur a fait défaut? D'ailleurs les voies de circulation, les moyens de transport se multiplient et favorisent l'exportation de plus en plus.

On a dit, encore, qu'augmenter les plantations, co serait diminuer les récoltes de blé. Lit quand cela 
scrait, qu'en résulterait-il? Est-ce que la poire ne vaut pas le pain ? Qu'importe au producteur? Ce qu'il doit chercher avant tout, n'est-ce pas de faire rapporter à sa terre le plus possible ? Or, que lui rapporte le blé ? Rien! Que lui rapporteraient donc les fruits? Trois ou quatre mille francs par hectare. Le cultivateur est un fabricant; s'il abandonne un produit qui ne lui rapporte rien pour un autre plus profitable, c'est son droit: lui d'abord et avant tout.

Notre sol est surtout propre à la production du vin et des fruits à pepins : il l'est fort peu à celle du blé, comparativement à d'autres contrées de l'étranger. Faisons donc de la vigne et des fruits, puisque nulle part on ne peut nous faire concurrence, et ne faisons du blé que quand nous ne pourrons nous en dispenser; il nous restera encore trop de terres à lui consacrer. Nous ne manquerons pas de pain pour cela : l'Égypte, la Russie et d'autres pays nous en fourniront.

L'insuffisance des plantations provient de ce qu'on croit généralement qu'il est d'absolue nécessité d'avoir des terrains essentiellement bons pour planter; le paysan, alors, ne consent pas à sacrifier le meilleur coin de ses terres. Ensuite, il faut faire des frais d'achats de sujets; puis attendre dix ans avant de récolter. Avec des soins et de l'intelligence, on päre à tous ces inconvénients qui ne sont qu'apparents comme on le verra plus loin.

Si l'habitant des campagnes ne manquait pas de l'instruction la plus élémentaire, il y aurait à espérer qu'il sortirait de l'état d'insouciance où il est; mal- 
heureusement il est esclave de la routine et de l'ignorance, et il n'aura pas de longtemps la force, le savoir et le pouvoir de se tirer de l'ornière où il végète. En Allemagne, en Angleterre, il y a une certaine instruction au sein des masses les plus éloignées des grands centres; tandis qu'à la porte même des villes nous voyons les cultivateurs français aussi primitifs que si l'art de lire et d'écrire était encore à inventer. Que faut-il attendre de pareils cultivateurs? Nous sommes en arrière sur presque toutes les nations de l'Europe. Que les hommes capables se mettent à l'œuvre sans crainte: ils ont le champ libre. Les plantations qu'ils feront leur rapporteront un bénéfice considérable; ils donneront ainsi l'exemple à leurs concitoyens qui finiront par les imiter et trouver le bien-être là où ils n'ont que misère. Les planteurs intelligents qui ouvriront la marche, feront, à la fois, une bonne action et une spéculation profitable à eux et aux autres.

DU POIRIER

Le poirier est le premier des arbres fruitiers. Aucun autre ne donne des fruits aussi succulents, aussi agréablement parfumés, aussi variés et d'une aussi longue garde.

C'est à tort que quelques pomologistes ont proclamé le calville blanc le roi des fruits. Si cette pomme a le mérite de se conserver aussi longtemps 
que la poire, elle n'en a ni la finesse, ni l'abondance d'eau, ni la fraîcheur.

Non-seulement le poirier se recommande par la qualité de ses fruits, mais encore par la fäcilité avec laquelle il se plie à toutes les formes. Il réussit en plein vent, en espalier, en pyramides, en cordons, en touffes, etc. Son port est de toute beauté comme arbre à haute tige, et, comme espalier, il est admirable.

On devrait le rencontrer partout, dans les champs, comme darıs les jardins et dans les vergers. Nul arbre n'est aussi propre que le poirier à donner de riches et abondantes récoltes. Ses fruits obtiennent, quand ils sont choisis avec discernement, un prix bien supérieur à celui des pommes. Il est donc de toute importance que l'on s'attache à faire un choix judicieux des bonnes espèces et à les cultiver à l'exclusion de toutes les autres qui n'offrent d'autre intérêt que celui de la curiosité.

On a reproché au poirier d'être plus difficile sur le sol que le pommier; c'est encore une erreur, car si quelques variétés se montrent rebelles à certains terrains, il y en a un grand nombre qui réussissent là où ne réussit pas le pommier.

On a dit aussi qu'il se mettait à fruit plus tard que le pommier. Ce reproche n'est guère plus fondé que le précédent. D'ailleurs, quand mème il serait vrai, il ne serait pas suffisant pour faire donner la préférence au pommier dont les fruits ont cinq ou six fois moins de valeur. 
DU SOL PROPRE AU POIRIER

Le poirier réussit dans tous les sols, qu'ils soient calcaires ou siliceux, pourvu que la couche de terre végétale soit assez épaisse ou que le sous-sol ne soit pas imperméable aux racines et ne retienne pas l'eau à la surface.

La plupart des auteurs prétendent qu'il faut au p oirier un bon sol, substantiel, profond et qu'il ne réussit pas dans ceux dont la couche arable est très-légère et sèche. Nous avons vu de magnifiques poiriers dans des terres de cette nature, dont le sous-sol était rocailleux, argilo-calcaire-ferrugineux. Les plus beaux que nous ayons vus étaient plantés dans un sol sablonneux de 50 centimètres seulement de profondeur, sous lequel se trouvait une terre de Loam également sablonneuse. Les poiriers de Rousselet mesuraient près de 2 mètres de circonférence et 16 à 18 mètres de hauteur. Tous les ans ils se chargeaient d'une quantité innombrable de fruits. Le propriétaire, qui était notre parent, nous a assuré que ces arbres (il y en avait 4 de la même variété) avaient produit, pendant plus de 20 ans, en moyenne, 4 à 5,000 fruits chacun.

Le poirier se plait encore et donne d'excellents réjultats dans les terres ferrugincuses dont le sous-sol st formé de roches qui laissent entre elles de larges in terstices remplis de bonne terre à une profondeur de 80 centimètres à 1 mètre. Les arbres trouvent 
dans ces sols une fraîcheur constante, les racines s'emparent de tous les vides existants entre les roches et s'assoient très-solidement. Généralement, ces arbres sont très-productifs et les fruits de bonne qualité; une fois noués, ils sont presque toujours assurés.

Les terres franches, les terres d'alluvion, les sables gras lui conviennent également. Les terrains légers calcaires ou siliccux à sous-sol frais sont aussi très-convenables au poirier quand la couche végétale est assez profonde, le sous-sol perméable et non aride. On le cultive avec succès aussi dans les terres argileuses qui conservent l'eau à 50 centimètres de la superficie du sol, en employant le drainage. Il réussit dans toute la France, si ce n'est dans le Midi dont il redoute les chaleurs excessives et les vents desséchants, le mistral. Dans les parties les plus chaudes, on peut encore en obtenir des récoltes en le plantant à l'exposition du nord.

On peut donc poser, en principe, que le poirier réussit presque dans tous les sols, en choisissant le sujet qui doit recevoir la greffe, c'est-à-dire en le greffant sur coignassier ou sur franc, suivant les besoins.

LE MODE ACTUEL DE PLANTATION EST VICILUA.

Si vous consultez tous les livres qui traitent de la plantation des arbres fruitiers, vous y lirez qu'il faut défoncer le sol à 1 mètre de profondeur. 
Si vous consultez les planteurs, vous apprendrez que la plupart se bornent à ouvrir des trous de 50 centimètres carrés sur 450 ou 50 centimètres de profondeur et souvent moins.

Ces deux modes d’opérer sont à peu près les seuls employés; ils sont aussi mauvais l'un que l'autre.

En défonçant à 1 mètre de profondeur que fait-on? La plupart du temps on ramène à la superficie un sol infertile, ou des pierres, ou du sable, et le terrain est perdu à tout jamais, à moins que le sous-sol ne soit végétal jusqu'à cette profondeur, auquel cas le défoncement est à peu près inutile.

Les arbres plantés dans un tel sol marchent trèsrapidement; ils ont une végétation luxuriante, surtout si l'on a fumé copieusement comme on le recommande. Pendant les six ou huit premières années, la végétation se soutient; mais il arrive un moment où les racines ont envahi tout le sol remué, elles ont acquis une avidité de végétation que rien ne satisfait; elles continuent à s'étendre en tous sens, et quand elles rencontrent le sol ferme, aride, dur, ou non mélangé, non défoncé, les spongioles s'émacient, se ferment, s'anéantissent, la nourriture manque et la végétation s'arrête forcément. L'arbre, jusque-là plein de vigueur, commence à souffrir ; il se met à fruit, il languit, dépérit et meurt. Voilà ce qui se passe quatre fois sur cinq.

Nous avons vu des pêchers, des poiriers, plantés en espalier, après un défoncement et un apport de terre, domner des résultats prodigieux de végéta- 
tion pendant 8 ou 10 ans; il n'était pas rare de voir des scions de 3 mètres de longueur. Eh bien, à 16 ou 18 ans; il n'y en avait plus un seul de vivant sur près de 100 que comptait la plantation.

En ouvrant des trous de 50 centimètres cubes, qu'arrive-t-il? Dès la seconde année, les racines ont envahi la terre meuble et leurs spongioles ne peuvent plus s'étendre et fonctionner avec la même facilité. Il faut qu'elles se frayent un passage dans un sol dur; de là, ralentissement dans la production de la sève et des rameaux : la végétation marche avec peine. Pour que les racines se façonnent à un sol non remué, et presque neuf, il faut plusieurs années. Les branches à bois prennent peu de force, les brindilles, les dards abondent, l'arbre se met prématurément à fruit et il n'atteint qu'un développement très-restreint, parce que la sève qui devait être utilisée à la production des branches à bois se trouve absorbée par les fruits. On n'obtient donc encore que des arbres rabougris ou de petite dimension et d'une durée éphémère.

On reconnaîtra avec nous, que ni l'un ni l'autre de ces deux modes de plantation n'est recommandable, et qu'ils constituent des opérations contre nature.

Non-seulement on fait mal les plantations, mais on ne se préoccupe pas de la valeur des fruits. On plante un poirier et c'est tout : que ce soit une duchesse ou un catillac, un doyenné de juillet ou un passe-colmar, peu importe. Si encore on s'en tenait à des variétés de ce mérite; mais pas du tout. On 
plante dis arbres sans nom, et souvent mème on greffe sauvageon sur sauvageon.

Tient-on compte des différences qui doivent exister dans le choix des terres, selon qu'on plante une variété greffée sur coignassier ou sur franc? Aucunement. C'est à peine si le planteur sait distinguer l'une de l'autre.

Combine-t-on les plantations de manière à avoir des fruits le plus longtemps possible? Non. Les uns n'ont que des poires d'été, les autres des poires d'automne. Quant aux poires d'hiver, il n'y en a pas.

Et les poires à cuire sont-elles distinguées des poires à couteau? Pas davantage.

$\mathrm{Si}$, au moins, on tenait compte de celles qui offrent le plus d'avantage à la vente. Du tout, il semble que l'on doive s'en rapporter au hasard.

Tant que l'on plantera sans savoir ce que l'on fait, il n'y aura rien à espérer. Il faut qu'on se persuade bien que le poirier demande une étude sérieuse quoique facile, tant sous le rapport du choix des sujets que sous celui des variétés. Que l'on noublie pas surtout qu'une mauvaise variété coûte autant qu'une bonne, et que son produit est insignifiant. Une poire de choix obtient un prix souvent trèsélevé : ainsi, sur le marché, à Paris, il n’est pas rare de voir des poires à 2 fr. $\check{0} 0$ le 100, à côté d'autres qui se placent très-facilement à 60,80 et 100 francs le 100 . 
LES DÉEONGEMENTS PROFONDS OCGASIONNENT DES FRAIS CONSIDÉRABLES SANS COMPENSATION

Ceux qui ont conseillé de défoncer le sol à 1 mètre de profondeur se sont, sans aucun doute, adressés à des millionnaires; car s'ils s'étaient rendu compte de ce qu'il en coûte pour défoncer à ce prix un hectare de terre, ils se seraient aperçu que peu de propriétaires peuvent disposer d'un capital aussi énorme et attendre environ 8 ans sans en retirer une rémunération satisfaisante, Qu'un riche propriétaire établisșe une plantation de quelques ares dans ces conditions, rien de mieux; il dépensera quelques milliers dẹ francs et tout sera dit; mais qu'on pose en principe de faire des défoncements semblables, qu'on fume fortement, qu'on épierre, etc., etc., autant vaudrait proposer à un spéculateur de brûler sa maison.

Pour défoncer à 1 mètre, il en coûterait pour l'hectare, soit pour 10,000 mètres à 1 franc le mètre, épierrage compris, 10,000 francs, ci . . . 10,000

Transport de pierres, nivellement, etc., en moyenne...........

$$
\text { Ensemble. .... } \overline{10,400}
$$

Intérêts non cumulés pendant 8 ans à 5 p. $100 \ldots \ldots \ldots, \frac{4,160}{14,560}$

14,560 fr anncs, sans compter ies frais de planta- 
tion, l'achat des arbres et la fumure qui devient indispensable avec un pareil défoncement, etc. !...

On nous objectera, sans nul doute, que le défoncement peut se faire à 75 centimètres, peut-être à moins; ce qui réduirait les frais à $7 \check{000}$ fr. ; c'est possible, mais il est à craindre que plus souvent encore ce travail soit compliqué de l'extraction de roches et d'incidents imprévus qui contrebalanceront les circonstances favorables sur lesquelles on se fonderait pour réduire le prix ci-dessus.

Ainsi donc, à la neuvième année, on arrive à avoir à supporter les intérêts d'une somme de 14,506 fr., provenant du fait seul du défoncement, ce qui grève annuellement la récolte de fr. 728. Quel est le planteur assez hardi, assez peu soucieux de ses intérêts pour se résigner à faire cette dépense? S'il n'y en a.pas, ce que nous croyons fermement, à quoi bon ces instructions aussi inutiles qu'impraticables?

LES DEEONCEMENTS PROFONDS SONT-ILS DTILES? A QUELLE PROFONDEUR FAUT-IL DÉFONGER?

Vous avez sans doute remarqué dans les forêts assises sur des sols d'une fertilité très-ordinaire ces énormes chênes, ces hêtres gigantesques, ces fruitiers immenses. Est-ce que le terrain qui a reçu le gland, la faîne, le pepin ou la cerise, a été défoncé? Non certainement! Cependant, vous voyez 
que ces arbres n'ont pas de pendants dans la culture. Les cerisiers, les pommiers, les poiriers que vous plantez ne sont que de misérables pygmées à côté de ces géants.

Avez-vous vu dans les vergers des anciens châteaux, ces vieilles plantations qui datent de plusieurs siècles ? Que dites-vous de ces arbres qui ont survécu à toutes les tempêtes? Cependant la plupart ont été plantés sur un simple défoncement de 60 centimètres : l'inspection seule du terrain l'indique.

Nous avons vu des arbres plantés sur défoncement profond, il y a 60 ans, dans un sol de première qualité, à côté de vieux arbres de même nature. Ils sont morts et les vieux vivent encore.

Nous avons vu planter des pêchers, des poiriers dans un sol défoncé à $\mathbf{1}^{\mathrm{m}}, \breve{0} 0$; on avait rapporté les terres les plus riches, on avait ouvert des tranchées de 4 mètres de large à partir du mur: douze ans après, ils étaient morts. $\mathbf{A}$ deux pas de là, il y avait encore des squelettes de pêchers de 90 ans qui produisaient des fruits succulents. Il y a 25 ans de cela; peut-être y sont-ils encore.

Nous pourrions citer des centaines d'exemples de cette nature, altestant que la culture moderne n'a produit souvent que des avortons, des arbres d'une durée éphémère. La manie de ce siècle est de substituer le raisonnement et la science à la raison et à la pratique.

On nous objectera que les chênes, les hêtres, les fruiliers qu'on rencontre dans les forêts sont des 
arbres sauvages. Sans doute! Est-ce que la nature a deux modes d'action, deux modes de faire? Ce serait absurde de le penser. Nous ne prétendons pas que nos arbres fruitiers doivent atteindre la taille des chênes; mais au moins celle de leurs congénères. Transportez ces chênes, ces fruitiers dans vos jardins; plantez-les dans un sol défoncé à $1^{\mathrm{m}}, \breve{0} 0$ et vous verrez qu'ils ne seront plus que des avortons à côtć de ceux restés en place.

Nous aurions besoin d'une autre preuve que nous la prendrions dans les champs de la Normandie où l'on rencontre de si beaux arbres fruitiers plantés sans soins aucuns. Dans toute la France, on trouve des arbres qui furent jadis plantés pour procurer des abris et de l'ombre aux moissonneurs; il y a de cela un siècle et plus. La plupart d'entre eux ont atteint des dimensions colossales et sont encore pleins de vie. Qui oserait dire qu'ils ont été plantés dans un terrain défoncé?

Les défoncements profonds, les défoncoments à 1 mètre ou 80 centimètres sont une erreur d'autant plus inexplicable qu'ils sont contraires à toutes les lois physiologiques. En effet, il n'y a pas un seul arłore qui puise sa nourriture à plus de 60 centimètres de profondeur, parce qu'au delà la terre n'a plus d'action végétative, l'humus n'a plus de solubilité, il n'y a plus d'air, les influences atmosphériques cessent d'exister; les engrais sont en quelque sorte annihilés, 6 ou 7 ans après le défoncement.

Si vous arrachez un arbre quelque gros qu'il soit, 
vous trouverez les racines à 40 ou 50 centimètres environ de profondeur. Le pivot qui existe dans certaines variétés ne concourt absolument pour rien dans la nutrition de l'arbre. Il est là pour servir de point d'appui, de consolidation. C'est par le pivot que l'arbre se fixe au sol, c'est par ses racines traçantes qu'il s'y grippe et qu'il vit. La preuve c'est qu'on peut impunément supprimer le pivot: l'arbre n'en recoit aucune atteinte.

Si les arbres ne vivent que par les racines superficielles, il est évident qu'il est inutile de défoncer au-dessous, car c'est leur donner de la tendance à s'enfoncer davantage et les forcer de s'éloigner des influences atmosphériques et des causes qui leur donnent la vie.

Il est du reste bien reconnu que les couches inférieures de la terre sont peu riches en substances végétatives et qu'elles sont rapidement épuisées. C'est ce qui explique le peu de succès qu'ont les plantations faites sur défoncement, quand elles comportent des arbres qui ont de la tendance à pivoter, mais qui ont besoin d'un étage supérieur de racines bien assis, comme la plupart des arbres fruitiers.

Le défoncement profond est donc, sinon nuisible, du moins tout à fait inutile. On ne doit défoncer qu'à 60 ou 70 centimètres au plus; c'est tout ce que rćclame la nature. 
DÉFONCEMENTS RATIONXels ET ÉCONOMIQUeS

On défonce de trois manières : $1^{0}$ à la bèche ; go à la charrue défonceuse; $-3^{\circ}$ à la tournée ou pioche à pique.

DÉFONCEMENT A LA BÊCHE

Pour que le défoncement puisse se faire à la bêche, il faut que le sol ne contienne ni roche, ni grosses pierres.

On commence par ouvrir une tranchée de 1 mètre 50 à 2 mètres de large sur 40 à 50 centinètres de profondeur, et on conduit la terre extraite à l'endroit où l'on doit finir le défoncement; puis on laboure le fond de la jauge si c'est possible. Cela fait, on ouvre la seconde jauge qui sert à remplir.la première, en ayant soin de mélanger la terre le plus possible, c'est-à-dire d'intervertir les couches si la terre végétale a au moins 40 centimètres d'épaisseur. On met alors la couche supérieure au fond de la jauge, et celle du fond au-dessus, pour qu'elle se trouve exposée à l'air. 'On donne un labour au fond de la tranchée et la seconde jauge est termincée. Il n'y a plus qu'à suivre jusqu'au bout. Si le sous-sol est de nature à ne pouvoir être entamé par la bêche, on achève avec la tournée de manière à le remuer à 20 centimètres au plus et 130 au moins. 
On voit que ce travail équivaut à deux labours ordinaires et à une fouille de 15 à 20 centimètres. Il s'exécute à raison de 9 à 10 centimes le mètre, soit pour l'hectare 900 à 1,000 francs, épierrage compris, puisqu'à cette profondeur il est nul. S’il y avait des pierres, la bêche, comme nous l'avons dit, ne pourrait fonctionner. Ce défoncement peut se faire également et au même prix avec la houe plate, à manche court.

Il y a loin du chiffre de 1,000 francs à $10,000 \mathrm{fr}$. que coûte le défoncement à 1 mètre. Le second demimètre coûte beaucoup plus que le premier : c'est là qu'on rencontre des pierres et des terres qui ne se laissent ni entamer ni manier facilement.

DÉFONGEMENT A LA CHARRUE

Le défoncement à la charrue convient dans les tcrres légèrement pierreuses où la bêche ne peut plus servir. Il y. a plusieurs charrues défonceuses. On doit donner la préférence à celle qui entame la tcrre le plus profondément et qui fait le travail le plus parfait. Mais quelle que soit celle qu'on choisisse, il faut que le sol soit remué à 53 ou 60 centimètres de profondeur.

Les unes font le travail d'un seul coup comme la charruc Roquebrune; les autres le font en deux fois ; c'est-à-dire qu'on ouvre une raie avec la charrue ordinaire, et que la charrue fouilleuse creuse ensuite le sillon ouvert. La charrue Roquebrune exige la 
force do 6 chevaux pour creuser à כ̌ centimètres dans une terre de consistance moyenne. Du reste, en choisissant le moment où la terre n'est pas sèche, on pourrait, en faisant d'abord un labour ordinaire, travailler avec 4 chevaux.

Le prix d'un défoncement de cette nature vaut celui de quatre labours ordinaires à peu près. Soit environ 100 à 150 francs ; plus 50 franes pour épierrage et nivellement, soit $200 \mathrm{fr}$. en tout.

DÉFONCEMENT A LA TOURNÉ

Les terrains pierreux, dont le sous-sol ne se laisse pas entamer facilement, ceux qui contiennent de grosses pierres et de la roche nécessitent l'emploi de la tournée ou pioche à pique ou à pointe.

L'opération se pratique de la même manière que pour la bêche; seulement, il faut desceller une couche, la déplacer avec la pelle, et renouveler le travail jusqu'à ce qu'on soit arrivé au fond de la jauge, en employant, suivant les circonstances, tantôt la pioche, tantôt la pique ou pointe.

Les roches, les grosses pierres, sont jetées sur lo sol défoncé. Quand on rencontre de grosses roches on se munit d'un poulain (deux madriers reliés ensemble par des traverses) pour les remonter plus aisíment sur le sol.

Le défoncement à la tournée est un peu plus coûteux que celui de la bêche, mais en général, il ne dépasse guère 1,400 francs à 1,500 francs l'hectare. 
SOLS QUI N'ONT QUE PEU DE PROFONDEUR

Ces sols sont de deux sortes, $1^{0}$ ceux qui ont une couche de terre végétale de moins de 40 centimètres et dont le sous-sol est ou un sable sec et inerte, une arène complétement infertile, ou un tuf aride. $2^{\circ}$ ceux qui n'ayant qu'une couche de terre végétale de 30 à 40 centimètres reposent sur un sous-sol qui n'est pas infertile.

Les sols qui font partie de la première classe sont tout à fait impropres à recevoir des arbres fruitiers, il faut renoncer à y faire des plantations, ce serait des frais faits en pure perte. Cependant, on peut y cultiver des pyramides ou des cordons; dans ce cas on défonce 10 centimètres de l'arène pour donner 50 centimètres, au moins, à la couche végétale. Cette arène est jetée sur le sol et non mélangée si on ne doit pas cultiver l'intervalle des arbres, ce qui est de peu d'importance dans ces sortes de sols, car les récoltes n'y ont pas grande valeur et elles nuisent sensiblement à la plantation.

Quant aux seconds, on peut parfaitement les utiliser. S̈ils n'offrent pas les mêmes ressources que certains autres plus farorisés, il n'en est pas moins vrai qu'on peut en tirer parti avantageusement.

Bien que la couche de terre régétale soit peu profonde, on obtient de bons résultats si le sous-sol a 40 centimètres et est perméable, sans être complétement infertile. Il suffit souvent d'un simple défoncement de 50 centimètres et d'une bonne fumure pour 
lui donner l'activité nécessaire à procurer une boune végétation. On réussit d'autant mieux que ce soussol est plutôt frais que sec, car le poirier redoute plus encore la sécheresse que l'humidité.

Nous avons vu des arbres de toute beauté dans des terrains qui n'avaient que 35 centimètres de terre végétale; mais le sous-sol était composé de torre ferrugineuse-argilo-calcaire, mélangée à des couches de pierres sur une épaisseur d'un niètre environ. Ces terres, qu'on nommo herbues dans la Côted'Or, produisent souvent des arbres gigantesques et des fruits de toute beauté.

COMMENT LES ARBRES VÉGÈTENT DANS UN SOL DÉFONCÉ

ET DANS UN SOL QUI NE L'EST PAS MOYENS DE CORRIGER LES DIFFÉRENCLS

Les arbres plantés dans un sol défoncé et fumé végètent vigoureusement pendant plusieurs années, comme nous l'avons dit plus haut. La nature semble ne s'occuper qu'à produire du bois; aussi voit-on des scions d'une prodigieuse longueur. Les branches à fruits ne se rencontrent nulle part, ce qui fait souvent le désespoir des planteurs. Ce n'est guère qu'à l'âge de dix ou douze ans que la fougue de ces arbres s'arrête et que les productions fruitières apparaissent.

Cette vigueur anormale est due au défoncement. Les racines se sont emparées avidement de tout le sol remué; pendant huit, dix ou douze ans, elles ont vécu de ces trésors amassés à grands frais; mais, 
à cet âge, tout est épuisé. La production du bois diminue ou cesse; l'arbre se met à fruit : l'équilibre est infailliblement rompu entre la production du bois et celle des fruits; le bois ne se renouvelle pas, et les fruits épuisent l'arbre qui bientôt dépérit et meurt.

Ce qui a eu lieu, nous venons de le dire pour la seconde fois : on a forcé l'arbre et il est mort prématurément.

Que se passe-t-il dans les plantations faites sans défoncement?

L'arbre étant placé dans un trou étroit et peu profond, les racines ne trouvent pas de terre meuble pour s'étendre et puiser la nourriture nécessaire à son alimentation générale. Ses branches charpentières se dessèchent ou se durcissent; les racines souffrent et meurent d'inanition; mais la plupart dı temps elles luttent longtemps et ne fournissent qu'une petite quantité de sève qui vient s'éteindre dans des productions fruitières, dards et lambourdes, comme il a été dit plus haut.

La charpente ne peut se former, s'étendre ou se soutenir; les fruits qui naissent en grand nombre absorbent à leur profit le peu de sève qu'il y a et empêchent la production du bois. Alors l'arbre reste chétif ou meurt; parfois, si la variété est vigoureuse et la terre peu dure ou la température très-favorable, les spongioles finissent par la pénétrer, la végétation reprend son cours et l'arbre revient comme subitement à la vie.

Le défoncement profond, le défaut de défoncement amènent donc les mêmes résultats, la 
ruine de l'arbre: l'un plus tôt, l'autre plus tard.

Nous nous demandons comment il se fait que les arboriculteurs qui ont conseillé le défoncement profond, comment les praticiens qui ont planté dans des trous microscopiques n'ont pas reconnu les effets désastreux de ces deux modes d'opérer.

Que certains écrivains du jour, qui font profession d'écrire, l'aient omis, rien d'étonnant; car on écrit avant de savoir, avant d'avoir vu et étudié ; mais ce qui nous étonne, c'est que des hommes capables, riches de science et de pratique, qui ont dù reconnaître ces erreurs ne les aient pas signalées.

En définitive, il résulte de ce que nous avons dit que les arbres plantés sur un terrain défoncé à 1 mètre de profondeur périssent par des causes physiologiques que nous avons énoncées; - que ceux qui sont plantés dans des terrains non défoncés périssent également, mais plus tòt par des causes analogues. Nous avons signalé le mal, il nous reste it indiquer le remède.

Quand un arbre a végété avec vigueur pendant quelques années et que.la végétation semble s'arrìter ou diminuer sensiblement, il faut se hâter de le débarrasser d'une grande partie de ses fruits, tailler court, rabattre les brindilles, diminuer le nombre des productions fruitières, en un mot, favoriser la production du bois et diminuer celle des fruits par tous les moyens. Pendant l'hiver, on déchausse l'arbre à un demi-mètre du trone, sur un mètre de large ; on étend une couche do fumier bien pourri dans cette tranchée et on rejette les terres 
dessus. Au printemps, on paille; puis on bassine les feuilles après chaque journée chaude. Avec ces soins continués un an ou deux, l'arbre franchit la période critique et finit par se rétablir s'il n'est pas dans des conditions tout à fait mauvaises.

L'arbre planté dans un trou étroit et peu profond, ne végétant qu'avec peine, parce qu'il n'a pu se faire assez de racines, exige un traitement analogue à celui que nous venons d'indiquer. Les productions fruitières se forment au dépens du bois ; il faut donc faire prendre une direction contraire à la sève, supprimer tous les fruits sans exception, tailler court, enfin faire ce que nous avons dit plus haut, jusqu'à ce que la végétation soit satisfaisante. On peut aussi défoncer le sol à partir de l'endroit où l'on rencontre les racines. En quelques années, l'arbre reprendra de la vigueur s'il n'a pas souffert trop longtemps, s'il n'a pas durci par suite de l'absence de sève. Il sera bon aussi de faire des incisions longitudinales sur la tige et les branches dont les écorces paraissent sèches et dures pour livrer passage à la sève.

Ceci s'applique aussi bien aux arbres à haute tige qu'aux espaliers et pyramides.

FRAIS ET PRODUITS DES DIFEERENTS MODES DE PLANTATIONS

Ayant parlé du défoncement et de son prix de revient, il est naturel que nous examiniens, avant 
tout, quelles sont les dépenses et les recettes présumées des divers genres de plantations.

Il y a quatre sortes de plantations. Les hautes tiges ou plein vent, les pyramides, les espaliers et les cordons.

HAUTES TIGES OU PLEIN VENT

Les arbres à haute tige doivent être espacés, selon la qualité du terrain, de $\tilde{\jmath}$ à 8 mètres en tous sens. Plus le sol est riche, plus les arbres prennent de développement. Supposons une terre de bonne qualité et espacons les arbres de 7 mètres en tous les sens, l'hectare en contiendra environ 210.

Supposons que nous avons fait le défoncement suivant la méthode rationnelle et dans un sol moyen : voici ce qu'une telle plantation coûterait approximativement :

Défoncement de 1 hectare. . . 1,000

Ouverture de 210 trous à $30 \mathrm{c} . \quad 63$

Achat de 210 arbres à $1^{\mathrm{f}} 2 \breve{3}^{\mathrm{c}}$. . $26250^{\mathrm{c}}$

Transport, faux frais..... 30

Fumier pour paillage. . . . 50

Plantation. . . .... 10

Loyer de la terre. . . . . 200

Plantation d'une haie pour clore la plantation. . . . 150

Ensemble. : 1,765 $3^{\mathrm{c}}$ 
Les dépenses annuelles seront donc comme il suit :

Loyer de la terre. . . . . . 200

Fumier pour paillage. ... . 50

Frais de culture. . . . . 70

Cueillette et faux frais. . . . 2 200

Intérêts des frais de plantatations sur $1,500^{\mathrm{f}}$ environ, à 5 p. 100. . . . . . . .

\section{Total. . . . $64 \mathrm{~g}^{\mathrm{f}}$}

Jusqu'à l'âge de 8 ans, les arbres ne produisent rien; c'est au cultivateur à utiliser le sol pour en . tirer le meilleur parti. Pour cela, il y plantera des asperges ou des légumes qui paieront largement le loyer de la terre. La plantation ne commencera donc à être chargée de ces dépenses que lorsqu'elle produira.

De 8 à 12 ans, elle fera ses frais; mais à partir de cet age la production progressera très - rapidement, et de 17 à 20 ans, chaque arbre donnera en moyenne 200 fruits; ce qui fait, pour 210 arbres 42,000 fruits, à 150 francs le mille, la somme do............ $6,300^{\text {f }}$

Les dépenses annuelles étant de.

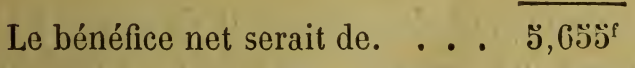

La production, dans la plupart des cas, reste la même jusqu'à l'âge de 50 à 60 ans, après quoi elle diminue. 
PTRAMIDES

Les pyramides doivent être espacées, en moyenne, dans un sol de bonne qualité, de 4 mètres en tous sens; chacune d'elles occupe donc ainsi 16 mètres superficiels, ce qui produit, environ, 600 à l'hectare.

Les frais s'établissent comme il suit :

Défoncement. . . . . . 1,000

Ouverture de 600 trous, à $20 ّ$ c. $\quad 1500$ Achat de 600 pyramides, à $60^{\text {f }}$

le cent. ...... 360

Transport et faux frais..... 25

Fumier pour pailler..... $\quad \mathbf{7 5}$

Plantation ....... 10

Loyer de la terre....... 200

Plantation de la haie vive ... 150

$1,970^{f}$

Les dépenses d'établissement seront donc, de $1,970 \mathrm{fr}$.

1 Les 600 pyramides donneront, de 10 ans à 14 ans, 40 fruits, en moyenne, soit pour 24,000 fruits à $150 \mathrm{fr} . \ldots \ldots \ldots . . . . . . .3,600$

De 15 à 40 ans, 80 fruits au moins, soit 48,000 à 150 fr. .............. 2,700 
ESPALIERS

Les espaliers coûtent beaucoup plus que les pyramides et les hautes tiges; mais ils produisent davantage. Cependant, nous ne conseillons pas cette culture comme spéculation à ceux qui n'auraient pas la vente des beaux fruits; elle ne leur produirait pas plus que la pyramide. Toutefois, l'espalier est l'un des plus beaux ornements d'un mur, et il n'est pas rare qu'il donne des fruits qui trouvent placement à 50 et 60 francs le cent.

Les fruits de l'espalier sont les plus beaux, les plus succulents et les plus assurés. Mais, pour bien conduire un espalier il faut une certaine science pratique que l'on rencontre rarement dans les campagnes. Il faut dono se borner à garnir ses murs et à travailler au mieux. Puis, sitôt qu'on aura acquis l'expérience nécessaire, on pourra agir plus en grand et faire des contre-espaliers, qui produisent également beaucoup et promptement.

CORDONS ET NAINS

On fait rarement des plantations de nains et de cordons pour la spéculation. Les nains s'emploient dans de mauvaises terres, et les cordons pour entourer les carrés. On fait également l'un et l'autre pour 
utiliser quelque coin de terre. Quoi qu'il en soit, les cordons donnent des fruits de belle et bonne qualité ; mais ces plantations ne sont pas de longue durée.

Quelques pieux en bois sulfaté, du fil de fer galvanisé, des raidisseurs, voilà à quoi se réduit la dóponse.

Une fois le sol défoncé, il faut le niveler. Siil est humide et qu'il y ait des eaux stagnantes à la surface, on ouvre des rigoles pour les amener à l'cxtrémité de la pièce ou dans un endroit où elles ne puissent nuire à la plantation.

Il est quelquefois utile de drainer; cette opération n'est pas très-coûteuse ; aussi ne faut-il pas la négliger quand le sol l'exige. Les arbres végéteront mieux et les fruits seront infiniment meilleurs.

Il est, dans ce cas, préférable d'employer des pierres, des gravats, des plâtras, des fagots, etc., au licu de tuyaux, parce qu'il arrive fréquemment que les racines des arbres pénètrent dans ces derniers et interceptent le passage à l'eau.

Une fois le sol bien nivelé, bien râtelé ou hersé, il faut tracer et ouvrir les trous.

On commence par mettre un jalon au bout de chaque ligne, puis on tend un cordeau et on mesure la distance. Ainsi, si l'on plante tous les 7 ou 8 mètres, on mesure cette distance et on plante un jalon 
à côté du cordeau, à l'endroit même où l'arbre doit se trouver. Quand la ligne est ainsi tracée, on ouvre des trous à la place de chaque jalon, en rejetant la terre à droite et à gauche de la ligne, pour pouvoir replacer le cordeau lors de la plantation. Les trous doivent être assez larges pour que les racines ne soient pas gênées pour entrer, et assez profonds pour que l'arbre soit enterré jusqu'au collet.

PLANTATION. - ÉpOQue a LA QৃUELLE ELL DOIT ÊTRE FAITE

La meilleure époque pour faire les plantations, est incontestablement l'automne, aussitôt que les pluies ont mouillé la terre, c'est-à-dire à partir du 10 novembre. On peut continuer de planter jusqu'en mars.

Si votre plantation doit durer quelques jours, mettez vos arbres en jauge, après avoir délié les paquets, et si la terre n'est pas assez humide, arrosez pour rendre de la fraîcheur aux racines. S'ils sont ridés et qu'ils semblent desséchés, jetez-les dans une mare ou dans l'eau et laissez-les y jusqu'à ce qu'ils aient repris leur apparence normale, puis plantez-les ou mettez-les en jauge.

Pour planter, on tend un cordeau d'un bout de la ligne à l'autre bout; on le fait passer, sur le milieu du trou, et, après avoir rafraîchi les racines, retranché les parties mutilées ou meurtries, supprimé.les branches cassées, on place l'arbre de manière que la 
greffe soit de $\check{\text { ou }} 6$ centimètres plus haute que le cordeau, sil a été greffé rèz terre; autrement on ne s'occupe que du collet. Cela fait, on remplit le trou en disposant les racines par étages, comme elles étaient avant l'arrachage; on secoue très-légèrement pour qu'elles ne soient pas dans le vide, et on presse doucement la terre autour pour l'appuyer, afin que les vents ne culbutent ou ne déplacent pas l'arbre. Il faut être deux : l'un le tient, et l'autre recouvre de terre les racines.

Si l'arbre a un pivot il faut le plier et le coucher au fond du trou pour l'arrêter et le transformer en racine horizontale ou traçante. On peut aussi le supprimer ; mais souvent il se reforme.

En agissant ainsi, on est sûr que la plantation est en ligne, par le pied du moins. Quand une rangée est terminée, on n'a plus qu'à redresser les tiges qui sont mal placées. Cela devient très-facile entre deux : l'un se place à une extrémité et aligne le tout, pendant que l'autre fait les redressements.

Plantez toujours un peu haut, parce que les terres étant fraîchement remuées, il se fera du tassement, et l'arbre s'enfonce toujours de plusieurs centimètres. Il faut que l’étage supérieur des racines ne soit pas à plus de 14 centimètres de profondeur.

Plantez un peu plus profond dans les sols secs et légers que dans ceux qui sont humides et compactes; mouillez au besoin les racines pour y faire adhérer la terre; arrosez pour la plornber; mettez un luteur s'il y a danger que l'arbre soit dérangé par 
les vents et paillez avec du fumier plutôt qu'avec de la litière. Cela maintiendra mieux la fraîcheur.

C'est, du 'reste, le collet qui doit guider le planteur; il faut qu'il soit à fleur du sol.

N'enterrez jamais la greffe; l'arbre s'affranchirait, et cela jetterait la perturbation dans la végétation et causerait la mort dans certaines circonstances.

Ne plantez jamais par la pluie ou par les gelées; vous vous exposeriez à perdre vos arbres.

Plantez les espaliers à 12 centimètres au moins des murs, et arrosez-les, au printemps, une fois par semaine, si la terre est sèche, jusqu'à la reprise. Passé cela, ne vous en occupez plus, si ce n'est en été, quand la chaleur et la sécheresse sont excessives. Il faut alors les arroser et les bassiner le soir, tous les quinze jours au besoin.

Au mois de mars, paillez vos arbres avec du fumier à demi pourri ou du fumier provenant de vieilles couches. Cette opération entretient la frâ̂cheur et la végétation : la reprise devient alors plus assurée.

Quelques amateurs poussent la précaution jusqu à cnduire d une couche de bouse de vache, délayée dans de l'eau, les tiges des arbres à plein vent un peu fortes ou qui sont rabotteuses ou meurtries. Cela fait également le plus grand bien aux espaliers. C'est une opération peu coûteuse et bientôt faite. On peut la renouveler plusieurs fois, si cela est nécessaire, pendant les premières années. 
F.IUT-IL TAILLER LES ARBRES LORS DE LA PLANTATION?

Quelques auteurs, et notamment des pépiniéristes, recommandent de ne faire aucune suppression aux branches des arbres lors de la plantation, afin, disent-ils, de permettre aux racines de se former.

Il est vrai qu'en taillant très-court un arbre, on lui enlève le plus puissant moyen de se faire non des racines, mais des spongioles, puisque, privé de feuilles il est privé de respiration.

- En ne taillant pas, on perd une année pour la formation de l'arbre, et c'est là un grand inconvénient. D'autre part, les yeux de la base, soit des scions d'un an, soit des rameaux destinés à former la charpente d'une haute tige, s'annulent, et souvent il est très-difficile d'obtenir des bourgeons bien placés : autre inconvénient plus grave encore, puisque souvent on est obligé de ravaler, l'année suivante, ce qui fait perdre deux ans au lieu d'un.

Si l'on veut bien se rendre compte de ce qui se passe après la plantation, on verra que la végétation commence aussitòt que les spongioles nouvelles se sont formées pour remplacer les racines supprimées à l'arrachage et les anciennes spongioles desséchées ou mutilées. Quand les spongioles fonctionnent, la circulation se rétablit. On peut alors et sans inconvénient supprimer la majeure partie des feuilles; car la sève, dans son mouvement ascensionnel, fait développer les yeux laissés par la taille, et il n’y a 
qu'un ralentissement de végétation, de courte durée. Donc, on peut tailler un arbre quand il a poussé quelques feuilles.

Nous avons essayé des deux modes d'opérer et nous avons trouvé celui-ci infiniment supérieur à celui de ne pas tailler. Nous croyons que si quelques pépiniéristes donnent le conseil de ne faire aucune suppression, c'est parce qu'ils livrent souvent de vieux arbres rebottés, usés en pépinière, et qui ont besoin de tous les secours de l'art pour ne pas succomber à la transplantation; car un arbre sain, pourvu de bonnes racines, ne saurait souffrir d'une taille faite dans les conditions que nous venons d'énoncer. Du reste, cette opinion est partagée par un grand nombre de praticiens distingués parmi lesquels nous citerons M. Hardy.

A QUelle distance IL faut planter? — age des arbres

Nous avons, dans un chapitre précédent, parlé de la distance moyenne à laquelle on peut planter; nous croyons devoir revenir sur cette quistion, parce qu'elle est importante et que nous ne l'avons traitée qu'incidemment.

On sait que plus le sol est riche, plus les plantes prennent de développement, que plus il est pauvre, plus elles sont faibles. C'est sur ce principe qu'est basé l'usage de semer dru dans les mauvaises terres, et clair dans les terres substantielles.

Si cela est vrai et utile pour les plantes annuelles, 
comme les céréales qui occupent le terrain pendant une année seulement, à plus forte raison pour les arbres qui vivent un demi-siècle et plus à la même place.

Ce principe posé et reconnu, il s'agit de le mettre en pratique.

Est-il possible, à première vue, de dire à quelle distance on doit planter les arbres dans un terrain quelconque? Non! on ne peut faire que des appréciations approximatives, car il y a des variétés qui prennent plus ou moins de développement : ainsi un beurré Clairgeau, un Passe-Colmar n'atteindront jamais la dimension d'un Triomphe de Jodoigne.

Cependant, nous croyons que les hautes tiges seront convenablement espacées comme il suit :

Sols riches, profonds et frais. $8^{\mathrm{m}}$ en tous sens.

Sols substantiels et profonds. $7^{\mathrm{m}}$

Sols moins profonds .... $6^{\mathrm{m}}$

Sols légers, assez fertiles et profonds........ $\check{5}^{\mathrm{m}}$

Sols légers, peu fertiles et peu profonds. ...... $4^{\mathrm{m}}$

Les pyramides seront bien espacéos comme il suit:

Sols riches, frais et profonds. $4^{\mathrm{m}}$ en tous sens

Sols substantiels et profonds. $3^{\mathrm{m}} 50^{\mathrm{c}}-$

Sols moins profonds . . . $3^{\mathrm{m}}$

Sols légers et profonds . . . $2^{\mathrm{m}} \mathrm{s0}^{\mathrm{c}}$

Sols légers, peu fertiles et peu

profonds...... $2^{\mathrm{m}}$ 
Si nous insistons sur les distances à observer entre les arbres, c'est parce qu'elles ont une grande importance pour le planteur. En effet, s'il plante trop dru dans les terres riches et substantielles, les arbres s'étiolent, s'élancent, produiisent peu de fruits et de mauvaise qualité. Sil plante trop espacé, c'est du terrain occupé en pure perte. Dans les mauvais sols, il en est de même; planter trop dru a le même inconvénient; trop espacé, le terrain est également improductif.

Ne plantez jamais un vieil arbre, ce serait de l'argent et du temps perdus. A cinq ou six ans, un arbre à haute tige est bon à mettre en place; il faut qu'il ait un an ou deux de greffe.

Les pyramides, espaliers, cordons, nains ne doivent avoir qu'une année de greffe. Avec des scions d'un an, on obtient tout ce qu'on veut; les rameaux sont pourvus de bons yeux, l'écorce est lisse, les racines sont nombreuses, le chevelu est beau, la reprise est certaine. Donc, pas de vieux arbres.

Certaines personnes croient jouir plus vite en plantant des arbres tout formés. C'est une erreur qui coûte fort cher. Si l'on obtient parfois des fruits à la seconde année de plantation, c'est au détriment de l'arbre qui n'en donne plus et qui meurt ou végète ensuite.

Transplanter de grands arbres est un tour de force qu'on ne recommence pas deux fois. Il est bien plus sage de se résigner à attendre quelques années. Si l'on veut jouir de suite, mieux vaut planter des 
arbres en touffes, les mutiler par les pincements pour avoir du fruit à l'âge de trois ans, et les arracher quand les autres sont en rapport.

FAUT-IL PLANTER DES POIRIERS GREFFÉS SUR FRANC OU SUR COIGNASSIER ?

Il y a des terres qui veulent le coignassier, d'autres le franc : c'est donc au planteur à bien connaître son sol. Il y a aussi des variétés qui doivent toujours être greffées sur franc pour réussir.

Pour les sols secs, légers et presque arides, ceux qui sont brûlants, fortement calcaires, pierreux, mais profonds, il faut choisir des poiriers greffés sur franc. La profondeur du sol est une condition essentielle; car, dans les terres dont le sous-sol est imperméable aux racines, au pivot, ils réussissent mal.

Pour les sols frais, substantiels et fertiles, qu'ils soient argilo-calcaires ou siliceux; dans les terres franches ou dans les sables gras, dans les alluvions même un peu compactes, on peut planter des arbres greffés sur coignassier.

Le poirier greffé sur franc est plus vigoureux, il atteint une plus grande taille et vit plus longtemps. Il n'est pas rare de voir des arbres ainsi greffés qui ont un siècle et demi et plus.

Greffé sur coignasșier, il fructifie plus vite, ses fruits sont plus savoureux, plus abondants; mais l'arbre s'épuise rapidement; il reste d'une taille médiocre et vit moins longtemps. Il est rare de voir des 
poiriers sur coignassier de l'âge de soixante ans.

Si toutes les variétés de poires réussissent sur franc, il n'en est pas de même de celles greffées sur coignassier. Il y en a qui n'admettent pas ce sujet; telles sont : la bergamotte silvange, le beurré clairgeau, la bergamotte d'Angleterre, le beurré Napoléon, la bonne de Malines, le beurré gris d'hiver, le bon chrétien de Rans, délices de Louvenjoul, beurré d'Apremont, bergamotte fortunée, Van Mons de Léon Leclerc, colmar d'Aremberg, etc. Cependant, pour faire des pyramides, des cordons, des espaliers, on peut les employer greffées sur coignassier dans les suls qui ont suffisamment de fraîcheur: ce sera le moyen de jouir plus vite et d'avoir de plus beaux et de meilleurs fruits.

Quelques arboriculteurs plantent des sauvageons et les greffent sur place après reprise. Cette méthode peut réussir dans les sols très-profonds, mais elle est très-vicieuse dans ceux qui ne le sont pas; parce que les arbres pivotent, ne se font ni racines traçantes ni chevelu, et quand ils arrivent sur le sol infertile, ils ne font plus que végéter.

DOIT-ON PLANTER DES ARBRES A PLEIN TENT OU DES PXRAMIDES

Cette question n'intéresse guère que la grande production; car dans les jardins bourgeois, chez le propriétaire où il ne s'agit que de produire des fruits pour la maison, elle est bien vite résolue. Le bourgeois qui a du temps à lui pour soigner ses arbres, 
CULTURE DES ARBRES FRUI'TIERS

ou un jardinier, et qui tient à avoir un jardin coquet, préférera l'espalier ou la pyramide; tandis que le cultivateur, qui ne dispose que rarement de quelques instants, préférera un verger, parce qu'il ne réclame q ue quclques soins en hiver, époque à laquelle il pe ut les lui donner.

Les arbres de plein vent sont longtemps sans fructifier, surtout quand ils sont plantés dans un sol riche; ils sont tellement vigoureux qu'ils ne produisent que du bois pendant les sept ou huit premières années de plantation.

Les pyramides produisent à l'âge de 4 ou 弓̆ ans.

Les arbres à haute tige n'exigent pas ou peu de taille; par conséquent peu de temps et de savoir.

Les pyramides demandent, pendant les six premières années, des soins continuels; beaucoup de temps pour la taille et les pincements, de l'habitude et du savoir-faire pour équilibrer la sève et la bien répartir dans toutes les parties de l'arbre.

La culture des arbres à haute tige permet d'occup»r le terrain où ils sont plantés pendant une dizaine d'années, celle des pyramides l'interdit en grande partie.

Les arbres à haute tige veulent un sol un peu plus riche et plus profond que les pyramides.

Les arbres à haute tige vivent et produisent longtemps.

Les pyramides ont une durée beaucoup moins longue.

Tols sont les inconvénients et les avantages des deux systèmes; c'est au planteur à interroger ses 
besoins, son terrain, et à résoudre la question. Le jeune homme qui plantera des pyramides pourra les voir s'éteindre, tandis que ses arrière-petits-fils verront encore les arbres à plein vent.

LISTE dES 30 variétés de poIRIERS Les pLUS Recommandables POUR LA GULTURE A PLEIN VENT

Faire une plantation d'arbres fruitiers sans se rendre un compte exact de la valeur des variétés, c'est agir en aveugle, s'exposer à n'avoir ou que de mauvais fruits ou des fruits mûrissant tous à la même époque. Rien n'est donc plus important que de faire un bon choix, afin d'avoir, comme amateur, des fruits de première qualité se conservant longtemps, et comme spéculateur de faire le plus d'argent possible. Que le spéculateur sache surtout que plus les fruits sont de longue garde, plusils ont de valeur. Ainsi, on vend à Paris 30 à 40 centimes une poire de Duchesse qui ne passe pas le mois de novembre; si on pouvait en avoir au mois de mai, comme on a plusieurs autres variétés, on les vendrait faci lement 1 franc et plus.

Il y a un grand nombre de variétés de poiriers ; on les compte par centaines; mais il $y$ en a à peine 40 ou 50 qui doivent être particulièrement cultivées. Celles que nous allons désigner sont généralement les meilleures, les plus fertiles, les plus belles et les plus vigoureuses. Nous disons généralement, car la qualité de certaines poires varie un peu suivant les 
latitudes et le sol. Ainsi le Williams est parfois trèsmusqué; mais ce sont là des exceptions fort rares que le planteur doit apprécier et éviter.

Voici celles qui sont le plus propres à la culture en plein vent, par ordre de maturité et de mérite.

Seulement, nous ferons remarquer que l'époque de la maturité peut varier suivant le climat, la nature du sol et de la température, et qu'il n'est pas rare d'avoir un écart de 15 jours.

\section{Juillet}

Beurré Giffard;

Épargne (beau présent, Saint-Samson, cuisse madame, cueillette) ;

Doyenné de juillet (roi Jolimont) ${ }^{1}$.

Août

Beurré Goubault ;

Duchesse de Berry d'été;

Rousselet d'août.

Septembre

Louise Bonne d'Avranches (Louise de Jersey, Bonne de Longueval);

Bonne d'Ézée;

Beurré d'Angleterre ${ }^{2}$.

1. Ce fruit ne se recommande que par sa précocité.

2. Nous lui préférons de beaucoup la précoce verte longue de la Sarlhe; mais ce fruit se transporte moins bien, en raison de l'abondance de son eau et de sa chair très-fondante. Pour le verger bourgeois, c'est l'un des meilleurs fruits. Il est au moins égal à la Louise Bonne d'Avranches. 


\section{Octobre}

Seigneur (Bergamotte lucrative, bergamotte fiévée, fondante d'automne);

Frédéric de Wurtemberg (médaille d'or);

Saint-Michel archange.

\section{Novembre}

Triomphe de Jodoigne;

Nec plus Meuris (beurré d'Anjou);

Épine du Mas (duc de Bordeaux, Colmar-du-Lot).

\section{Décembre}

Joséphine de Malines;

Bonne de Malines (colmar Nélis, Nélis d'hiver);

Beurré Millet.

\section{Janvier à mai.}

Bergamotte Espéren;

Bergamotte fortunée;

Doyenné d'Alençon ;

Passe-Colmar ;

Vauquelin ;

Suzette de Bavay;

Beurré Bennert;

Saint-Herblain d'hiver;

Poire Devergnies;

Docteur Bouvier;

Franceline Millot ;

Martin sec. 
Nous devons faire remarquer que, comme nous l'avons dit en tête de ce chapitre, les variétés sont par ordre de mérite : ainsi, si l'on voulait choisir les sept meilleures, soit une de chaque saison, il faudrait choisir le beurré Giffard, beurré Goubault, Louise bonne d'Avranches, Seigneur, Triomphe de Jodoigne, Joséphine de Malines, Bergamotte espéren (celle-ci comme garde). Martin sec est une poire à cuire, hors de série.

Remarque. - Docteur Bouvier d'hiver, beurré Bennert, Franceline Millot sont des variétés très-rares qui ne se rencontrent presque pas dans le commerce.

Liste des 30 variétés les plus méritaNtes pour espaliers PYRAMIDES, TOUFFES ET GORDONS

PAR ORDRE DE MATURITÉ ET DE QUALITÉ

Tout ce que nous avons dit des variétés propres au plein vent s'applique à celles-ci. Voici les trente plus méritantes pour pyramides, espaliers, cordons, etc.

\section{Poires mûrissant en Juillet}

Beurré Giffard ;

Épargne (beau présent, Saint-Samson, cuisse madame, cuẹillette);

Doyenné de juillet (roi Jolimont) ${ }^{1}$.

1. Comme nous l'avons dit plus haut, ce fruit n'a d'autre mérite que sa précocité. 


\section{Août-Septembre}

Bon chrétien Williams (Barnet's, bon chrétien Barnet's, Williams Pear, Bartelet de Boston);

Beurré Goubault;

Duchesse de Berry d'été.

\section{Septembre}

Louise bonné d'Avranches (Louise de Jersey, bonne de Longueval) ;

Bonne d'Ézée;

Beurré d'Amanlis'.

Octobre

Duchesse d'Angoulême (duchesse);

Seigneur (fondante d'automne, bergamotte fiévée, bergamotte lucrative);

Beurré d'Apremont (Beurré Bosc).

Novembre

Beurré Clairgeau ;

Colmar d'Aremberg ;

Délices de Louvenjoul.

Décembre

Beurré Diel (beurré magnifique, beurré incomp: a ble, beurré royal, beurré des Trois tours);

1. Nous lui préférons la précoce verte longue de la Sarthe, seulement clle se transporte et se conserve moins bien (voir la note à la liste des 30 variétés à haute tige). 
Triomphe de Jodoigne ;

Beurré de Luçon (beurré gris d'hiver nouveau). 4

\section{Janvier}

Passe-Colmar (passe-colmar gris ou doré) ;

Doyenné d'Alençon (doyenné d'hiver nouveau) ;

Joséphine de Malines,

Beurré d'Hardenpont (beurré d'Aremberg, GlouMorceau).

\section{Février jusqu'en mai}

Bergamotte espéren ;

Bergamotte fortunée;

Beurré de Nivelles ;

Saint-Herblain d'hiver ;

Suzette de Bavay ;

Commissaire Delmotte;

Catillac (poire à cuire);

Martin sec ou rousselet d'hiver (poire à cuire).

Ainsi que nous l'avons déjà fait remarquer, la première variété en tête de chaque saison est préférable aux autres généralement. Quant aux deux dernières variétés mûrissant de fóvrier en mai, ce sont des poires à compote.

L'amateur fera bien de s'en tenir à ces trente variétés et de n'essayer des autres qu'en petit, celles-ci sont d'un produit certain et d'une qualité exceptionnelle.

Remarque. - On fera bien d'étudier la tardive de 
Toulouse ou duchesse d'hiver, pour la mettre en parallèle avec Commissaire Delmotte, Beurré de Nivelles et Saint-Herblain d'hiver.

En lisant nos listes, la plupart des personnes qui se sont occupées d'arboriculture se demanderont pourquoi nous avons exclu un grand nombre de vieilles variétés très-recommandables anciennement, telles que le Saint-Germain, le Bézi Chaumontel, le doyenné d'hiver, le bon chrétien d'hiver, crassane, doyenné blanc, etc.

Ces arbres sont usés et ne réussissent plus que dans certaines localités, dans des terres et à des expositions privilégiées. Du reste, les grandes formes ne leur conviennent plus; la haute tige est impossible pour la plupart, et il faut avoir recours à l'espalier pour en tirer quelques fruits qui, tantôt passables, tantôt médiocres, sont le plus souvent tavelés et sans qualité.

Il ne faut donc plus compter sur ces variétés pour avoir de beaux fruits, ils sont usés comme les arbres qui les produisent et on les rejetterait s'ils n'avaient pas pour eux leur ancienne réputation. Que de gens ne vantent les choses que parce qu'ils les ont entendu vanter. L'amateur peut essayer encore un espalier de chaque, par curiosité ; mais le spéculateur doit les considérer comme éteintes et n'existant plus. 
QUANTITE DE GHAQUE VARIÉTE A PLANTER

r uisqu'il est indispensable d'avoir des fruits le plus ongtemps possible pour la consommation et pour la vente, ceux d'hiver sont donc les plus précieux; mais comme il est utile d'en avoir constamment à sa disposition, on doit combiner les plantations de manière à en manquer le moins longtemps possible. Ainsi, si l'on a de l'espace pour placer 100 arbres, on devra planter en plein vent :

2 Beurré Giffard,

2 Épargne,

2 Doyenné de juillet,

2 Beurré Goubault,

2 Duchesse de Berry d'été,

2 Rousselet d'août,

3 Louise bonne,

2 Bonne d'Ézée,

2 Beurré d'Angleterre ou 2 Précoce verte longue de la Sarthe,

4 Seigneur,

3 Frédéric,

4 Saint-Michel archange,

fj Triomphe de Joidoigne,

A reporter. 35 
Report. 35

3 Nec plus Meuris,

4 Épine Du Mas,

3 Joséphine de Malines,

4 Bonne de Malines,

ら Beurré Millet.

๖ Bergamotte Espéren,

5 Bergamotte fortunée,

ら Doyenné d'Alençon,

丂 Passe-Colmar,

4. Vauquelin,

ऽ Suzette de Bavay,

4 Beurré Bennert,

4 Saint-Herblain d'hiver,

3 Poire Devergnies,

2 Poire docteur Bouvier,

2 Franceline Millot,

2 Martin sec.

100

Si l'on n'a de place que pour 50 haute tige, il faut modifier la liste comme il suit :

2 Beurré Giffard,

1 Épargne,

1 Doyenné de juillet,

2 Beurré Goubault,

1 Duchesse d'été,

2 Louise Bonne,

1 Bonne d'Ézée, 
Report. 10

2 Beurré d'Angleterre ou 2 Précoce verte longue de la Sarthe,

2 Seigneur,

2 Saint-Michel archange,

3 Triomphe de Jodoigne,

2 Épine Du Mas,

2 Joséphine de Malines,

2 Bonne de Malines,

2 Beurré Millet,

3 Bergamotte Espéren,

3 Bergamotte fortunée,

2 Doyenné d'Alençon,

3 Passe-Colmar,

2 Vauquelin,

2 Suzette de Bavay,

2 Beurré Bennert,

2 Poire Devergnies,

2 Franceline Millot,

2 Martin-sec.

50

Pour 25, il faudrait faire le choix suivant :

1 Beurré Giffard,

1 Épargne,

1 Beurré Goubault,

1 Duchesse d'été,

2 Louise Bonne,

I Bonne l'Ézée,

A reporter. 7 
Report. 7

1 Seigneur,

1 Saint-Michel archange,

2 Triomphe de Jodoigne,

1 Épine Du Mas,

2 Joséphine de Malines

1 Bonne de Malines,

2 Bergamotte Espéren,

2 Bergamotte fortunée,

1 Doyenné d'Alençon,

2 Passe-Colmar, -

2 Suzette de Bavay,

1 Martin sec.

25

Si l'on n'a place que pour 12 arbres, il faut se borner aux suivants :

1 Beurré Giffard,

1 Beurré Goubault,

1 Louise Bonne,

1 Seigneur,

1 Saint-Michel archange,

1 Triomphe de Jodoigne,

1 Joséphine de Malines,

1 Bergamotte Espéren,

1 Bergamotte fortunée,

1 Doyenné d'Alençon,

1 Passe-Colmar,

1 Suzette de Bavay. 
Enfin, si l'on n'en peut placer que 6 , il convient de s'arrêter à ceux-ci :
1 Beurré Giffard,
1 Louise Bonne,
1 Seigneur,
1 Triomphe de Jodoigne,
1 Joséphine de Malines,
1 Bergamotte Espéren.
6

En suivant ces indications, on aura des fruits à consommer ou à vendre depuis le mois de juillet jusqu'à la fin d'avril.

Quant aux pyramides, espaliers, etc., voici comment la répartition devra se faire.

Pour 100 pyramides, espaliers, touffes ou cordons, choisissez :

2 Beurré Giffard,

2 Épargne,

2 Doyenné de juillet,

2 Bon-chrétien Williams,

2 Beurré Goubault,

2 Duchesse de Berry d'été,

4 Louise Bonne,

2 Bonne d'Ézée,

3 Beurré d'Amanlis ou 3 Précoce vert? longue de la Sarthe,

3 Duchesse d'Angoulême, 
Report. 24.

4 Seigneur,

3 Beurré d'Apremont,

4 Beurré Clairgeau,

3 Colmar d'Aremberg,

3 Délices de Louvenjoul,

๖ Triomphe de Jodoigne,

4 Beurré de Luçon,

כ Passe-Colmar,

こ Doyenné d'Alençon,

๖.Joséphine de Malines,

4 Bonne de Malines,

丂 Beurré d'Hardenpont,

こ Bergamotte Espéren,

5 Bergamotte fortunée,

4 Beurré de Nivelles,

3 Saint-Herblain d'hiver,

5 Suzette de Bavay,

2 Commissaire Delmotte,

1 Catillac,

1 Martin sec.

\section{0}

Pour こ0, les nombres suivants seront convenables :

1 Beurré Giffard,

1 Épargne,

1 Bon-chrétien Williams,

1 Beurré Goubault,

2 Louise Bonne,

A reporier. 6 
Report. 6

1 Bonne d'Ézée,

2 Beurré d'Amanlis ou 2 Précoce verte longue de la Sarthe.

2 Duchesse d'Angoulême,

2 Seigneur,

2 Beurré d'Apremont,

2 Beurré Clairgeau,

2 Colmar d'Aremberg,

2 Beurré Diel,

3 Triomphe de Jodoigne,

3 Beurré de Luçon,

3 Passe-Colmar,

3 Doyenné d'Alençon,

3 Joséphine de Malines,

3 Beurré d'Hardenpont,

3 Bergamotte Espéren,

3 Bergamotte fortunée,

3 Suzette de Bavay,

1 Catillac,

1 Martin sec.

50

Si $25 \%$ suffisaient pour employer le terrain que vous a vez de disponible, vous pourriez choisir :

1 Beurré Giffard,

1 Bon-chrétien Williams,

2 Bonne Louise,

1 Beurré d'Amanlis,

A reporter. $\tilde{5}$ 
Report.

2 Duchesse d'Angoulême,

1 Seigneur,

2 Beurré Clairgeau,

1 Colmar d'Aremberg,

1 Beurré Diel,

1 Triomphe de Jodoigne,

2 Passe-Colmar,

1 Doyenné d'Alençon, .

2 Joséphine de Malines,

2 Bergamotte Espéren,

2 Bergamotte fortunée,

2 Suzette de Bavay,

1 Martin sec.

25

Pour 12 variétés, le choix suivant serait bon :

1 Beurré Giffard;

1 Bon-chrétien Williams,

1 Bonne Louise,

1 Duchesse,

1 Beurré Clairgeau,

1 Beurré Diel,

1 Triomphe de Jodoigne,

1 Passe-Colmar,

1 Joséphine de Malines,

1 Bergamotte Espéren,

1 Bergamotte fortunée,

1 Suzette de Bavay. 
S'il n'y avait place que pour 6 , il conviendrait d'adopter les variétés suivantes :

1 Louise Bonne,

1 Duchesse d'Angoulême,

1 Triomphe de Jodoigne,

1 Passe-Colmar,

1 Bergamotte Espéren,

1 Bergamotte fortunée.

6

DESGRIPTION DES MEILEERS FRUITS

Avant d'entrer dans les détails de cette description, nous rappelons au lecteur que nous avons distingué, dans deux chapitres précédents, les variétés à cultiver en plein vent d'avec celles à mettre en espalier, pyramide, etc. Pour ne pas nous répéter et éviter de faire des classements fatigants, nous groupons ensemble les variétés des deux séries, d'autant plus qu'il y en a plusieurs qui figurent à la fois dans les arbres à haute tige et dans les pyramides, espaliers, etc.

Les silhouettes que nous donnons représentent les fruits de grosseur naturelle mais moyenne; plus souvent inférieure que supérieure. Quant à la forme, elle est la plus commune; cependant il y a parfois des écarts provenant du sol ou de la température.

Les 30 variétés propres au plein vent et les trento pour espalier et pyramides, confondues ensemble, 
ne représentent que 43 variétés différentes. Les voici par ordre alphabétique.

Nos 1. Beurré d'Amanlis,

2. - d'Angleterre,

3. - d'Apremont,

4. - Bennert,

5. - Clairgeau,

6. - Diel,

7. - Goubault,

8. - Giffard,

9. - d'Hardenpont,

10. - de Luçon,

11. - Millet,

12. - de Nivelles,

13. Bon-chrétien Williams,

14. Bonne d'Ézée,

15. Bonne de Malines,

16. Bergamotte Espéren,

17. Bergamotte fortunée,

18. Catillac,

19. Colmar d'Aremberg,

20. Commissaire Delmotte,

21. Délices de Louvenjoul,

22. Devergnies,

23. Docteur Bouvier,

24. Doyenné d'Alençon,

25. Doyenné de juillet,

26. Duchesse d'Angoulême,

27. Duchesse de Berry d'été,

28. Épargne, 
29. Épine Du Mas,

30. Franceline Millot,

31. Frédéric de Wurtemberg,

32. Joséphine de Malines,

33. Louise Bonne,

34. Martin sec.

35. Nec plus Meuris,

36. Passe-Colmar,

37. Rousselet d'aout,

38. Saint-Herblain d'hiver,

39. Saint-Michel archange,

40. Seigneur,

41. Suzette de Bavay,

42. Triomphe de Jodoigne,

43. Vauquelin.

NotA. - Les noms placés entre parenthèse sont les synonymes sous lesquels la poire est connue dans certaines localités. 


$$
\text { No } 1
$$

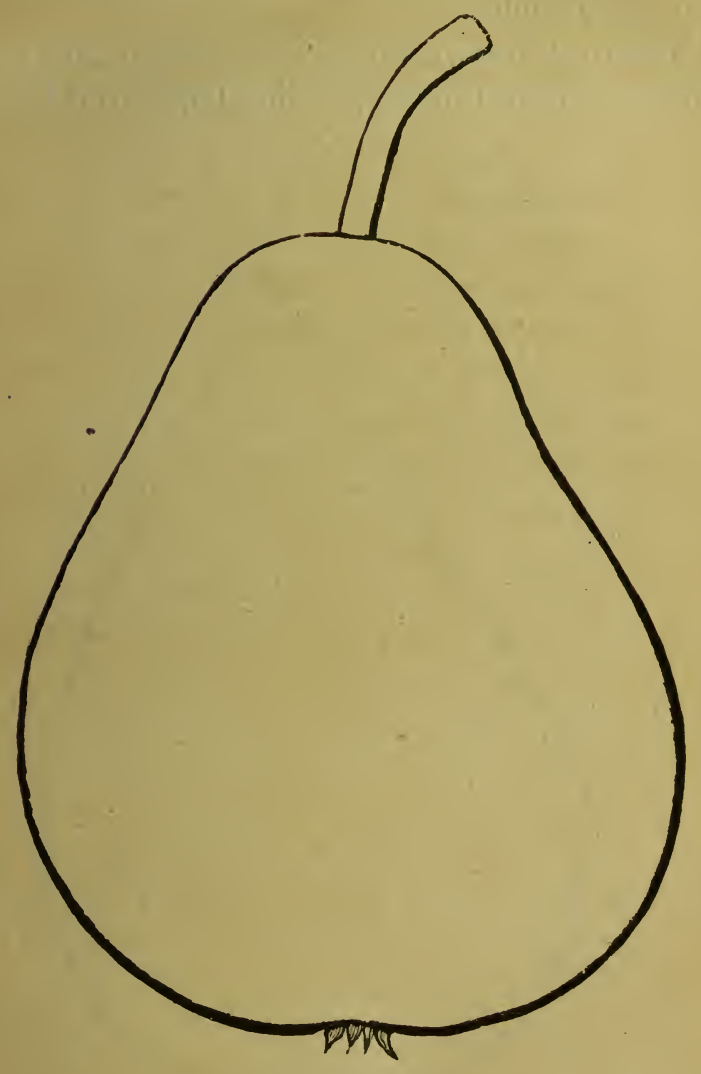

Beurró d'Amanlis (Wilhelmine, d'Albert). ¿

Fruit gros, ventru, peau épaisse, vert clair, nuan- 
cée de points roux, fouettée de rose du côté du soleil. Chair mi-fine, fondante, eau abondante, parfumée et un peu acidulée.

L'arbre est très-vigoureux; le bois est gros, les branches sont divariquées, feuilles larges, vert foncé. 
No 2

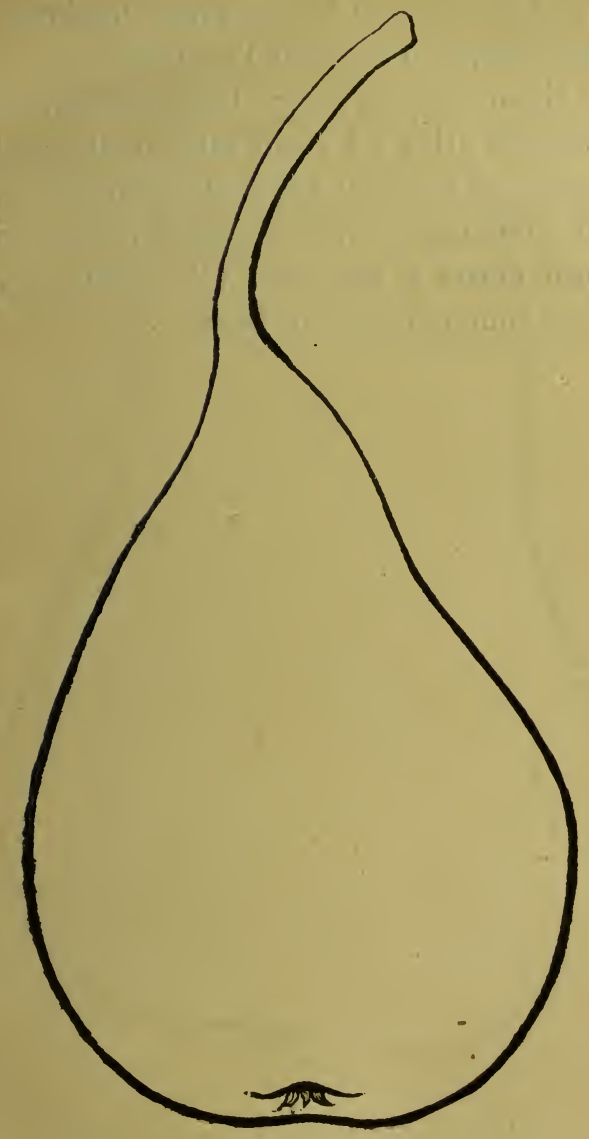

Beurré d'Angleterre.

Fruit moyen, pyriforme, peau vert clair, couverte 
de nombreuses taches de rouille peu foncées, disparaissant à l'époque de la maturité. Chair légèrement ambrée ou jaunâtre, mi-fine, fondante, eau abondante, sucrée, rappelant l'amande.

Arbre vigoureux sur franc, très-productif ; faible sur coignassier. Il y en a qui atteignent une taille gigantesque. Ne convient guère que pour les vergers. Nous lui préférons la verte-longue précoce de la Sarthe qui mûrit à peu près à la même époque; mais elle se transporte moins bien. 
$3 \mathrm{~N}^{\circ}$

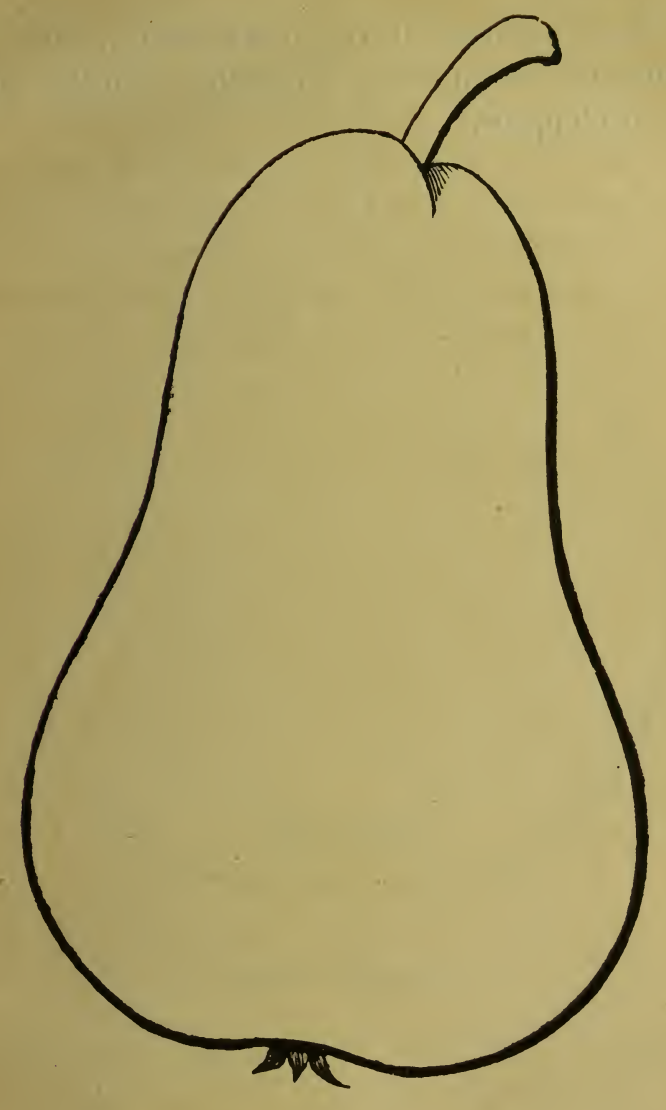

Beurró d'Apremont (Beurré Bosc).

Fruit assez gros, calebassiforme, peau presque 
rousse, chair très-fine, fondante, juteuse, d'une saveur exquise.

Arbre passablement vigoureux, fertile; faible sur coignassier. Jeune bois rougeâtre, feuilles larges très-développées. 


\section{$\mathrm{N}^{\circ} 4$}

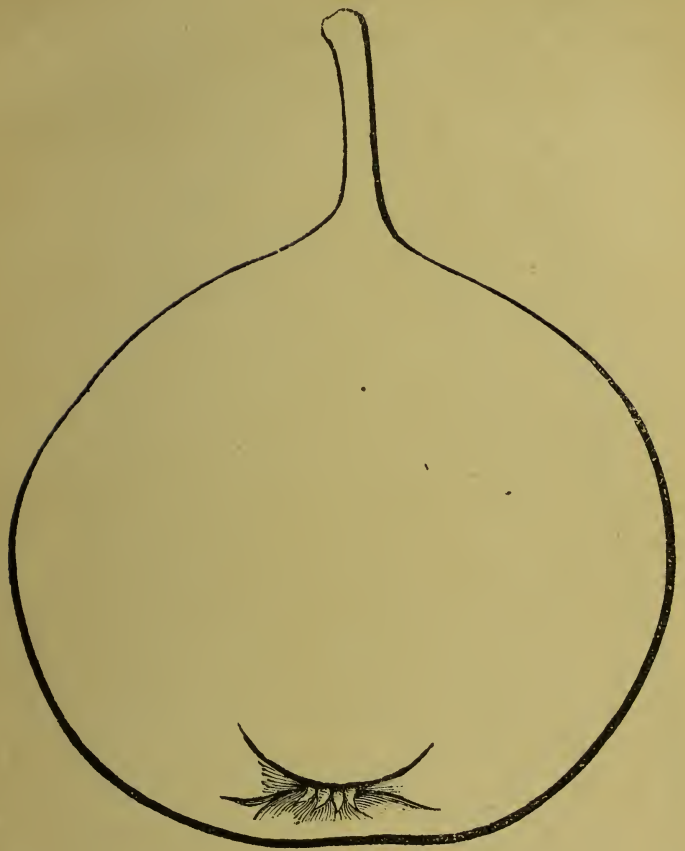

\section{Benrré Bennert.}

Fruit petit, turbiné. Peau lisse, jaune, colorée en brun rouge du côté du soleil, tiquetée de roux. Chair fondante, fine, beurrée, juteuse, sucrée et parfumée.

Arbre en forme de pyramide, vigoureux et fertile. Mieux sur coignassier que sur franc. 



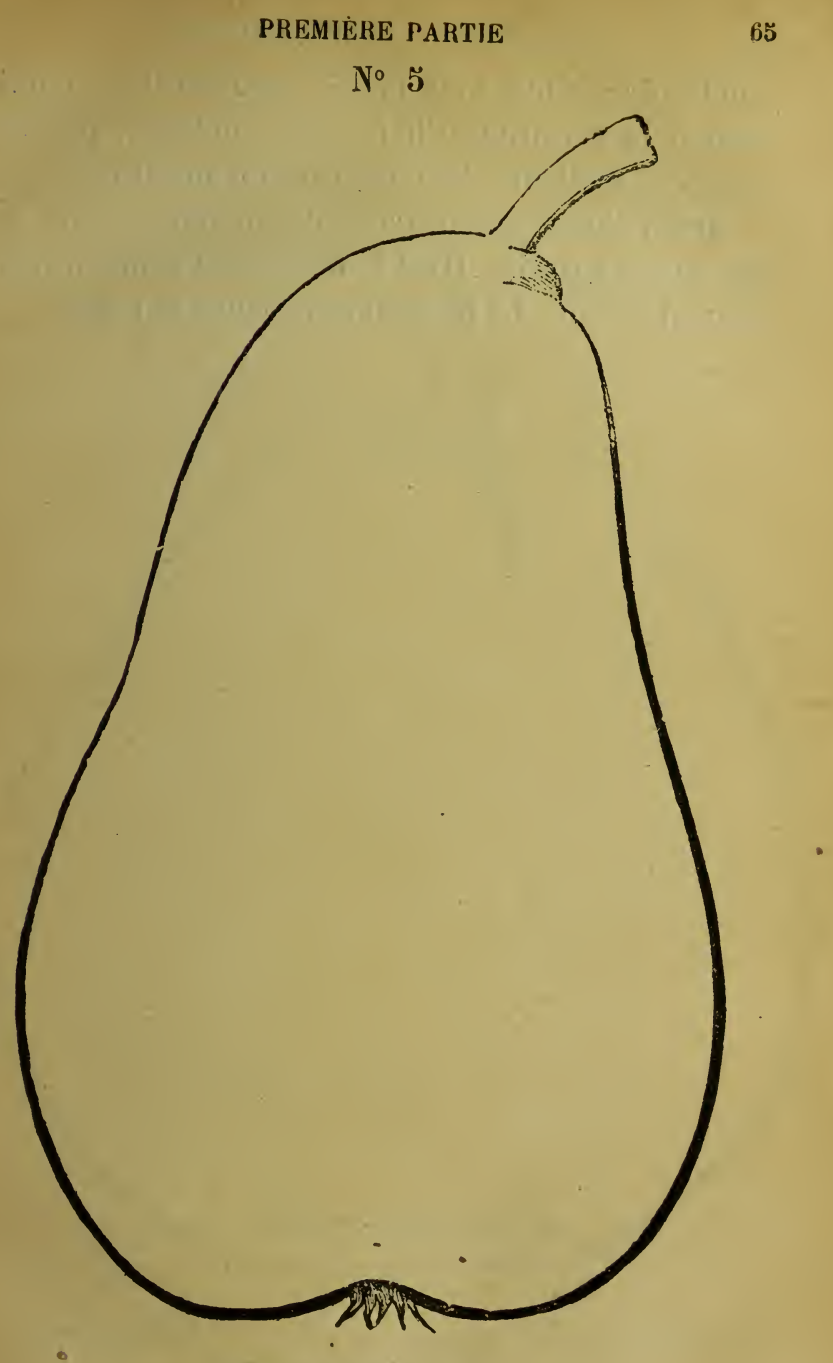

Beurré Clairgeau.

Fruit gros, calebassiforme, voûté; peau fine, ti4. 
quetée de points roux fauve, rougissant et jaunis sant à la maturité. Chair fine, fondante, juteuse, sucrée, excellente. Ce beau fruit ne mollit pas. Arbre d'une vigueur passable sur franc, mais faible sur coignassier. Il est très-fertile et se met promptement à fruit. Le bois est gros, court et rouge. 


\section{No 6}

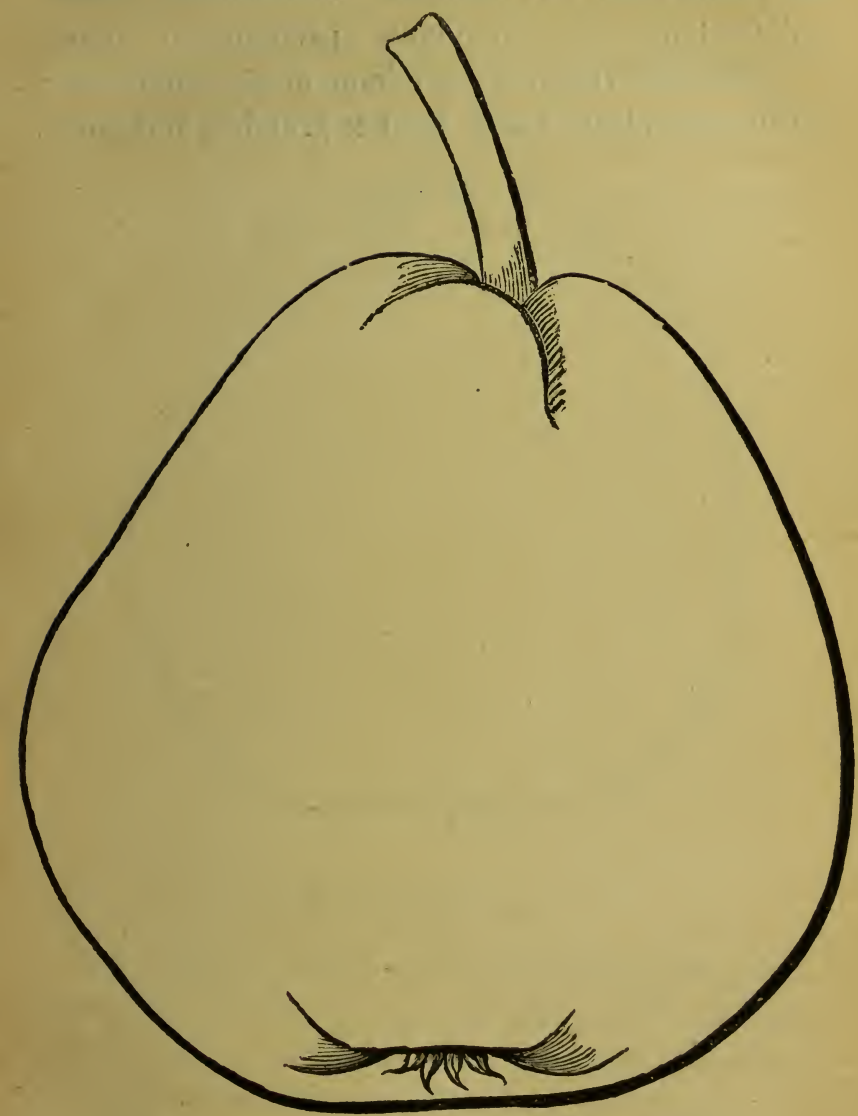

Beuré Dlel (Syn.: Beurré magnifique, Beurré incomparable, Beurré royal, Beurré des Trois Tours).

Fruit très-gros, turbiné, tronqué et ventru, peau 
verte avec quelques taches de rouille, mais passant au jaune foncé à la maturité. Chair mi-fine, ferme, mi-fondante, eau abondante, parfumée et sucrée. Arbre très-vigoureux sur franc et sur coignassier, fertile, bois brun foncé, feuilles grandes, vert foncé. 


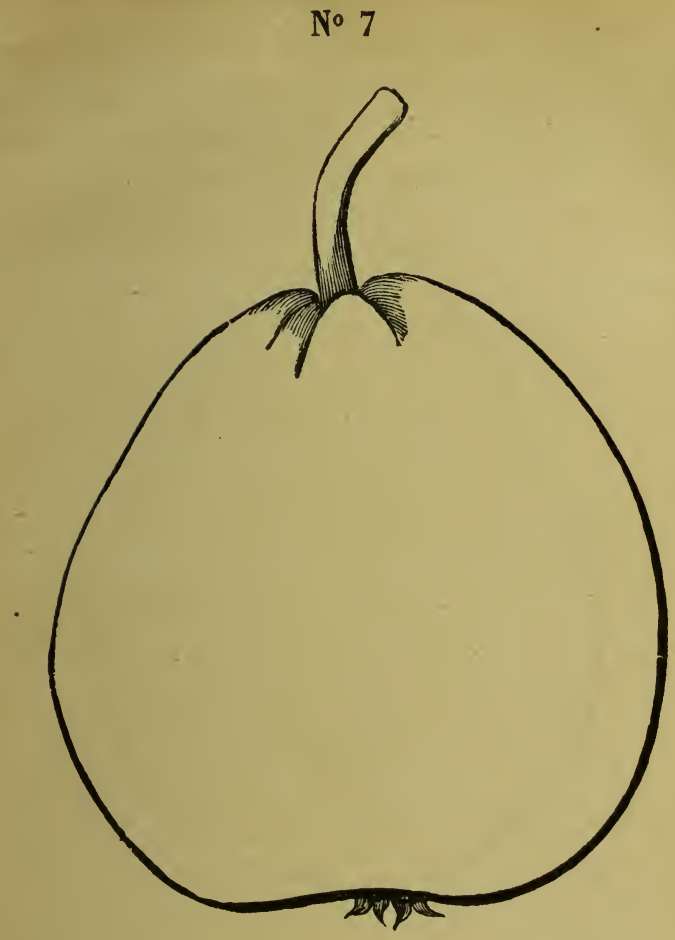

Beurré Coubault.

Fruit moyen, ayant un peu la forme du doyenné. Peau lisse, vert clair à la maturité, avec quelques points roux - chair blanche, fine, fondante, beurrée, juteuse, sucrée et agréablement parfumée.

Arbre vigoureux sur franc, généralement trop fertile pour être greffé sur coignassier; fait de jolies pyramides. 
<smiles>CC1=CCCCCCCCCCCCCCCCCC1</smiles> 


\section{№ 8}

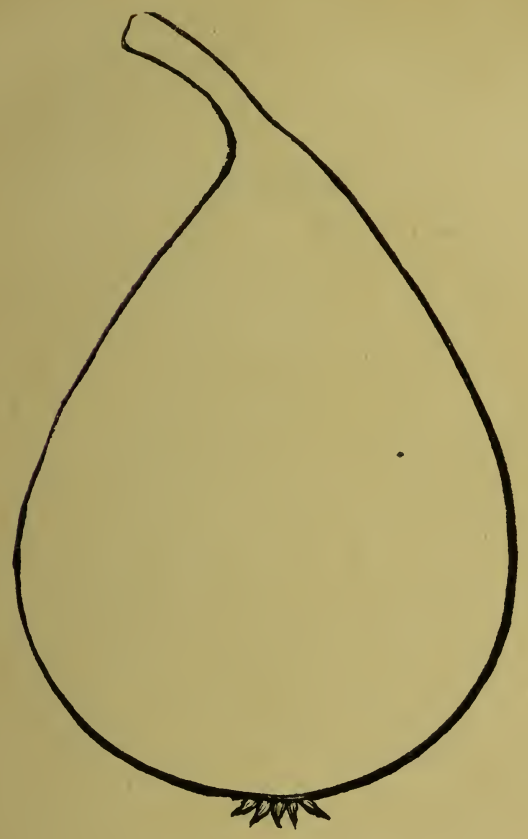

Beurré Gifard.

Fruit moyen, assez régulier, pyriforme, allongé, légèrement courbé au sommet; peau fine, vert clair, jaunissant à la maturité. Le côté du soleil est souvent pointillé de brun et de roux. Chair blanche, fine, fondante, juteuse, sucrée et légèrement parfumée.

Arbre assez vigoureux, à rameaux grêles, violets, avec des taches blanches; très-fertile. 


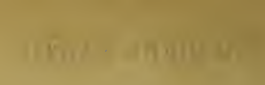


No 9

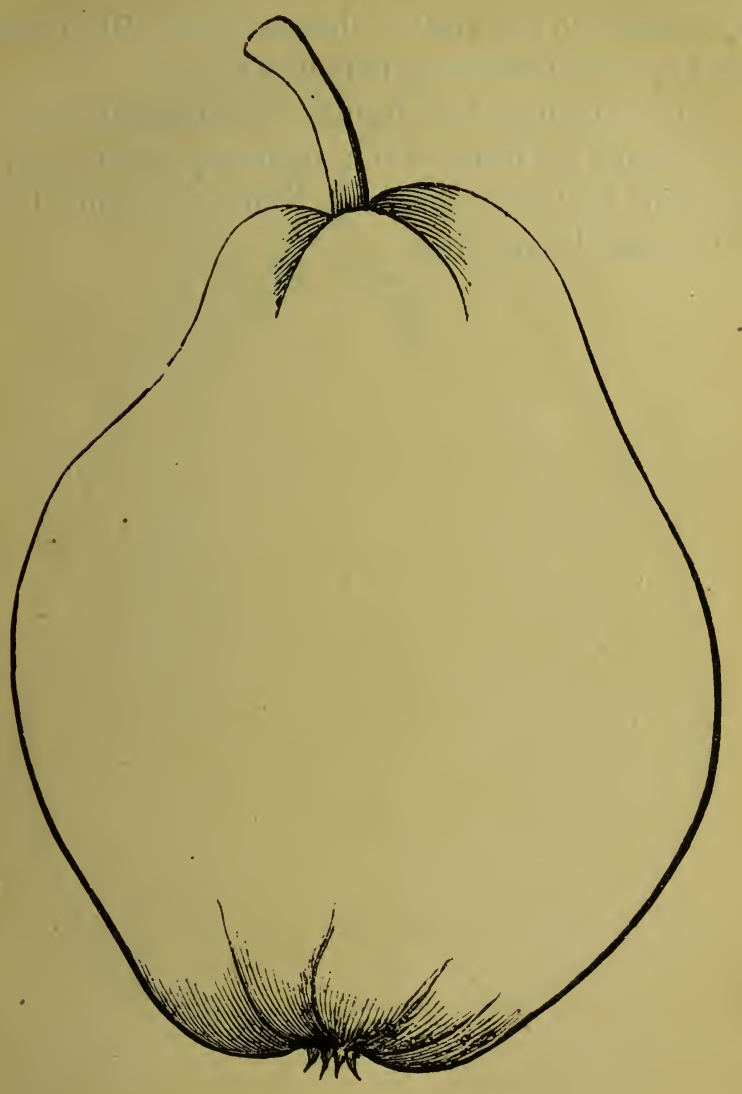

Beurré d'llardenpont (Beurré d'Aremberg en France, Glou-Morceau).

Fruit gros, côtelé, ventru, ressemblant au coing 
de Portugal ; peau vert clair, grasse et lisse, passant au jaune à la maturité, très-fine, très-sucrée, beurrée, fondante; ce fruit de bonne garde est l'un des plus recommandables que nous ayons.

Arbre vigoureux et fertile, se ramifiant bien et réussissant sur franc et sur coignassier. Il ne réussit pas dans les terres siliceuses, sèches; ses fruits tombent avant la maturité. 


\section{No 10}

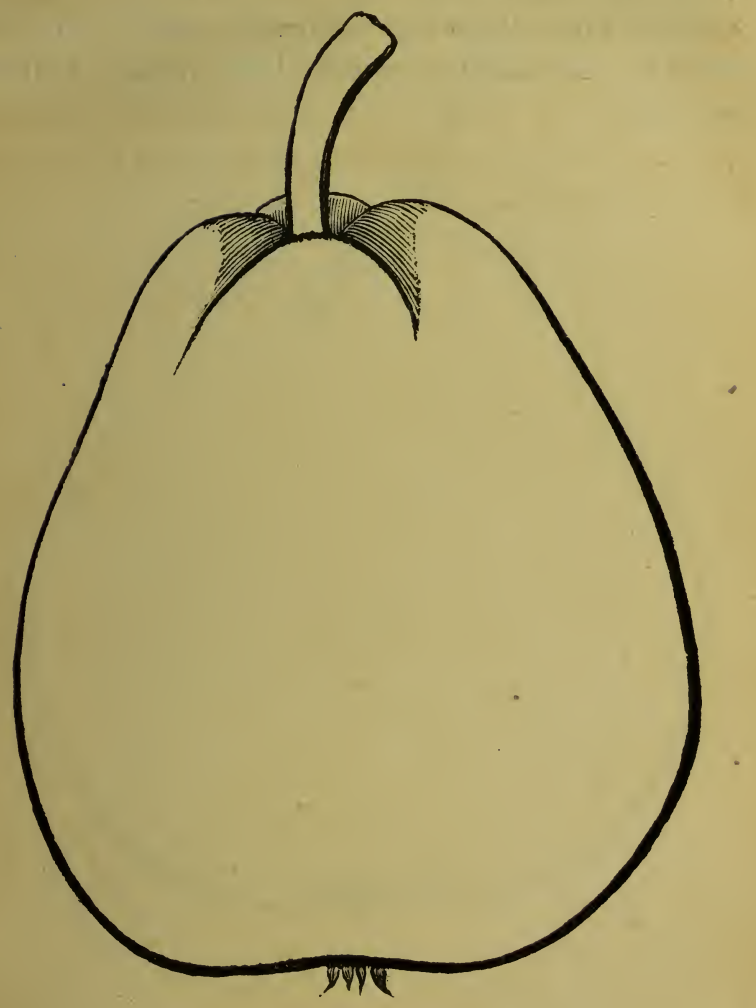

Beurré de Luģon (Beurré gris d'hiver nouveau).

Fruit gros, ovale, légèrement bossué, irrégulier; peau vert bronzé, tiquetée de taches de roux et do 
gris, parfois rouge du côté du soleil. Chair fine, mi. fondante, un peu graveleuse au centre, eau assez abondante et parfumée.

Arbre d'une vigueur moyenne; mal sur coignassier; très-fertile, jeune bois rouge; feuilles vert clair. 


\section{$\mathrm{N}^{\circ} 11$}

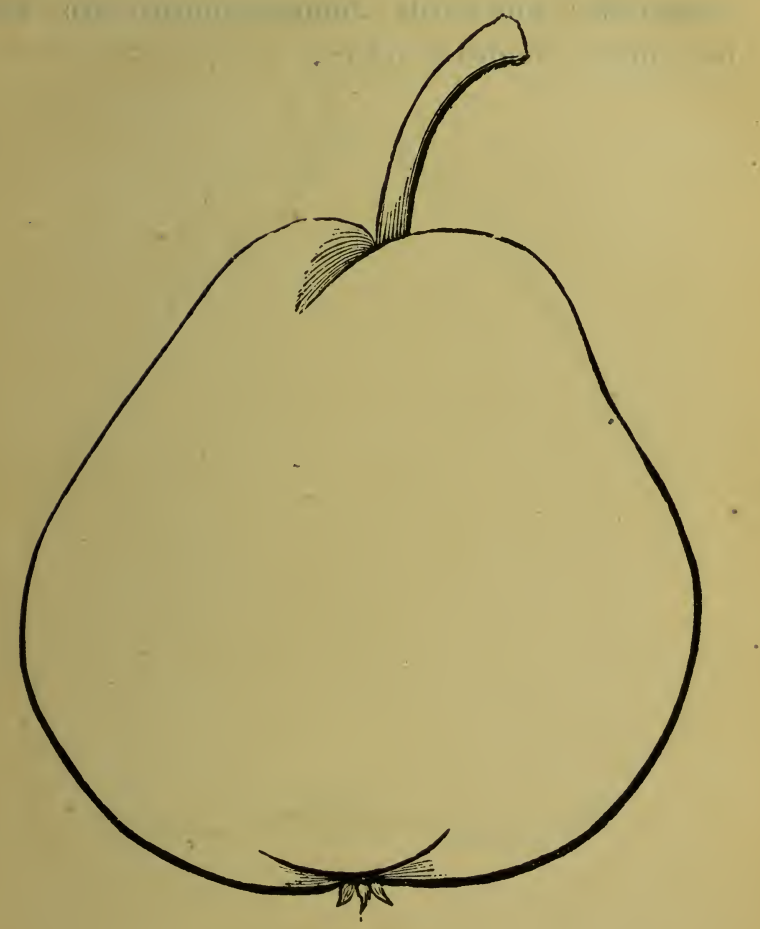

Beurré Millet.

Fruit moyen; peau fine, vert clair, tiquetée de points roux, tantòt tachée de carmin du côté du so- 
leil. Chair très-fine, beurrée, fondante, juteuse et d'un parfum relevé.

Arbre assez vigoureux, mieux sur franc que sur coignassier, très-fertile. Jeunes rameaux gris, feuilles amples, rondes, étoffées. 


\section{No 12}

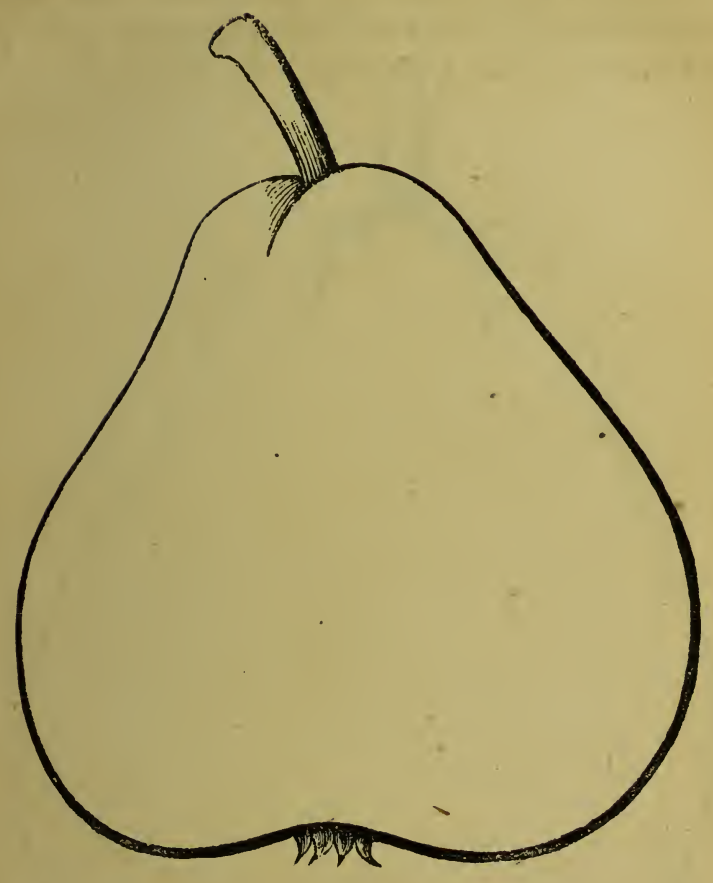

Beurre do Nivelles.

Fruit de moyenne grosseur, en forme de toupie, bossué, peau vert clair, pointillée de rouge, chair ferme, mi-fine, fondante, eau assez abondante et relevée.

Arbre de bonne vigueur, réussit sur franc et sur coignassier. 



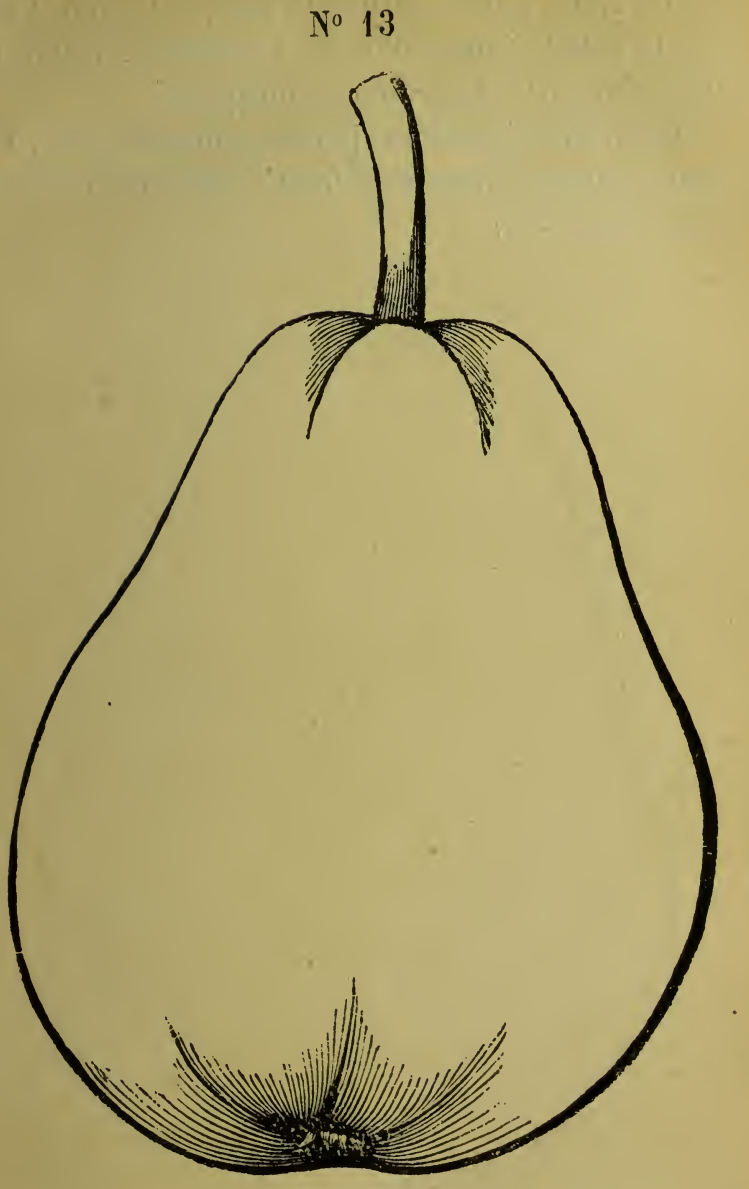

Bon ehrétien Williams (Bartlet de Boston, Barnet's, Bon chrétien Barnet's, Williams Pear).

Fruit gros, bossué, ventru, peau vert clair, tique- 
tée de roux, parfois striée de rouge du côté éclairci par le soleil, jaune d'or à la maturité. Chair trèsfine, fondante, légèrement musquée.

- Arbre très-vigoureux, jeunes rameaux, gros, forts, verts, feuilles larges vert foncé. Réussit sur franc et sur coignassier. 
$\mathrm{N}^{\circ} 14$

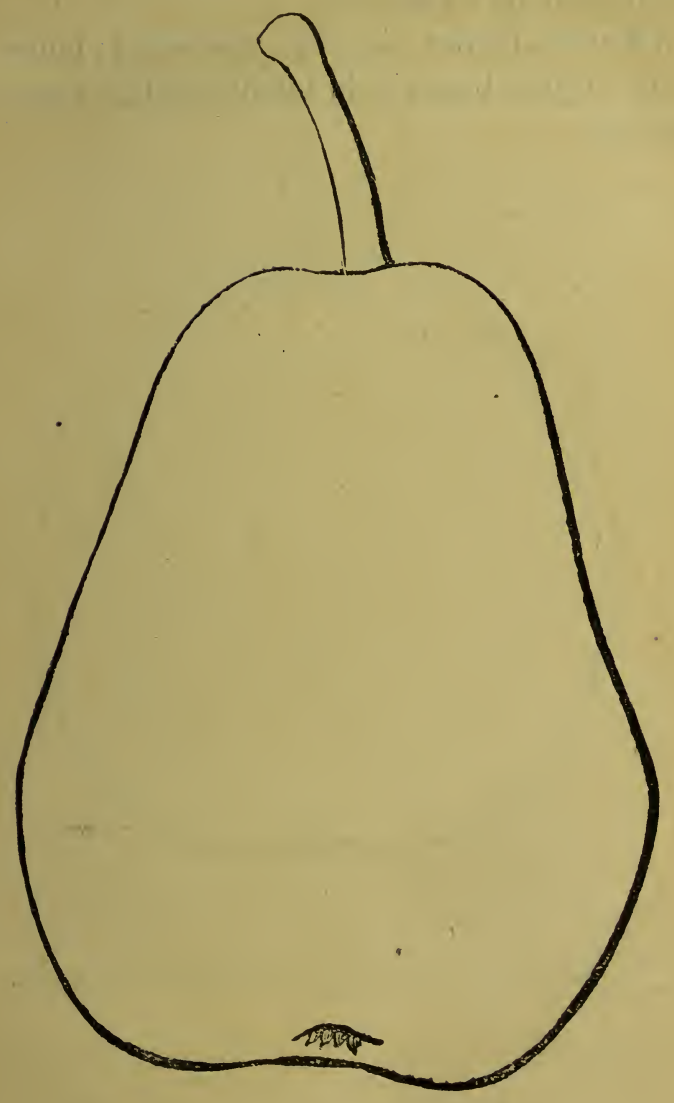

Bonne d'Émê.

Fruit gros, long, presque cylindrique, renflé au 
milieu ; peau vert clair, tiquetée de gris roux. Chair fine, fondante, sucrée, juteuse et d'une saveur rap. pelant celle de l'amande.

Arbre de bonne vigueur, très-fertile; jeunes rameaux bruns tiquetés de blanc, feuilles grandes et entières. 


\section{$\mathrm{N}^{\circ} 15$}

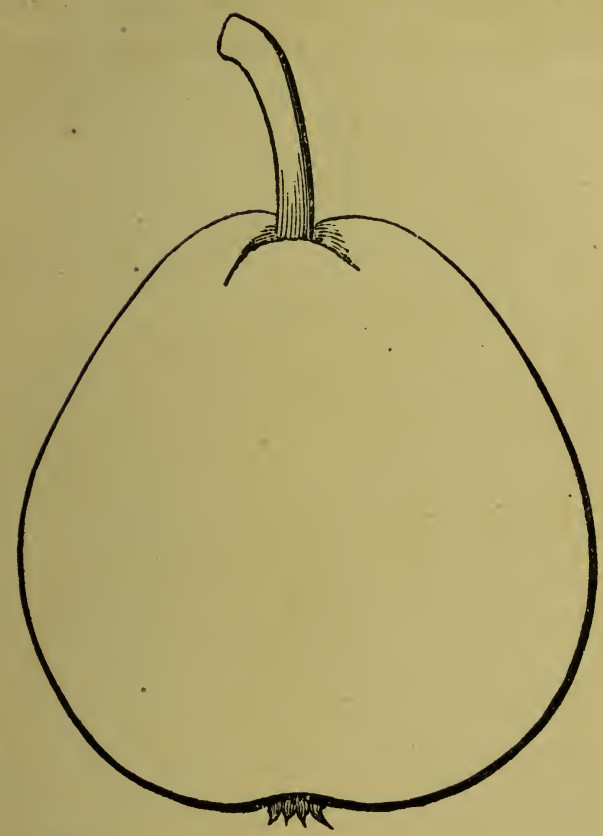

Bonne de Maline (Colmar Nélis, Nélis d'hiver).

Fruit petit, assez régulier; peau vert brun, tiquetée de même couleur, devient d'un jaune rouge à la maturité. Chair blanche, très-fondante, beurrée; eau peu abondante et parfumée. L'une des meilleures poires qui existent. 
Arbre de moyenne vigueur, ne réussissant bien que sur franc dans la plupart des terrains; rameaux minces, petits, feuilles étroites. 
$\mathrm{N}^{\circ} 16$

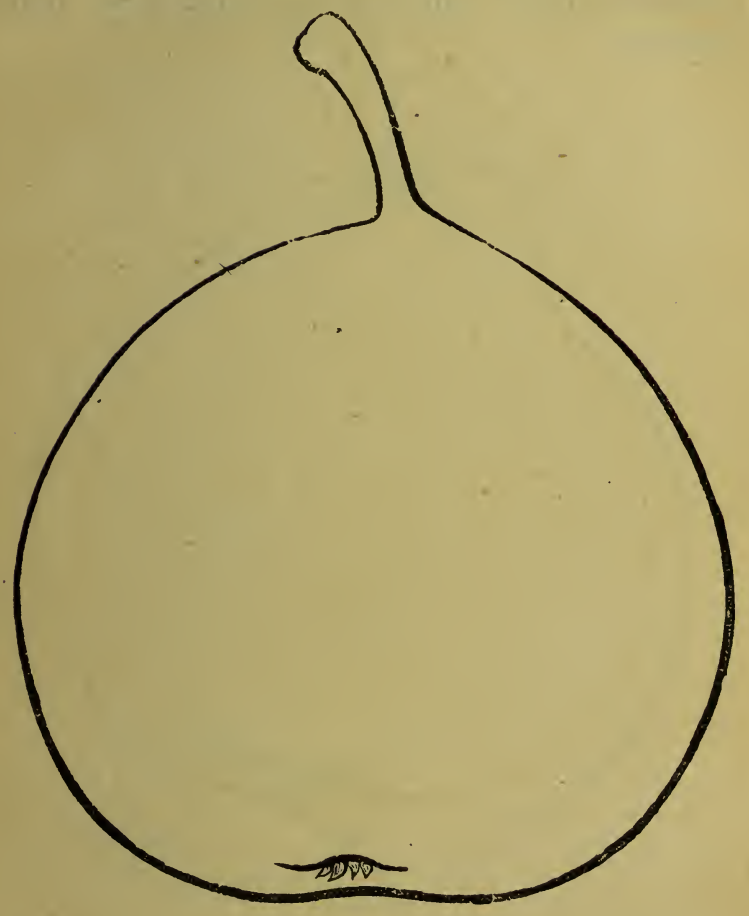

Bergamotte Espéren.

Fruit moyen; peau rude, verte, tachée de roux, rarement fouettée de rouge du côté du soleil, jaunissant un peu à la maturité. Chair blane rosé, fine, 
fondante, juteuse, sucrée et bien parfumée. C'est le meilleur des fruits de longue garde.

Arbre d'une grande vigueur; très-fertile, un peu épineux. Jeunes rameaux vert rougeâtre; feuilles grandes. 


\section{$N^{\circ} 17$}

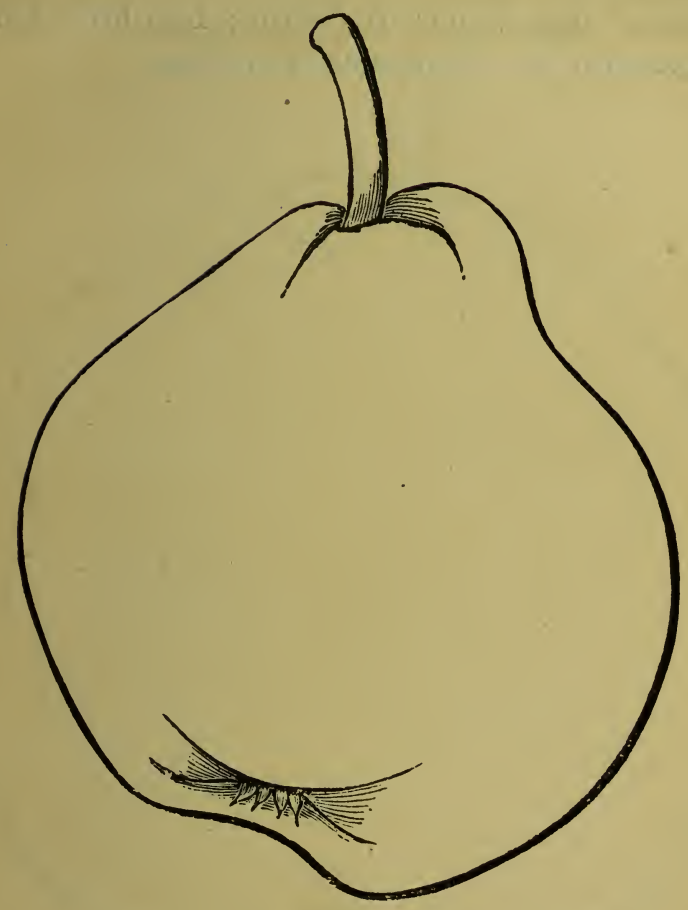

Bergamotte fortunée (Fortunée).

Fruit de moyenne grosseur, irrégulier, trapu; peau très-rude, vert foncé, tachée de rouille, tiquetée de points bruns et gris; chair fine, mi-fondante, assez juteuse, relevée; parfois pierreuse au centre. 
Ce fruit est précieux parce qu'il se garde jusqu'en mai, et qu'il est également bon pour la table et pour cuire.

Arbre assez vigoureux, fertile; bois brun foncé, réussissant sur coignassier et sur franc. 
teinté de rouge. Chair cassante, granuleuse, eau presque nulle; se conserve jusqu'en mai.

Arbre vigoureux, très-fertile, aussi bien sur franc que sur coignassier. 


\section{No 19}

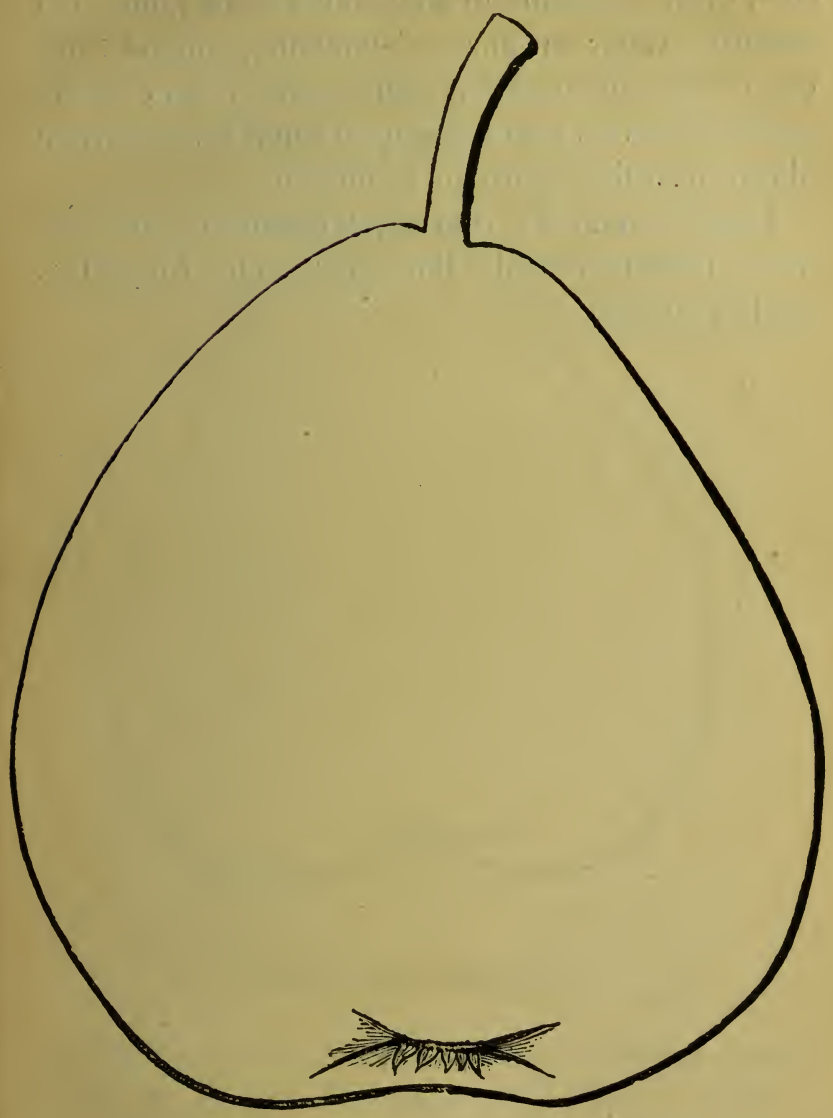

Colmar d'A remberg.

Fruit gros ou très-gros, renflé dans le milieu, 
côtelé parfois ; peau fine, vert jaunâtre, lisse, tiquetée de brun, passant au roux fauve et au jaune à la maturité. Chair mi-fine, mi-fondante, juteuse, relevée. C'est un excellent fruit, mais il perd de ses qualités dans les terres froides et humides et devient plutôt un fruit à cuire qu'à couteau.

Arbre de peu de vigueur, exigeant la greffe sur franc; il est très-fertile. Bois gros, court, rougeâtre; feuilles étoffées. 


\section{PREMIÈRE PARTIE}

\section{$\mathrm{N}^{\circ} 20$}

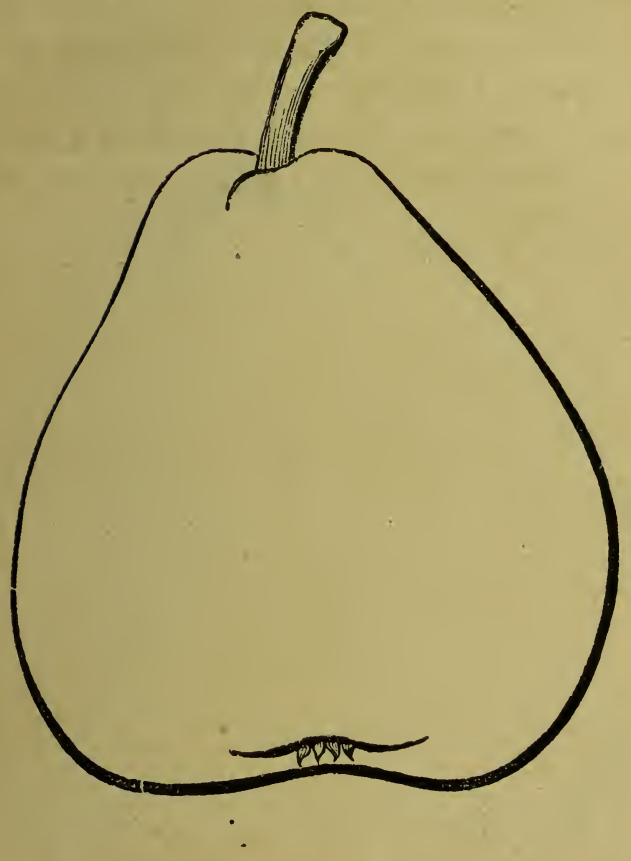

Commissaire Delmotte.

Fruit de grasseur moyenne; vert sombre; peau chagrinée, tiquetée de gris; chair ferme, mi-fine, mi-fondante, eau abondante et parfumée.

Arbre vigoureux, réussissant sur franc et sur coignassier; d'une bonne fertilité, quoiqu'un peu lent à se mettre à fruit. 



\section{No 21}

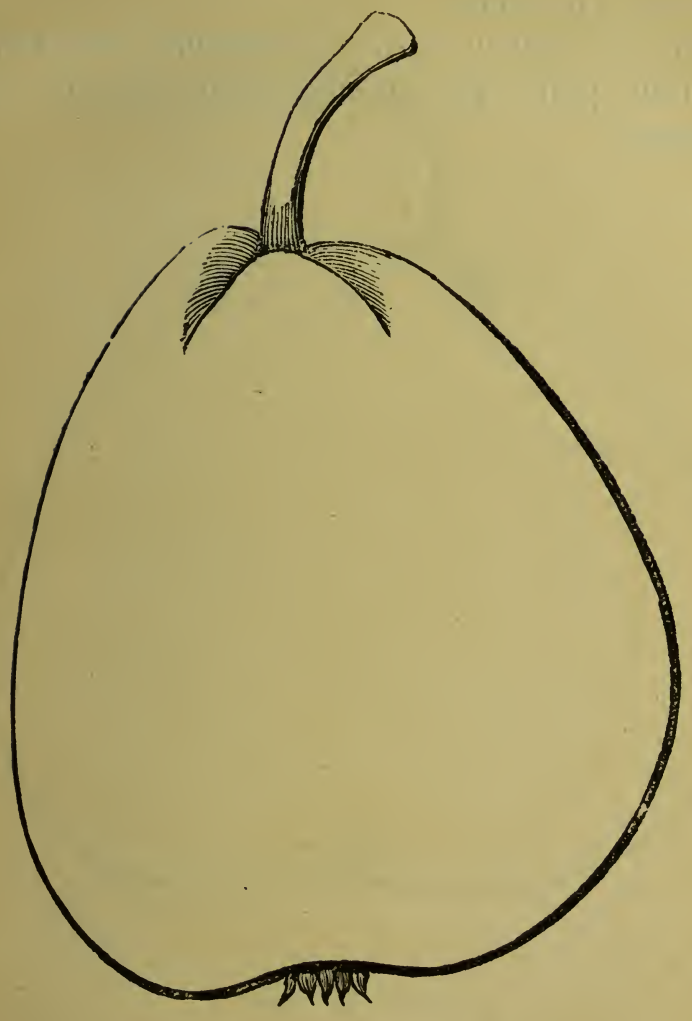

Délices de Louvenjoul (Syn. : Jules Bivorl).

Fruit de moyenne grosseur, ovale tronqué; peau vert clair; lisse, tiquetée de gris et de rouille, se colo- 
rant du côté frappé par le soleil, et devenant jaune à la maturité ; chair fine, fondante, juteuse, sucrée, vineuse et parfumée.

Arbre de vigueur moyenne, demande à être greffé sur franc plutôt que sur coignassier; rameaux vert rougeâtre. 
No 22

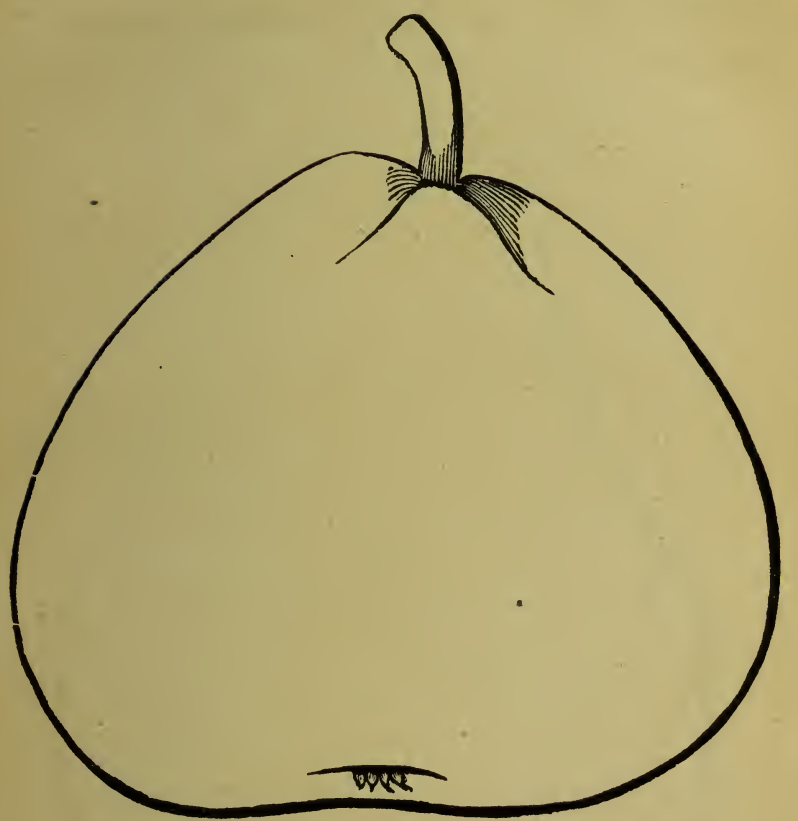

Polre Devergnies.

Fruit gros, rappelant la forme des bergamottes; peau vert clair, fortement tachée de rouille, jaspée vermillon du côté frappé par le soleil, passant au jaune d'or à la maturité. Chair fine, fondante, jau- 
nâtre; eau abondante, sucrée et parfumée. Ce fruit se conserve jusqu'en février.

Arbre assez vigoureux sur franc et sur coignassier ; il est fertile. 
No 23

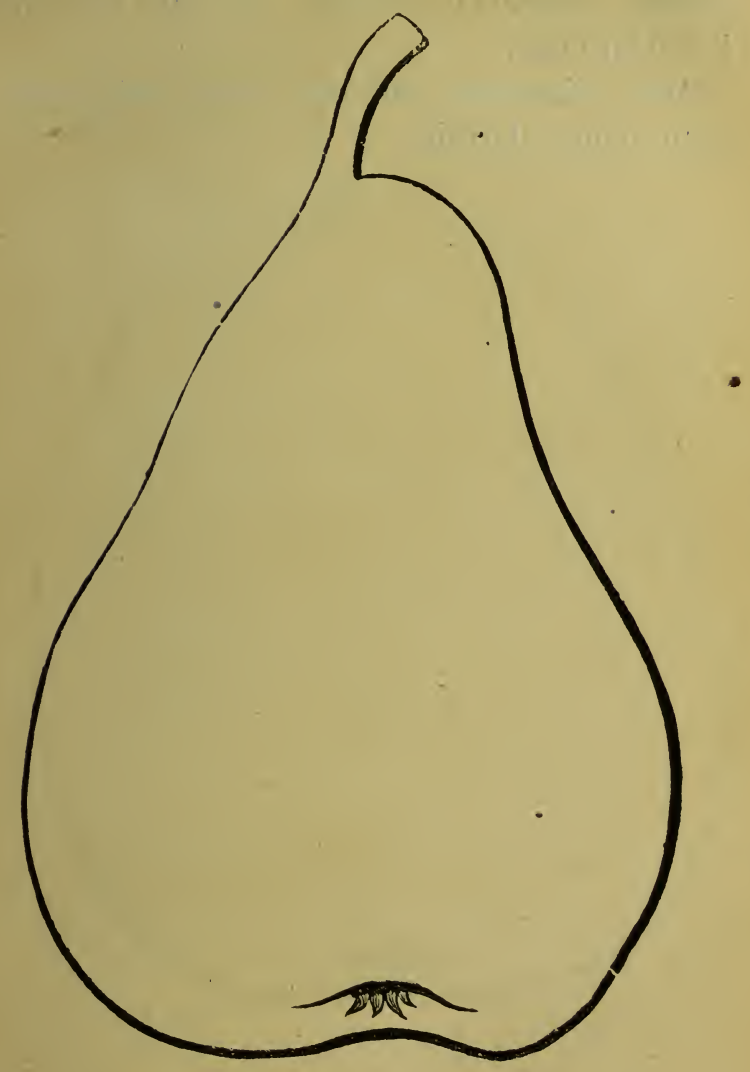

Docteur Bonvier.

Fruit gros, allongé, pyriforme ; peau vert clair.

6. 
maculée de roux et de brun, passant au jaune à la maturité. Chair demi-beurrée, mi-fondante, trèsjuteuse, sucrée et parfumée. Ce fruit se conserve jusqu'en mars.

Arbre vigoureux sur franc et sur coignassier et d'une bonne fertilité. 
PREMIĖRE PARTIE

103

No 24

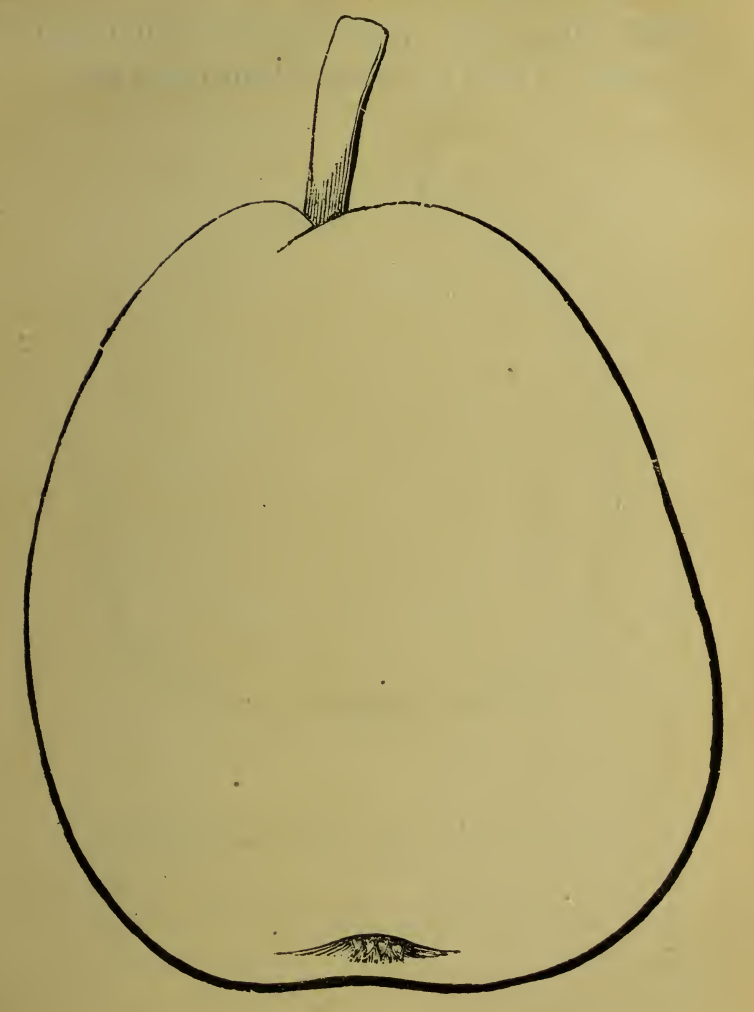

Doyennd d'Alençon (Doyenné d'hiver nouveau).

Fruit assez gros, généralement allongé, parfois arrondi, renflé au milieu; peau verdatre, tiquetée 
de roux; chair fine, fondante, tirant sur le jaune: eau abondante, sucrée, relevée ou acidulée. Ce fruit se conserve jusqu'en février.

Arbre vigoureux, s'accommodant du coignassier et du franc, fertile; rameaux brun rougeattre. 


\section{No2:)}

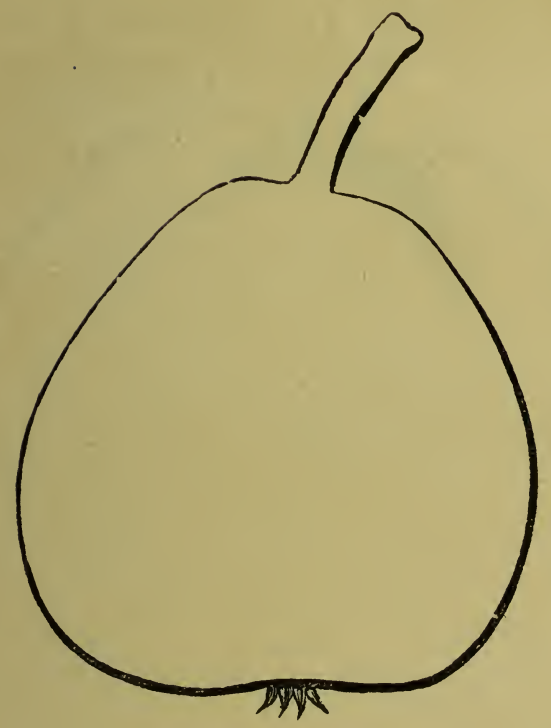

Doyenné de julllet (Syn. : Roi Jolimont).

Fruit petit, ovale ou légèrement turbiné-obtus; peau lisse, vert citron, colorée au soleil, devient rouge de ce côté et jaune vif de celui de l'ombre. Chair demi-fine, neigeuse, presque fondante; eau abondante, sucrée et parfumée. Son plus grand mérite est dans sa précocité.

Arbre de moyenne vigueur, très-fertile, mieux sur franc que sur coignassier. 

No 26

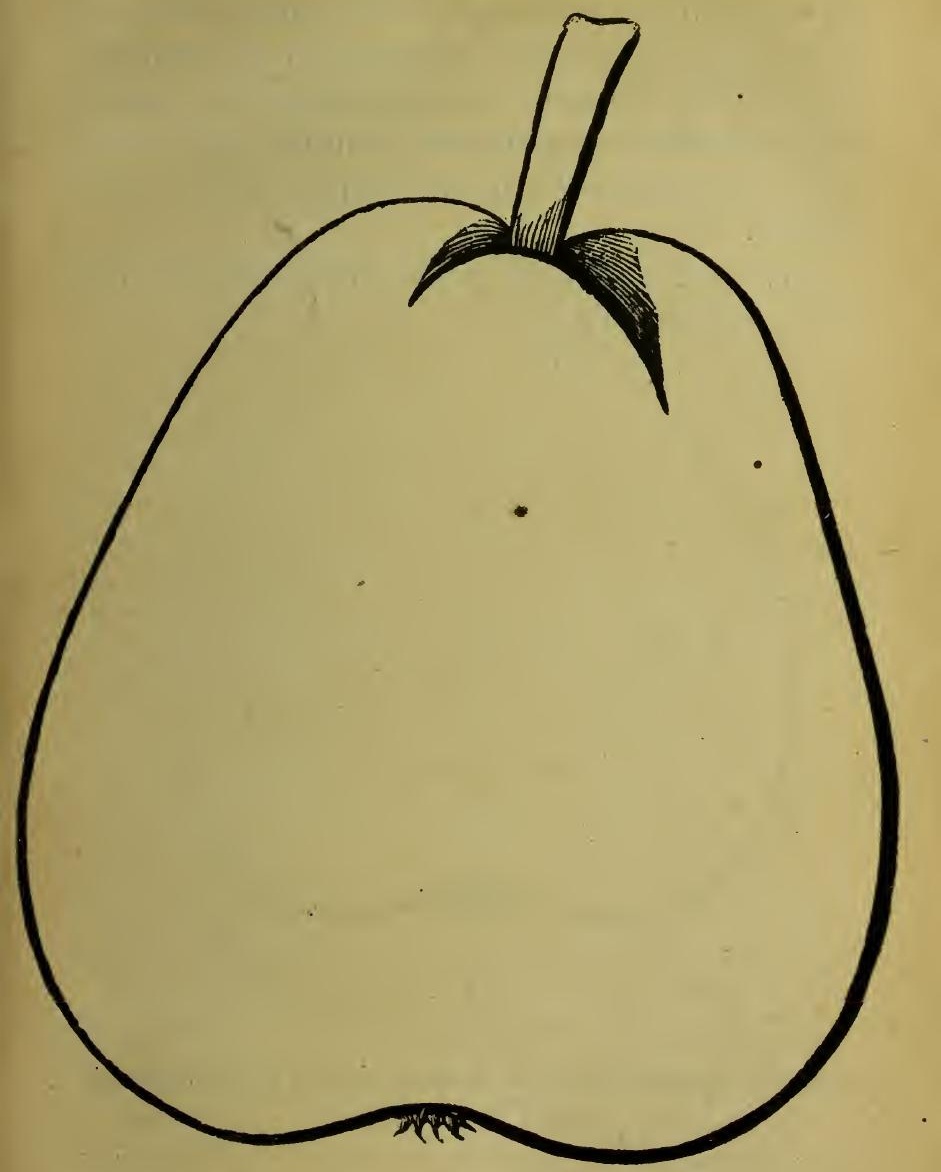

Duchesse d'Angoulome (Syn. : Duchesse).

Fruit très-gros, bossué, ventru, obtus aux extré- 
mités; peau épaisse, vert clair ou jaune citron, rugueuse, tiquetée de gris fauve; parfois lavée de rouge du côté du soleil, jaunissant à la maturité. Chair mi-fine, fondante; eau très-abondante, généralement sucrée et parfumée. L'un des plus beaux fruits, se conservant souvent jusqu'à la fin de décambre.

Arbre très-vigoureux et très-fertile, rameaux vert clair; feuilles larges. 


$$
\text { No } 27
$$

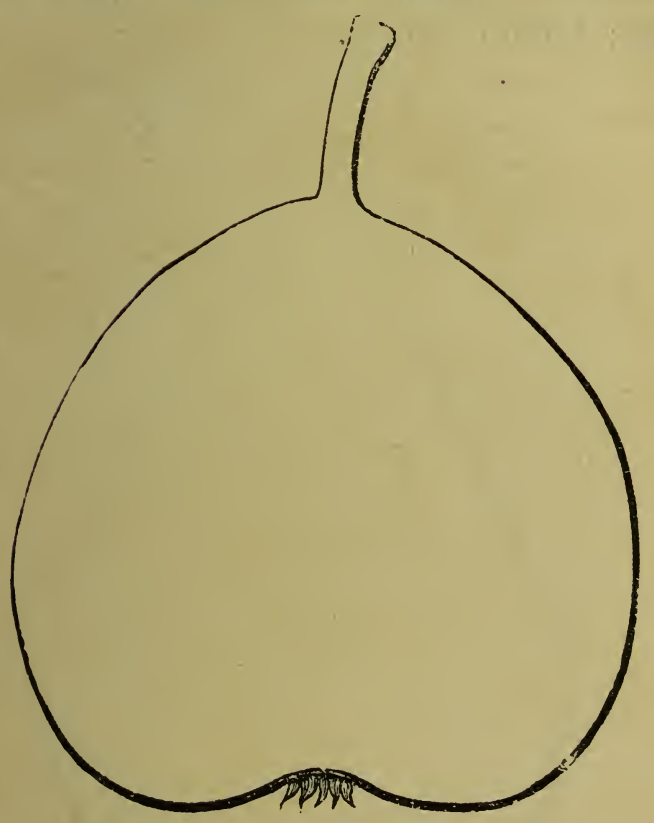

Duchesse de Berry d'été (semble être synonymo de Duchesse de Berry, de Nantes).

Fruit petit ou moyen, imitant la forme des doyennés; peau lisse, vert très-clair, tiquetée de 
blanc, parfois rouge vermillon du côté du soleil. Chair cassante, juteuse, sucrée, parfumée. Arbre très-vigoureux, sur franc et sur coignassier, très-fertile; rameaux forts, verts, tirant sur le rouge; feuilles grandes. 
No 28

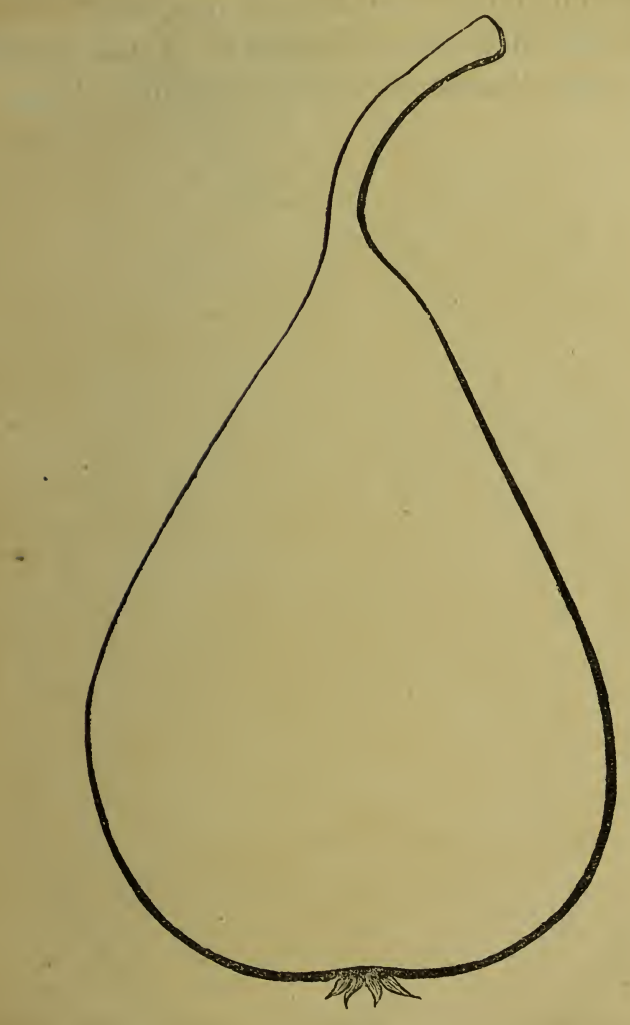

Epargne (Syn. : Beau prẻsent, Cuisse madame, Saint-Samson Cuillette).

Fruit moyen, allongé, quelquefois irrégulier; peau 
verte, tiquetée de fauve et de rouille, jaunissant à la maturité. Chair mi-fine, un peu pierreuse au centre; eau peu abondante, relevée, peu parfumée.

Arbre très-vigoureux, très-fertile, à rameaux divariqués, gros, lisses, vert foncé; feuilles larges et étoffées. 


\section{No 29}

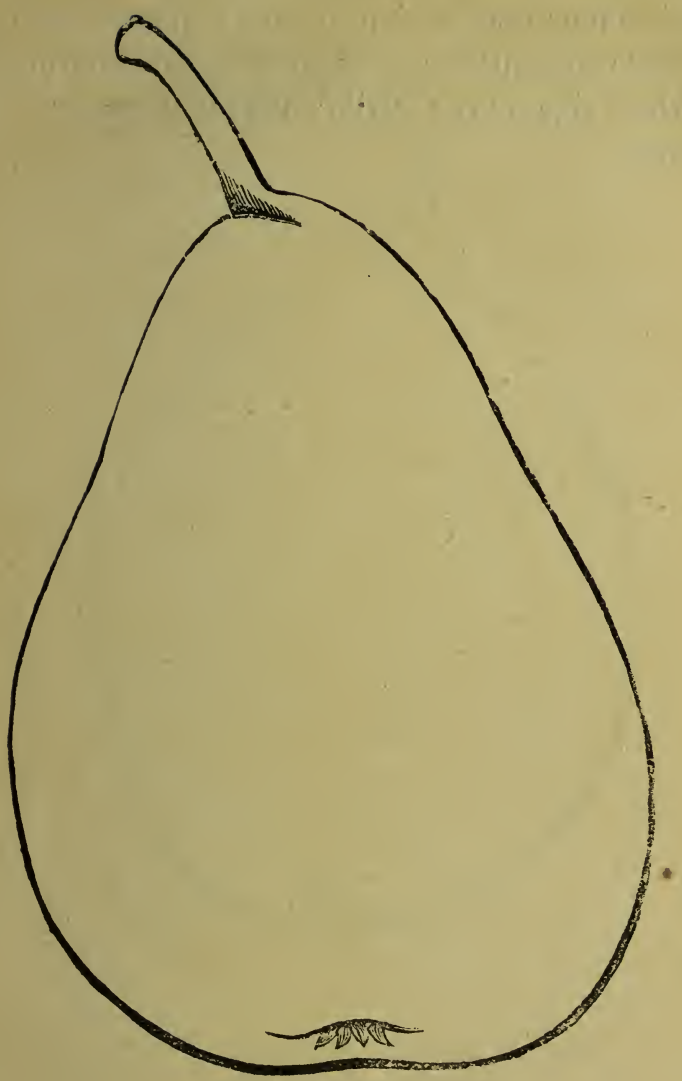

Épine da Mas (Colmar du Lot, duc de Bordeaux).

Fruit dé moyenne grosseur, pyriforme; peau 
lisse, luisante, vert tendre, tiquetée de roux et brun, colorée du côté du soleil, passant au jaune à la maturité. Chair blanche, tirant sur le jaune, mi-fine, fondante, très-juteuse, très-sucrée et très-parfumée. Arbre vigoureux, fertile; jeunes rameaux rougeâtres. 


\section{No 30}

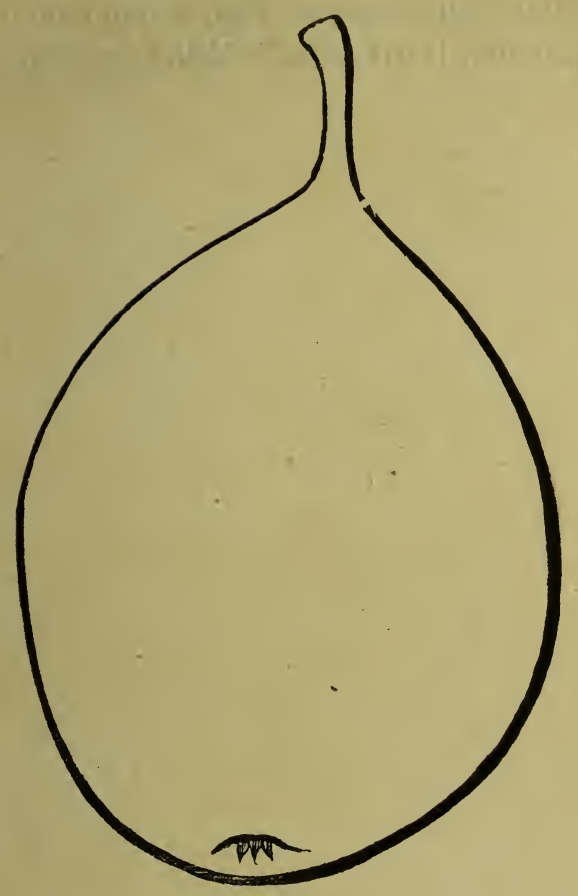

Franceline Millot.

Fruit moyen, oviforme; peau rude, de couleur rousse; chair tirant sur le jaune, demi-fine et cassante, passablement juteuse, très-sucrée et parfu- 
mée. Ce fruit a le triple mérite d'être bon cru, excellent cuit et de se conserver jusqu'en mai.

Arbre de bonne vigueur et très-fertile. Ce poirier n'est connu que depuis 1830 , époque de son premier produit; il est da à M. Millot, de Nancy. Trèsrare. 


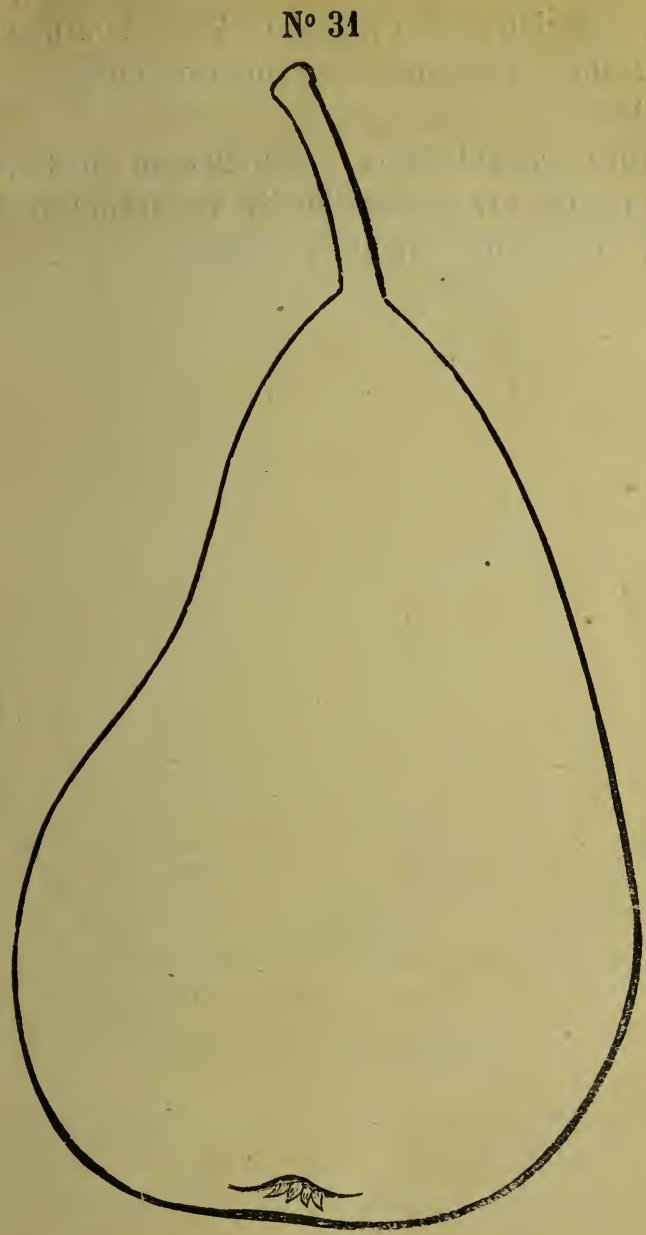

Frédéric de Wurtemberg (Syn. : Médaille d’or).

Fruit de bonne grosseur, pyriforme, long; peau 
fine, vert clair, passant au jaune d'or à la maturité; chair fine, fondante, très-juteuse, sucrée et parfumée.

Arbre passablement vigoureux et très-fertile, réussissant sur coignassier et sur franc; rameaux longs, rougeâtres ; feuilles étroites. 


$$
\text { No } 32
$$

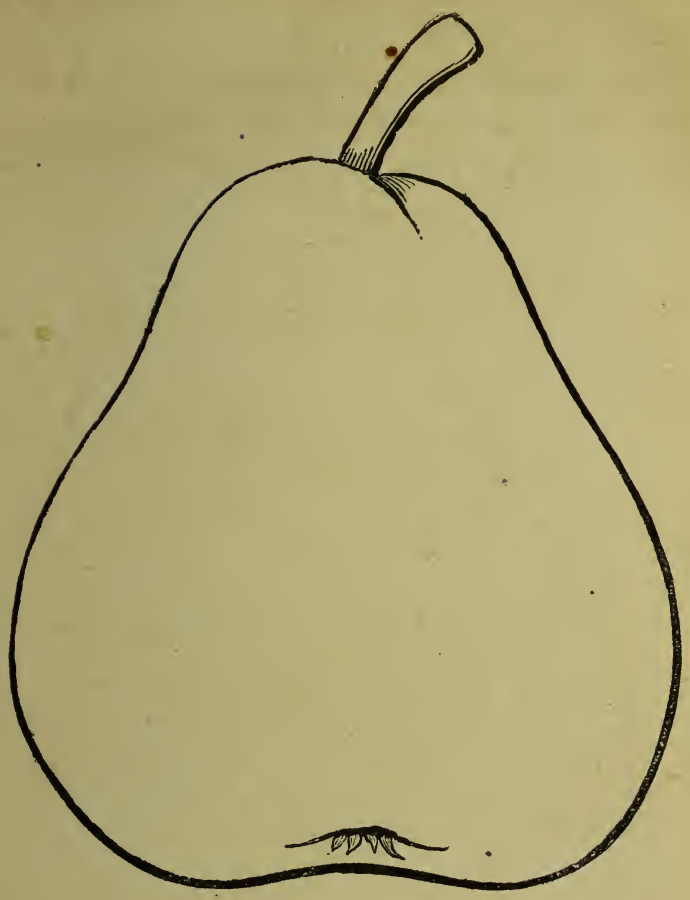

\section{Joséphine de Mallines.}

Fruit moyen, presque rond, aussi large que haut; peau jaune verdâtre, lisse, épaisse, tiquetée de rouille; chair très-fine, fondante; eau abondante, sucrée et parfumée.

Arbre vigoureux sur franc, faible súr coignassier, fertile ; rameaux rouges, gros ; feuilles étroites. 



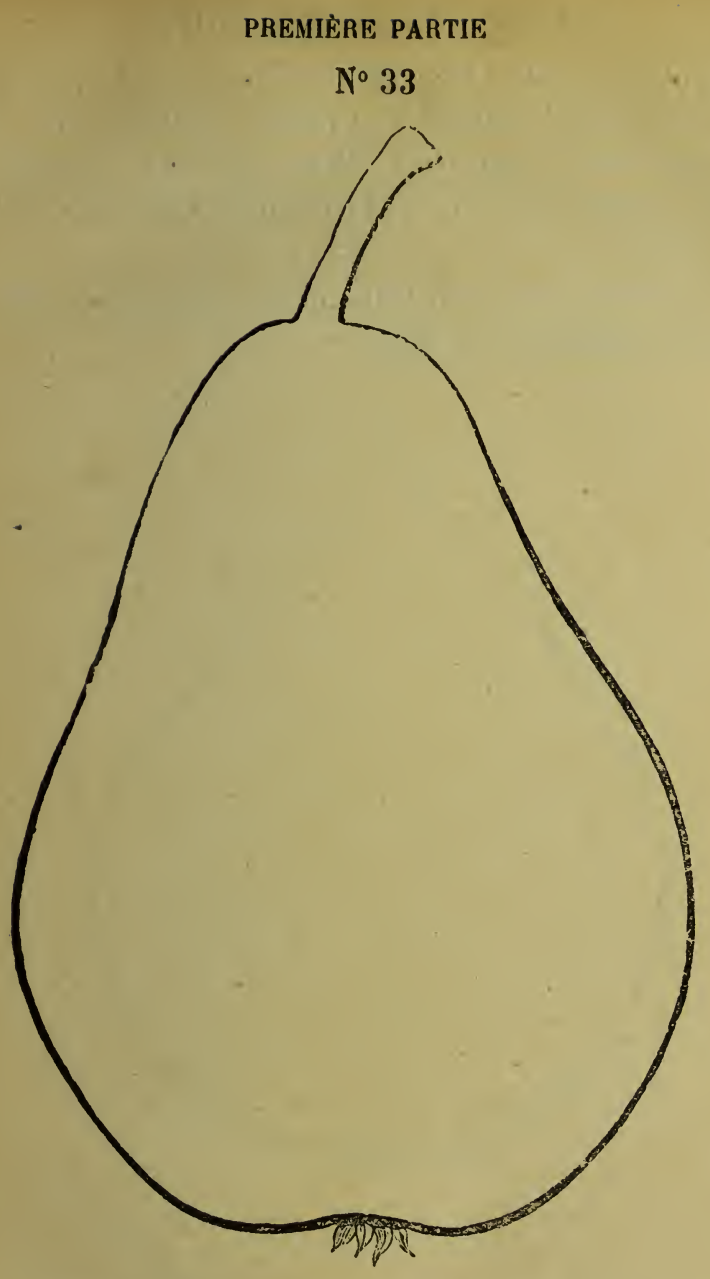

Loulgo Bonne d'Avranches (Louise de Jersey, Bonne de Longueval).

Fruit assez gros, pyriforme, allongé, ventru; peau 


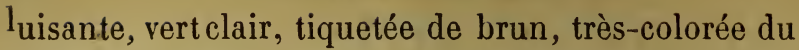
côté du soleil, passant au jaune et au carmin au moment de la maturité; chair très-fine, fondante, pleine d'une eau relevée et parfumée. C'est un excellent fruit.

Arbre vigoureux sur franc et sur coignassier, trèsfertile; rameaux vert foncé. 


\section{No 34}

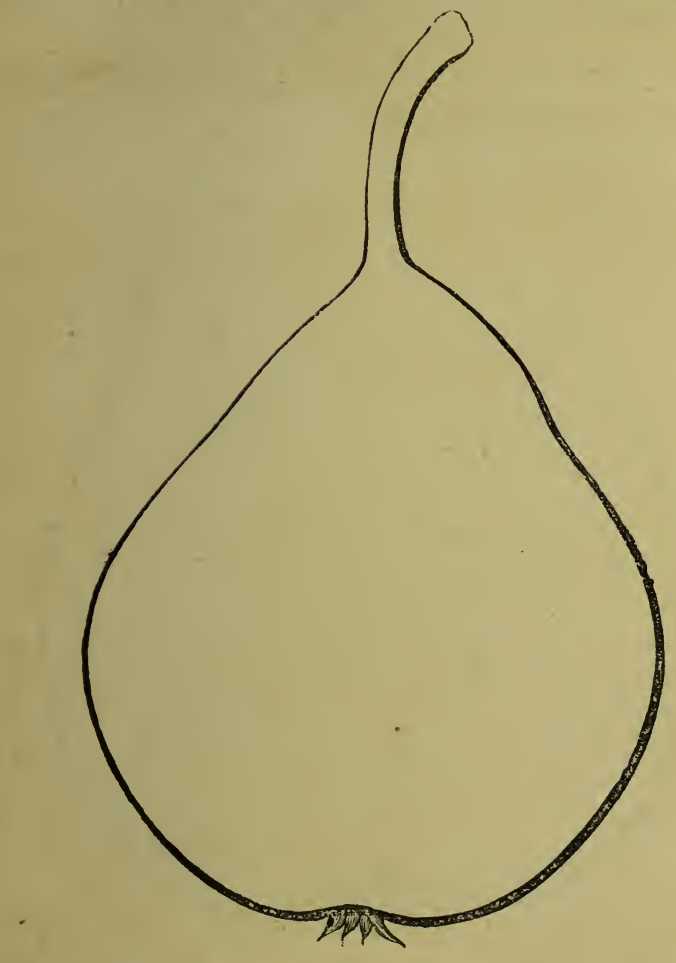

Martin sec (Syn. : Rousselet d'hiver).

Fruit petit ou moyen, en forme de calebasse ; peau vert jaunâtre, tiquetée de blanc et maculée de brun; 
chair assez fine, cassante, sucrée. Ce fruit se conserve bien; mais il n'est bon que cuit. C'est l'un des meilleurs pour compote.

Arbre vigoureux, fertile, surtout propre pour plein vent. 
No 3 מ

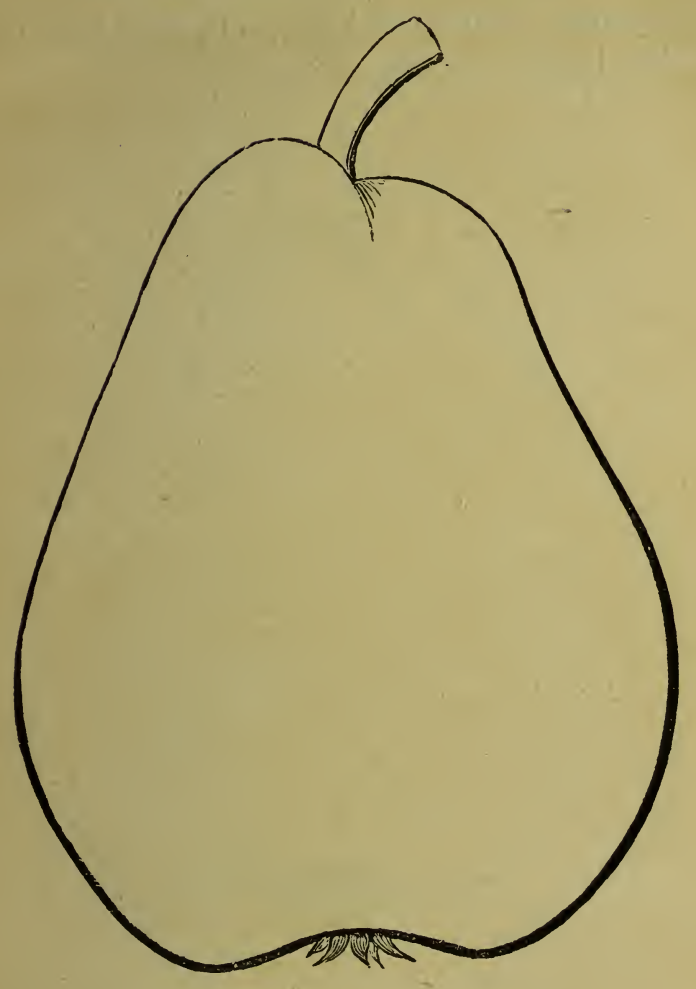

Nee plus Meurls (Syn. : Beurré d'Anjou).

Fruit gros ou assez gros, obovale, parfois irrégulier, généralement renflé dans le milieu; peau vert clair, unie, tiquetée et maculée de fauve, jaunit en 
mûrissant ; chair fine, fondante, juteuse, vineuse, relevée, parfumée.

Arbre assez vigoureux, réussit sur franc et sur coignassier ; fertile par l'âge ; rameaux brun clair ; feuilles larges, vert pâle. 
$\mathrm{N}^{\circ} 36$

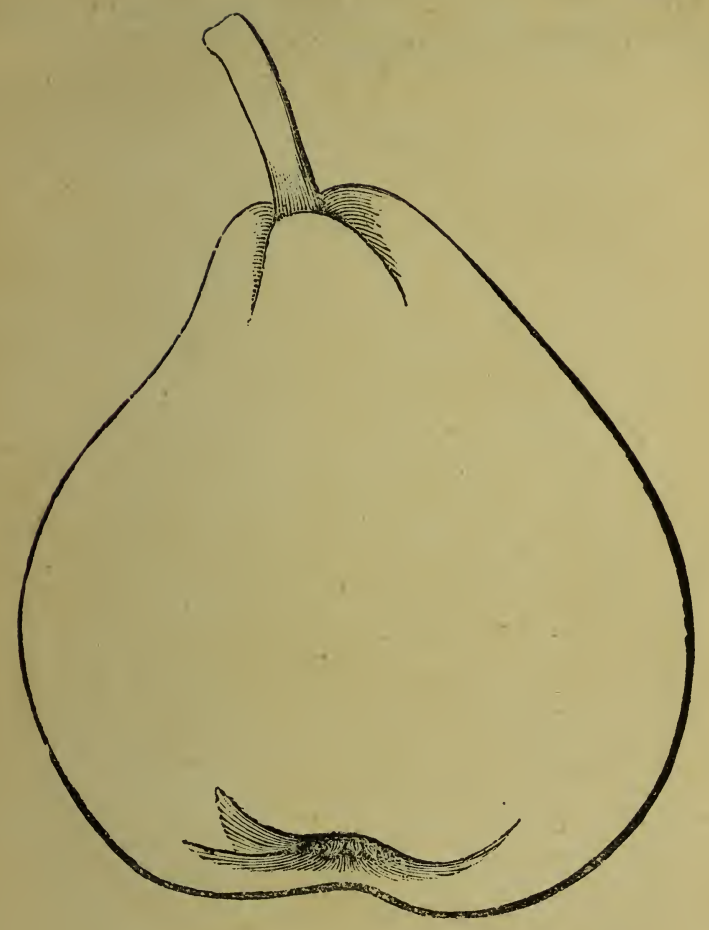

Passe-Colmar (Passe-Colmar gris ou doré).

Fruit gros ou moyen, pyriforme; peau vert clair, tiquetée de brun et de roux; chair ferme, fine, fon- 
dante; eau suffisante, très-sucrée, délicieusement parfumée, l'une des meilleures poires qui existent.

Arbre peu vigoureux, très-fertile, exige le franc; jeunes rameaux rougeâtres. 
No 37

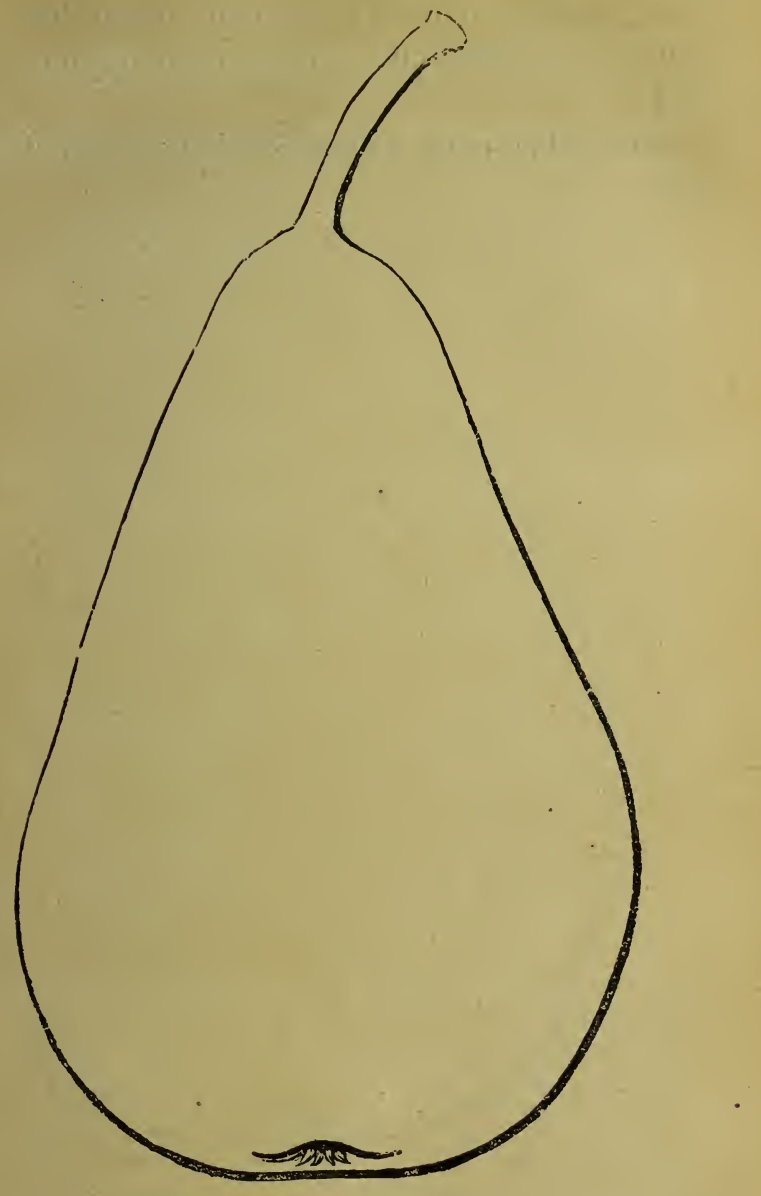

Cros Ronsselet d'aout.

Fruit de moyenne grosseur, pyriforme, allongé; 
peau vert clair, tiquetée de brun, maculée de carmin, devient jaune d'or à la maturité ; chair fine, fondante; eau abondante, sucrée, d'un parfum excellent.

Arbre vigoureux, réussit sur franc et sur coignassier. 


\section{PREMIÈRE PARTIE}

$\mathrm{N}^{\circ} 38$

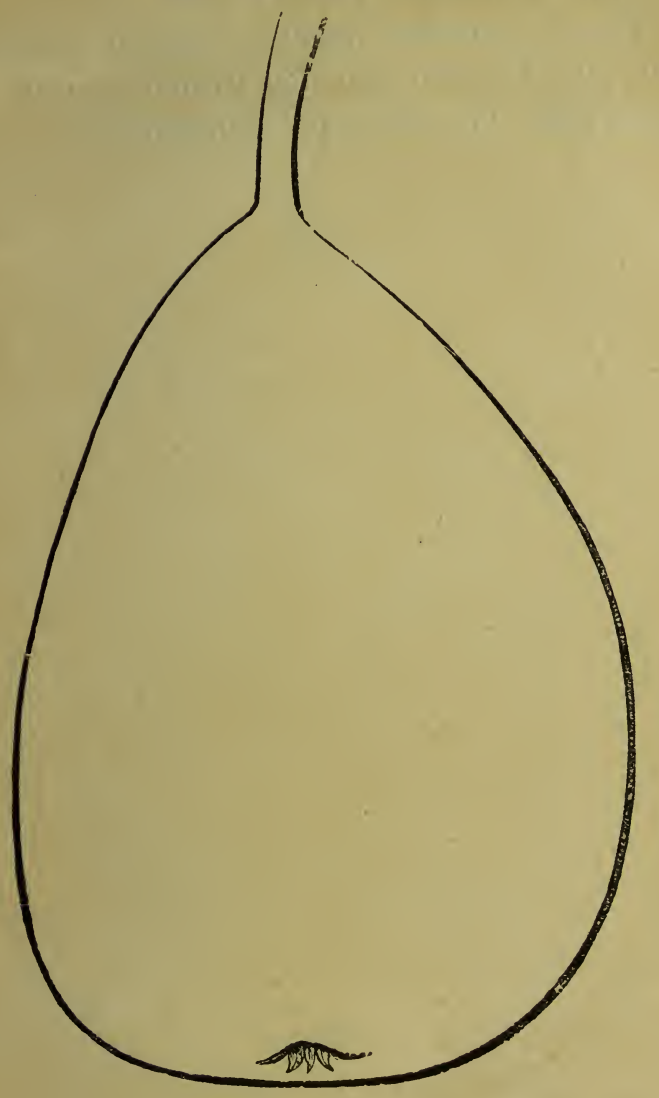

Saint-Herblain d'hiver.

Fruit moyen, pyriforme ; peau unie, vert clair, ti- 
quetée de brun ; chair blanche, fine; eau assez abondante, sucrée. Ce fruit se conserve bien et longtemps, ce qui le rend très-recommandable. Arbre de moyenne vigueur, exigeant la greffe sur franc pour le plein vent. Cet arbre n'est connu que depuis 1827. Très-rare dans les pépinières. 


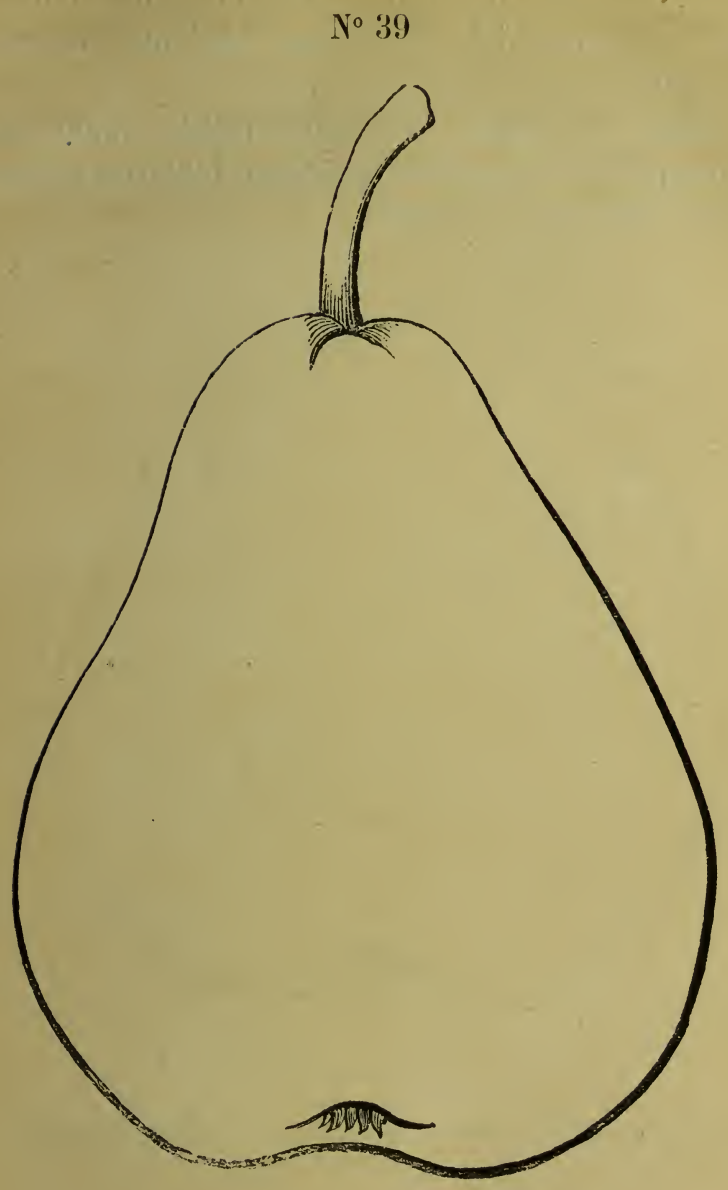

Saint-Michel arehange.

Fruit assez gros ou gros, pyriforme, ventru; peau 8 
unie, vert clair, tiquetée de gris de rouille, jaunissant à la maturité; chair fine, fondante, beurrée, très-juteuse, sucrée et parfumée.

Arbre de vigueur moyenne, fertile, mieux sur franc que sur coignassier, préférant les terres légères; jeune bois brun rougeâtre. 


\section{No 40}

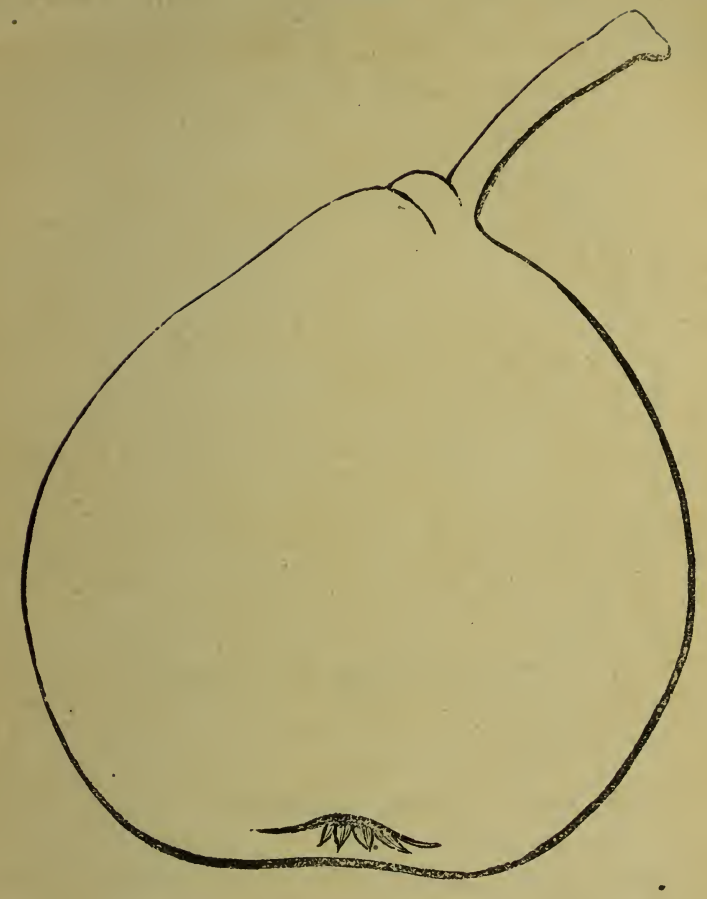

Selgneur (Syn. : Bergamotte lucrative, Bergamotte fiévée, Fondante d'automne).

Fruit de moyenne grosseur, ayant la forme des bergamottes; peau verdâtre, maculée de roux fauve, 
passant au jaune à la maturité ; chair fine, fondante ; eau abondante, très-sucrée, d'un parfum exquis.

Arbre de moyenne vigueur, très-fertile, ne réussissant bien que sur le coignassier; jeune bois brun rougeâtre. 


\section{No 41}

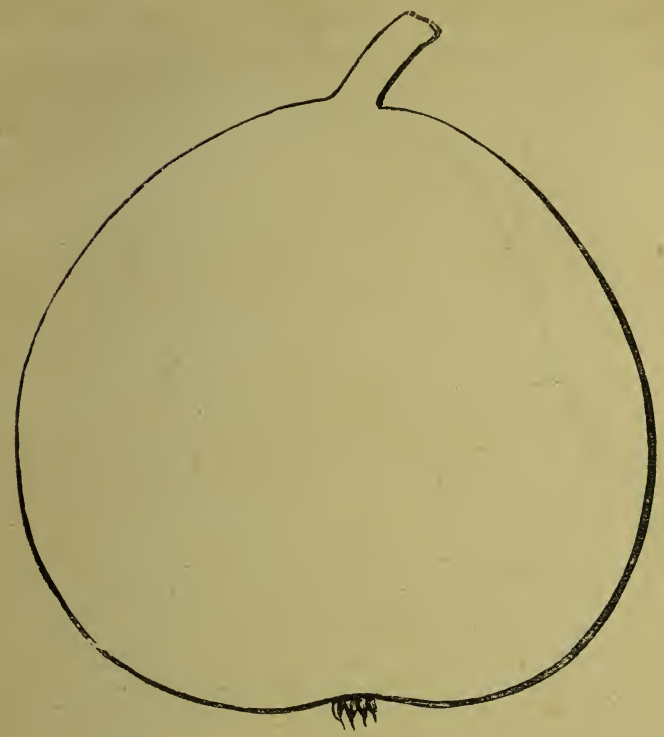

Suzette de Bayay.

Fruit moyen, ovale et bosselé; peau lisse, vert clair, tiquetée de gris, maculée de roux, jaunissant à la maturité; chair tirant sur le vert, fondante ; eau abondante, sucrée et parfumée. Ce fruit est précieux en ce que, indépendamment de ses qualités, il se conserve jusqu'en avril.

Arbre vigoureux, passablement fertile, réussissant sur coignassier et sur franc, même à haute tige. 



$$
\text { No } 42
$$

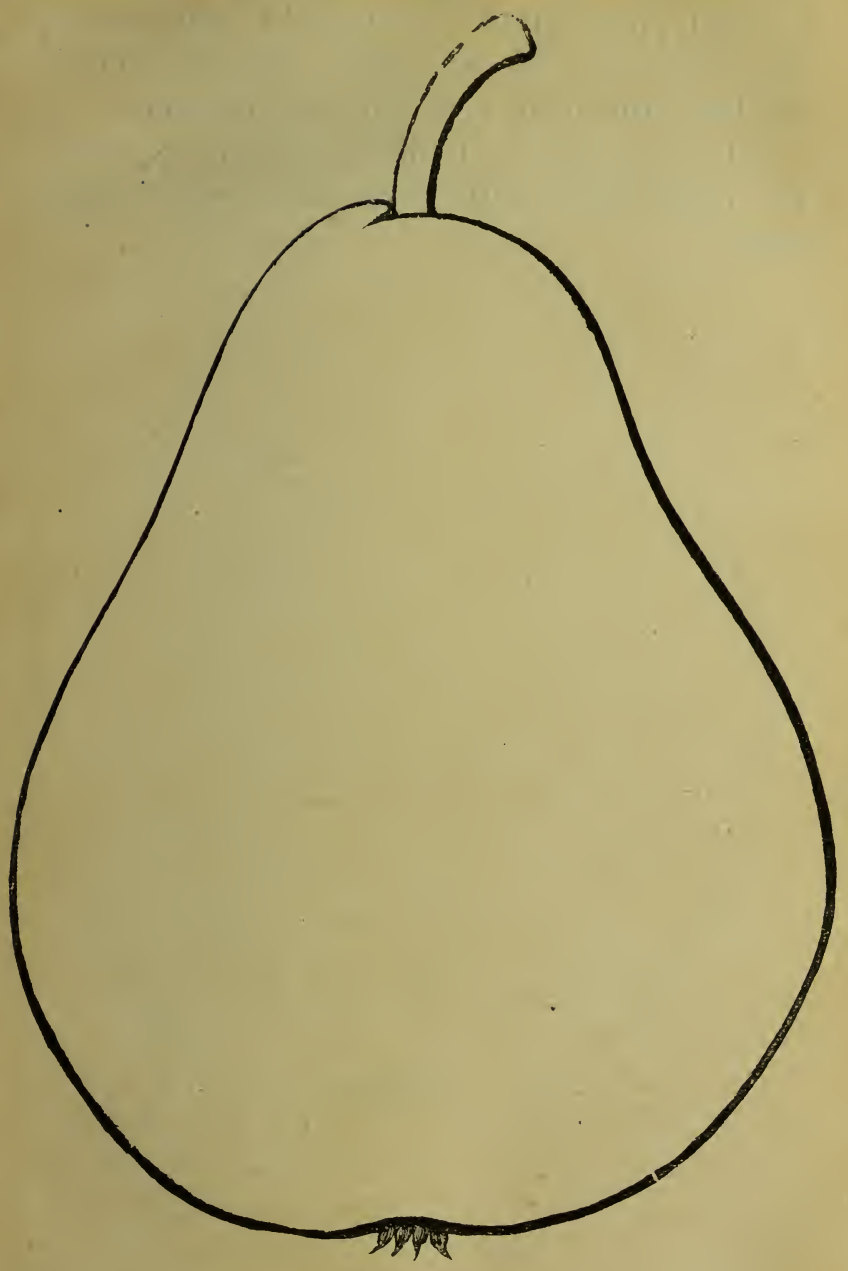

Triomphe de Jodolgne.

Fruit gros ou très-gros, pyramidal, ventru, bos- 
selé ; peau fine, grasse, luisante, vert clair, maculée de brun, jaune citron à la maturité ; chair beurrée, fine, fondante; eau abondante et très-parfumée; excellent fruit, mais se gardant peu de temps.

Arbre vigoureux et fertile, réussissant sur franc et sur coignassier; jeune bois gros, brun; feuilles larges. 


\section{No 43}

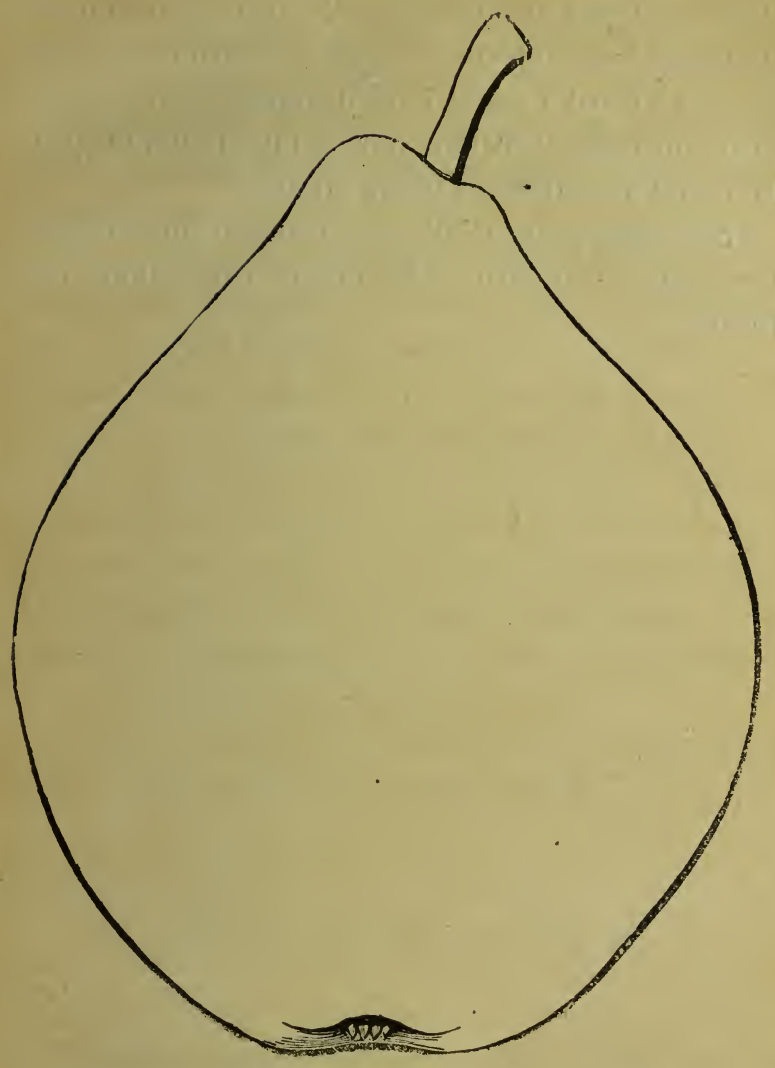

Vauquelia.

Fruit assez gros, allongé, imitant un peu le coing; 
peau épaisse, rude, vert jaunâtre, maculée de taches gris roux, jaunissant à la maturité ; eau abondante, sucrée, relevée et parfumée. Ce fruit se rapproche beaucoup comme forme, couleur et saveur du Saint. Germain; il est à désirer qu'il puisse le remplacer. Il est, du reste, précieux par sa longue garde, puisqu'il peut se conserver jusqu'en mai.

Arbre vigoureux, fertile sur franc et sur coignassier, mais semblant redouter les expositions trèsfroides. 
DU POMMIER

Le pommier, quoique très-inférieur au poirier, mérite cependant une place au jardin, au verger et aux champs.

La pomme n'est nullement comparable à la poire; cependant, elle a ses amateurs : aussi ne passeronsnous pas sous silence la culture du pommier.

$\mathrm{Au}$ jardin il sert à faire des cordons qui sont promptement à fruit ; c'est une première qualité.

$A u$ verger il fait de beaux arbres pour l'amateur.

Aux champs il rend des services là où le poirier ne réussirait pas ou peu, en offrant à la spéculation un bénéfice moins grand assurément quela poire, mais, cependant encore assez élevé pour n'être pas à dédaigner dans plus d'une circonstance.

Nous ne parlons pas du pommier à cidre, cela ne rentre pas dans le cadre de cet ouvrage.

DU SOL CONVENABLE AU POMMIER

Le pommier aime de préférence les terres un peu argileuses et fraîches, et croît très-bien dans les terres franches, argilo-calcaires; il réussit aussi dans les sols légers et secs où le poirier ne ferait que végéter. C'est même dans ces sortes de terrains 
qu'il donne les meilleurs fruits; cependant sa place naturelle est dans les terres substantielles et frâ̂ches.

Le sujet sur lequel il est greffé a une grande influence sur sa nature. Ainsi greffé sur franc, il pré. fère les terres franches argileuses et humides. Sur Doucin, il se plaît dans les sols secs, sablonneux et légers. Sur Paradis, il exige une terre forte, substantielle, franche et légèrement fraîche.

Le planteur devra donc tenir compte de ces différences.

Le pommier est peu délicat sur l'exposition; il réussit même au nord et dans des endroits privés de soleil.

Notez que le doucin et le paradis ne doivent être employés que pour les basses tiges et les cordons; mais les pommiers destinés à faire des hautes tiges doivent toujours être greffés sur franc.

PRÉPARATION DU SOL ET PLANTATION

Tout ce que nous avons dit de la préparation du sol et de la plantation à propos du poirier, est applicable au pommier. Il est donc absolument inutile d'y revenir; car nous ne ferions que nous répéter.

Nous passons immédiatement à la composition de la liste des meilleures variétés de pommes à cultiver. 
LES MEILLEURES VARIÉTÉS DE POMMES

Nous allons en avoir bien vite fini avec les bonnes variétés. La liste générale des pommiers est bien au-dessous de celle des poiriers; bien qu'on ait introduit une certaine quantité de nouveautés depuis une quarantaine d'années, presque sans valeur; aussi les omettons-nous avec intention.

Voici la liste des bonnes pommes, par ordre de maturité, ou à peu près; car il est impossible de la fixer d'une manière absolue. Nous admettons une pomme hâtive pour les amateurs, déclarant d'avance qu'elle est sans autre qualité que sa précocité.

Rouge hâtive. .... maturité en juil. et août Paradis, dit pomme de maréchaux en Bourgogne, Pigeonnet rouge en d'autres localités. .... maturité en sept.-octob. De châtaignier. . . . . automne. Reinette du Canada. . . . commencement, hiver. Reinette d'Angleterre. . . hiver.

Reinette grise. ..... id.

Pomme d'or. ...... id.

Reinette Thouin. ... . hiver jusqu'en mai.

Api rose. ...... hiver jusqu'en mai.

Calville blanc. . . . juin.

Pomme de Lestre. . . . . d'une année à l'autre.

(Cette pomme est supérieure à toutes les reinettes ; variété très-rare dans les pépinières.) 
Voilà le bilan des bonnes pommnes; il est court, et nous le trouvons déjà trop long. Si nous nous en tenjons à notre choix, nous le réduirions de près de moitié. Que le planteur y prenne garde; qu'il se défie des nouveautés, à moins qu'il veuille les cultiver à titre de renseignement ou de curiosité.

DU PRUNIER

Le prunier n'a pas une bien grande valeur comme arbre de produit et ses fruits n'ont qu'un faible mérite pour la table; le prunier est inférieur au pommier sous tous les rapports.

Le prunier est, pour ainsi dire, le plus rustique des arbres fruitiers cultivés dans nos jardins et dans les champs

Il se recommande, d'abord par sa rusticité; ensuite, parce que ses produits peuvent se conserver à l'état sec et qu'ainsi ils offrent un certain avantage au cultivateur qui en fait une spéculation.

Quelques variétés sont bonnes comme dessert, d'autres moins bonnes sont très belles; d'autres enfin font d'excellents pruneaux. Nous allons passer en revue ces différentes variétés et les classer par catégories distinctes, afin qu'on puisse savoir à l'avance à laquelle on doit accorder la préférence.

Le prunier se ntel promptement à fruit et réussit dans tous les sols, plus ou moins et sous toutes les formes. 


\section{Irunes pour dessert}

Précoce de Tours ou madeleine maturité. ...... fin juillet.

Monsieur jaune..... mi-août.

Mirabelle petite..... id.

Damas violet ....... août.

Reine Claude de Bavay. . . . août.

Reine Claude. . . . . . a aût.

Reine Claude Diaphane. . . . fin-août.

Tardive musquée. . . . f fin août et courant

septembre.

Coè's Golden dropjaune (la plus

belle de toutes les prunes). septembre.

Mirabelle tardive. .... septembre.

\section{Prunes pour confitures.}

Coès Golden drop jaune, très-belle.

Mirabelle grosse.

- petite.

- tardive.

Reine-Claude de Bavay.

\section{Prunes pour pruneaux}

Diaprée violette, maturité . a aôt. Prune d'Ag^n ...... août septembre.

Dame-Aubert jaune . . . septembre. 
Questhche d'Allemagne ... septembre.

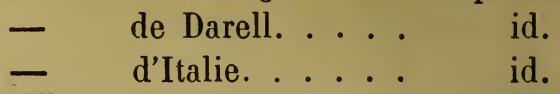

Sainte-Catherine. . . . . .

On distingue encore les prunes pour compotes, mais cette distinction est à peu près insignifiante; car toutes les prunes en général peuvent servir à faire des compotes, notamment celles pour confitures.

CERISIER

Après le poirier, le pommier, le prunier, vient par ordre d'importance le cerisier.

Le cerisier est surtout un arbre de plein vent; cependant il y a quelques variétés qui ne réussissent bien qu'en espalier.

Il réussit dans tous les sols, selon le sujet sur lequel il a été greffé. Sur Sainte-Lucie il s'accommode des terres maigres, peu profondes, sablonneuses, siliceuses et crayeuses. Sur merisier, il lui faut un sol plus profond, plus substantiel, plus consistant, plus frais. Sur sauvageon, il vient bien dans les terres humides, compactes, profondes.

Il prend dans ces dernières conditions un grand développement; aussi doit-on l'espacer beaucoup quand on le plante à plein vent. 
Les meilleures variétés sont :

La Belle d'Orléans hative.

Anglaise hâtive.

Royale.

Impératrice Eugénie.

Montmorency à courte queue.

Belle de Sceaux.

Bigarreau Napoléon.

Anglaise tardive.

RÉCOLTE DES FRUITS

A quel moment de la maturité doit-on cueillir les fruits? Est-ce avant ou au moment de la maturité, est-ce quelques jours après? Telles sont les questions qu'on se pose tous les jours quand on est en présence d'une variété qu'on ne connaît pas très-bien et de longue date.

Certaines variétés de poires demandent à être cueillies plus tôt, d'autres plus tard. Ce n'est qu'à force d'habitude qu'on apprend à connaître le moment opportun pour faire la cueillette : croire qu'on peut le fixer d'une manière invariable est une erreur; l'époque de la maturité varie selon la température, le sol et l'exposition.

On peut poser en principe :

$1^{\circ}$ Que les fruits peuvent et doivent être cueillis environ 15 jours après qu'ils ont cessé de grossir; 20 Que les fruits d'été doivent être cueillis avant 
leur maturité; car ceux qui mûrissent entièrement sur l'arbre, manquent d'eau et de saveur;

$3^{\circ} \mathrm{Qu}$ il est bon et même nécessaire de les cueillir - à plusieurs reprises (c'est ce qu'on appelle entrecueillir), en commençant par les plus avancés d'abord, puis par ceux qui viennent ensuite, pour terminer, enfin par les autres;

$4^{\circ}$ Que les fruits changent de couleur et s'éclaircissent au moment de la maturité, ce qui est un indice certain que le moment de les détacher de l'arbre est arrivé ;

$5^{\circ}$ Que la chute des fruits véreux qui présentent les caractères de la maturité annoncent que le moment de la récolte approche;

$6^{\circ}$ Que pour les fruits d'automne qui ne peuveut se ranger dans le classement ci-dessus, il faut les récolter peu de temps après les fruits d'été. Pourvu qu'ils ne se fanent pas au fruitier, c'est tout ce qu'il faut; car s'ils sont moins parfumés d'abord, ils se conserveront mieux que si on leur eût laissé achever leur maturité sur l'arbre ;

$7^{\circ}$ Que les fruits d'hiver doivent être cueillis le plus tard possible, c'est-à-dire seulement quand les gelées sont à craindre, soit au commencement de novembre, ou à la fin octobre : telles sont les poires qui se conservent jusqu'en février, mars, avril et mai ;

$8^{\circ}$ Que l'on ne doit jamais cueillir un fruit s'il est susceptible de se flétrir ;

9० Que la pratique est le plus sûr guide pur faire la récolte des fruits, et qu'en conséquence on doit 
étudier chaque variété séparément pour plus de certitude, en se conformant aux prescriptions cidessus.

\section{CONSERVATION DES FRUITS}

Nous ne croyons pouvoir mieux faire que de copier textuellement ce que nous avons dit dans l'Horticulteur-gastronome, à propos de la conservation des fruits; car nous n'avons rien à y changer :

a On a indiqué bien des moyens de conserver les fruits; nous pensons qu'il n'y a encore rien de mieux à faire. jusqu à ce jour, que de suivre le procédé décrit par Mathieu de Dombasle. Ainsi donc, plutôt que de faire du vieux neuf, comme cela se voit tous les jours, nous nous bornerons à indiquer ce mode qui consiste à mettre les fruits dans des caisses de 10 centimètres environ de hauteur, de manière à y placer seulement un rang de pommes, poires ou raisins.

»Ces caisses, qui n'ont pas de couvercles, s'empilent les unes sur les autres et se ferment presque hermétiquement réciproquement. Aux côtés de chaque caisse, il y a deux tasseaux qui en dépassent la hauteur de 4 centimètres, afin de servir de guide pour les empiler bien d'aplomb, et aussi pour faire des sortes de poignées pour les manier. Ces caisses faites en bois blane doivent avoir environ 80 centimètres de long, כ̌̋ de large et 10 centimètres de profondeur. 
" Avec une dépense insignifiante, on pourrait faire mieux. Ce serait d'établir une sorte de commode grossière, toujours en bois blanc, avec une série de tiroirs de la dimension ci-dessus. Il n'y aurait que le châssis en sus, et les tiroirs se manieraient plus facilement; car le système de Mathieu de Dombasle a l'inconvénient d'obliger d'enlever les caisses supérieures pour prendre ou visiter les fruits qui se trouvent dans les caisses inférieures. Au moyen de tiroirs cet inconvénient disparaît.

v Une commode-buffet, ou armoire de ce genre (comme on voudra l'appeler), en sapin, comme les caisses Dombasle, ne coûterait guère plus et durerait bien davantage.

»Pour établir économiquement un fruitier de cette espèce, voici ce qu'on devrait faire : on placerait de chaque côté de la pièce, des tasseaux, depuis le parquet jusqu'au plafond; on mettrait des supports à la distance de 80 centimètres ou même 1 mètre 60 , pour porter les traverses sur lesquelles on établirait les tiroirs. Il est entendu que la pièce devrait être fraîche, exempte d'humidité, saine et à l'abri de la gelée Il suffirait d'étiqueter chaque tiroir pour qu'à la minute on trouve les fruits demandés.

- Pour conserver les fruits, pommes, poires, raisins, il suffit de les placer dans ces caisses. Chaque caisse de la dimension adoptée par Mathieu de Dombasle ayant 80 centimètres de long, $̋ 5$ centimètres de large et 10 centimètres de profondeur, le tout mesuré intérieurement contenait 100 beurrés ou bon chrétien de belle taille. 
Quant à la conservation elle ne laisse rien à désirer, d'après l'illustre praticien.

- Les fruits, dit-il, se conservent facilement dans ces caisses, et cette bonne conservation est vraisemblablement due à la stagnation complète de l'air dans cet appareil. On s'efforce d'obtenir autant qu'on le peut cette condition dans les fruitiers ordinaires, parce qu'on a reconnu que c'est elle qui contribue le plus à la conservation des fruits; mais quelques soins que l'on prenne, il est impossible de l'atteindre dans le local le mieux clos, avec autant de perfection qu'on l'obtient sans aucun soin dans les caisses. On sent, toutefois, qu'il est encore plus indispensable ici que dans toute autre disposition de ne serrer les fruits dans les caisses que lorsqu'ils sont entièrement exempts d'humidité, puisqu'il ne peut plus s'y opérer d'évaporation. »

- Les principaux avantages que l'on trouvera dans l'emploi du fruitier portatif, ajoute-t-il, consistent non-seulement dans la possibilité de loger une trèsgrande quantité de fruits dans un très-petit espace, et de les tenir à l'abri des animaux malfaisants ; mais aussi dans la facilité avec laquelle se fait lè service pour soigner et trier les fruits en enlevant ceux qui viendraient à se gâter ou dont on a besoin pour la consommation journalière.

a Chaque caisse, dans les dimensions que je viens d'indiquer, dit en terminant Mathieu de Dombasle, coûtera de 7 š centimes à 1 franc, selon que le prix du bois sera plus ou moins élevé dans la localité et que la fabrication sera plus ou moins soignée. ' 
On voit que, comme toujours, il prévoit tout et se rend compte de tout. Nos tiroirs, faits en peuplier, ne coûteraient pas davantage, peut-être moins; mais ils ne dureraient pas autant que le sapin si le fruitier avait un peu d'humidité. L'emploi du peuplier ne serait économique que dans une pièce parfaitement saine.

Quel que soit le mode de conservation qu'on adopte, il faut :

$1^{\circ}$ Que les fruits soient récoltés à point, comme nous l'avons dit à l'article Cueillette;

$2^{\circ}$ Que la cueillette soit faite par un beau temps;

$3^{\circ}$ Que les fruits soient rentrés secs et qu'ils restent étalés sur de la paille, pendant quinze jours au moins, dans un grenier exposé au nord, bien aéré jour et nuit, pour les faire sécher avant de les loger ;

$4^{\circ} Q u^{\prime} u n e$ fois serrés, ils soient surveillés fréquemment pour enlever les pourris, meurtris ou tảchés, qui feraient gâter ceux qui sont sains.

COMMERGE DES FRUITS

Nous avons déjà dit que les fruits pouvaient donner lieu à un commerce très-lucratif; pour appuyer notre opinion, nous croyons utile d'emprunter à l'excellent ouvrage de M. P. de Mortillet les lignes suivantes, établissant la moyenne du prix des poires sur la Halle de Paris aux différentes époques de l'année. 
“Les poires, dit-il, valent à Paris suivant qualité:

En

juillet et aoùt, le cent, de $3 \mathrm{fr}$. à $2 \% \mathrm{fr}$. septembre et octobre, - $3 \mathrm{fr}$. à $40 \mathrm{fr}$. novembre et décembre, _ $\quad 3 \mathrm{fr}$. à $60 \mathrm{fr}$. janvier et février, $\quad-\quad 5 \mathrm{fr}$. à $80 \mathrm{fr}$. mars et avril, _ $\quad 5$ fr. à $100 \mathrm{fr}$.

"Ce tableau représente la moyenne des prix extrêmes des quatre dernières années. „) (185\%6, 18577, 1858, 185ั9.) “Ce qui frappe à la première inspection, c'est l'écart qui existe entre le prix des fruits de qualité inférieure et celui des fruits de premier choix, tandis que le prix des premiers ne varie pour ainsi dire pas, quelle que soit la saison; les seconds arrivent à valoir 1 franc la pièce. »

Nous ajouterons que depuis la publication du livre de M. de Mortillet, le prix de bonnes poires a encore augmenté. Il n'est pas rare de les voir vendre au mois de mai 1 fr. $2 \breve{3}$ et 1 fr. 50 cent. la pièce ; ce qui prouve que les fruits de longue garde et de bonne qualité offrent infiniment plus d'avantage que les fruits médiocres.

\section{EMBALAGE DES FRUITS}

L'emballage des fruits en général, et de la poire en particulier, exige des soins d'autant plus minu-. tieux que le prix en est plus élevé. Il y a donc plusieurs modes d'emballer; les expéditeurs en em- 
ploient trois : le premier pour les fruits communs, le second pour les beaux fruits et le troisième pour les fruits supérieurs.

Pour les fruits communs, on prend des paniers carrés de 40 centimètres de profondeur sur 50 centimètres de large et 70 de long. On garnit de paille le fond et les côtés; puis on étend une couche de regain sur la paille et on y place un rang de fruits; on met une autre couche de regain et un autre lit de fruits, et ainsi de suite jusqu'à ce que le panier soit plein. Arrivé en haut, on met une couche de regain qu'on recouvre d'un lit de paille et on ferme en forçant le couvercle dessus pour empêcher le ballottement.

Pour les fruits de seconde qualité, qui atteignent 20 francs le cent, il faut procéder avec un peu plus de soin. Au lieu de paniers, on emploie des caisses et on emballe comme nous venons de le dire; mais on augmente l'épaisseur des couches de regain et on serre fortement pour qu'il n'y ait pas de ballottement. Les caisses ne se déformant pas comme les paniers, les fruits restent absolument dans la position où on les met.

Pour les fruits extra qui dépassent 20 francs le cent, il faut employer tous les moyens possibles pour éviter les avaries de route; on procède comme il suit :

On prend des caisses de 30 centimètres de profondeur sur 50 centimètres de large et 70 de long. On met une couche de regain de foin de prairie naturelle et un peu de rognures de papier/1 centimètre 
environ d'épaisseur); puis on place un lit de fruits enveloppés d'une feuille de papier Joseph, d'abord, et d'une autre feuille de papier sans colle un peu plus fort. On glisse des rognures de papier entre chaque fruit en les pressant assez pour empêcher tout mouvement; on remet encore des rognures de papier, un second lit de fruits et ainsi de suite jusqu'à ce que la caisse soit pleine. On termine alors par une forte couche de regain qu'on place sur les rognures de papier qui se trouvent sur le dernier lit de fruits, et on ferme en forçant un peu le couvercle de la caisse.

DE LA DÉGÉNÉRESCENCE ET DE L'EXTINCTION DES VÉGÉTAUX EN GÉNÉRAL ET DES RAGES FRUITIÈRES EN PARTICULIER. NÉCESSITÉ DE LES REMPLACER

Depuis quelques années, on se préoccupe de nouveau d'une question ancienne et déjà jugée : de la dégénérescence et de l'extinction des végétaux.

Des hommes d'un certain mérite ont nié ces faits et se refusent à admettre l'extinction des individus de la race fruitière. Il y a là une erreur matérielle qui choque le bon sens et qu'il est important de relever dans l'intérêt des planteurs.

Croire qu'un arbre peut vivre éternellement, multiplié par la greffe ou par la bouture, c'est croire qu'il y a des exceptions en sa faveur dans la nature: c'est fermer les yeux à la lumière. 
Il n’y a aucun fait à invoquer pour démontrer la vie éternelle d'un arbre; il y en a des milliers contre.

A ce propos, nous avons soutenu une polémique dans la Revue de l'Horticulture, dirigée par M. Barral. Or, comme nous rejetons les anciennes variétés de poiriers devenues improductives, nous croyons devoir rapporter cette discussion ici pour justifier le choix que nous avons fait. Nous extrayons donc ces lignes textuellement.

... Nous avons lu avec étonnement, dans plusieurs publications, l'opinion émise par plusieurs écrivains sur la non-dégénération des végétaux. Il suffit de jeter les yeux sur la nature pour voir que tout se modifie, dégénère et s'éteint. Que sont devenus tous les animaux, tous les végétaux qui peuplaient le globe il y a des milliers d'années?

Si l'on se donne la peine de lire les écrits de nos ancêtres, de ces savants profonds comme on n'en voit presque plus, on est bien vite convaincu que les végétaux subissent la loi commune et que les individus, végétaux ou animaux, ne sont pas persistants; ils dégénèrent et meurent: rien ne saurait vivre toujours, rien n'est éternel.

Parler de périodes de 20 ou de 25 ans, pour prouver la non-dégénérescence d'un végétal, c'est vouloir élever une montagne avec un grain de sable.

Ouvrons une petite brochure, un ouvrage spécial sur la question : De la dégénération et de l'extinction des variétés de végétaux propagés par les greffes, boutures, tubercules, etc., par Puvis, et voyons ce qu'en 
dit ce maître dont l'autorité ne saurait ètre contestée.

"..... Mais tous ces moyens (marcottes, boutures et greffes) de propager un individu par sa tige ou ses racines ne sont, en quelque sorte, que le morcellement ou la division de l'individu primitif, et ces parties, quoique séparées, lui appartiennent encore; c'est toujours une portion du même être, tige et racine dans les drageons, branche à laquelle on fait pousser des racines, dans les marcottes ou boutures, bourgeons ou boutons qu'on place sur des tiges, sur des racines ou sur d'autres sujets dans les greffes. Tous ces moyens de propagation ne sont donc que la continuation de la vie d'un même individu; c'est donc toujours, comme nous le disions dans un mémoire publié en 1817, une branche, un bouton ou une racine de l'individu primitif.....»

(.... Mais la mort est attachée à tous les individus matériels; elle est leur destinée dernière plus ou moins reculée, suivant les vues de la nature : la variété propagée par les soins de l'homme est donc destinée à périr comme l'individu primitif auquel elle est due et comme tous les êtres matériels; c'est une vérité d'analogie qui n'aurait peutêtre pas besoin de preuves. Toutefois nous croyons devoir l'appuyer sur un très-grand nombre de faits.....

"..... Nous voyons tous les jours, sous nos yeux, la mort frapper les végétaux qui nous entourent; les espèces annuelles semblent n'exister que pour fleurir, porter leurs graines et mourir, les espèces vivaces passent successivement par toutes les pé- 
riodes de jeunesse, de vigueur, d'âge mûr et de décrépitude.....»

«..... La longévité de quelques arbres ne prouve pas davantage la durée indéfinie de leur existence, quelque longue que soit cette durée, elle n'est, en quelque sorte, qu'un point dans la suite des temps; mais, en outre, il est probable qu'à voir de plus près les circonstances de ces arbres extraordinaires cette si grande antiquité se réduirait beaucoup, comme on a vu, par un travail 'éclairé, se réduire celle du zodiaque de Denderah.

( ... Au milieu d'organisations que nous voyons toutes finir comme nous-mêmes, quand le monde est couvert de débris des anciennes existences, que nous n'en voyons aucune survivre, comment serait-il possible d'admettre que la vie végétale fût une exception et qu'elle pût durer indéfiniment? Quand le monde lui-même, où nous sommes avec tous les ètres qui l'animent, doit prendre une fin ou passer à une autre forme; quand nous voyons que les êtres qui l'ont successivement couvert ont tous péri à diverses reprises, comment concevoir que ceux de la période où nous nous trouvons puissent avoir une autre destinée?...

„L'Lomme, par ses moyens artificiels, par les greffes, boutures ou marcottes, peut bien prolonger cette existence individuelle dans quelques espèces; il renouvelle en quelque sorte la jeunesse de la variété qu'il propage ; mais, à chaque renouvellement, cette jeunesse devient plus courte, la vie diminue d'étendue, la vigueur s'amoindrit dans ces existences 
dues à son industrie; mais l'inévitable mort attend toujours sa proie, et au bout d'un certain nombre de générations, la variété propagée finit comme le premier individu qui lui a donné naissance.

" La plupart des auteurs agronomiques admettent l'opinion que la propagation par boutures, marcottes, tubercules, tend à la dégénération....

„Pline nomme, sans les reconnaître, dans la culture de son temps, les variétés de fruits et de raisins décrites par Caton. Columelle se plaint que les vignes aminées qui, du temps de Caton, donnaient de grands produits en excellents vins, étaient dégénérées et devenues presque stériles. Ollivier de Serres qui, le premier d'entre les modernes, a écrit un bon ouvrage d'agriculture, recherche les variétés de Pline et de Palladius sans pouvoir les retrouver; si de là nous passons aux variétés que donne Ollivier de Serres lui-même, à peine retrouvons-nous un cinquième de celles qu'il nomme. Enfin, la moitié de celles que désigne La Quintinie ne sont plus connues; une partie peut bien avoir été négligée, mais un grand nombre a sans doute péri. »

(Ici Puvis cite des faits que nous croyons inutile de rapporter. Ils portent sur les grands poiriers de la Bresse qui ont en partie cessé d'exister ou qui ne sont plus que des arbres de faible dimension; sur la reinette des Gravelles qui est un arbre usé; sur les blanquettes qui périssent après quclques années; enfin sur la calville blanche.)

" La calville blanche, dit-il, la meilleure peutêtre des pommes, au lieu de s'élever aux dimensions 
qu'on lui voit encore dans les vieux individus, se chancre dans presque tous les terrains, donne encore de bons produits, mais le plus souvent sur des arbres petits et faibles, qui sont loin de la taille de leurs devanciers. »

Il cite aussi l'épargne, les beurrés gris, qui ne donnent que des arbres petits, délicats et maladifs, le beurré blanc, la reinette franche. Il ajoute : “...Ces espèces languissantes et faibles dans de grands arbres, retrouvent un peu de vigueur, sont encore fécondes et restent presque exemptes des maladies de leur vieillesse, si on les cultive en arbres nains dont une taille annuelle rajeunit le bois ; ainsi, particulièrement la poinme d'Api, la calville blanche et les beurrés sont féconds et montrent encore quelque vigueur quand on les cultive en arbres nains.

" Les Anglais ont fait aussi à ce sujet des observations très-précises et qui datent depuis longtemps; Marschall rapporte que dans plusieurs contrées certaines espèces de pommiers et de poiriers, qui jadis donnaient une très-grande abondance de fruits trèsbons, en donnent désormais très-peu ; que les arbres qu'on greffe de ces espèces ne donnent que des individus petits et faibles, rongés de chancres et de maladies, pendant que ceux dont on tire les greffes sont encore des arbres énormes, mais sur leur déclin; et dans toutes ces contrées, l'opinion est admise que les variétés transmises par la greffe s'éteignent peu à peu; cette opinion était donc devenue populaire avant d'ètre arloptée et propagée par les écrivains agronomiques. 
D ..... Knight, le même rlont on connaît de nombreuses et intéressantes expériences sur la végétation, a fait de cette opinion la base de travaux trèsremarquables.....

- Humphry-Davy, qui a appliqué avec une grande hauteur de vues la science à la pratique agricole, regarde ces faits de l'affaiblissement et de la mort des variétés comme non contestables.

D.... C'est par cette raison (que la greffe ne fait que continuer lindividu), que tant de variétés de pommes renommées autrefois par leur goùt et l'excellent cidre qu'elles donnaient se sont à peu près détériorées et menacent de disparaître tout à fait. Le golden pippin, la calville rouge et la moil, si parfaits dans le commencement du siècle dernier, ont atteint le terme extrême de leur détérioration : on a beau chercher à les maintenir par des greffes choisies, on ne fait que multiplier des variétés maladives et épuisées.

„Le raisonnement, l'analogie, les faits que nous voyons sous nos propres yeux, l'expérience de pays entiers, l'histoire du passé et les autorités agronomiques les plus graves, semblent done toutes d'accord pour prouver, comme une vérité, qu'il n'est plus possible de contester, que les variétés d'arbres à fruits qui, en résumé, prises séparément, se réduisent toutes à une seule individualité, périssent et s'éteignent comme toutes les existences matérielles.

\.... Nous avons vu que Columelle se plaignait de la disparition des variétés cultivées du temps de Caton; plus tard, Palladius annonçait l'affaiblissement de variétés plus récentes; il y aurait dlonc entrè 
ces auteurs une durée de génération de ces variétés. Treize siècles après, Ollivier de Serres ne retrouvait plus aucune des dernières variétés décrites; sans doute, plusieurs générations de variétés nouvelles, dans tout cet espace de temps, avaient achevé leur carrière. La Quintinie, qui a paru un siècle et demi après Ollivier de Serres, ne nous donne pas la moitié des variétés que nomme ce dernier. Duhamel ne retrouve plus qu'une partie de celles de la Quintinie. Le bon chrétien d'été qui, sous Louis XIII, lors de son introduction en France par saint Vincent de Paul, était un fruit excellent et très-fécond, et par conséquent appartenait à un arbre en pleine vigueur, est devenu un fruit taché, pierreux, peu abondant, et les jeunes arbres qui le portent sont malingres et faibles, signes de dépérissement qui annoncent sa fin. Enfin, les Anglais attribuent à un jardinier d'Henri VIII une partie de leurs meilleures variétés qu'ils voient maintenant s'éteindre sous leurs propres yeux, en donnant peu de fruits et des fruits médiocres portés par des arbres sans vigueur, et qui, dès leur jeunesse, donnent des signes de décrépitude.

»De toutes ces diverses observations nous devons conclure que la durée des variétés propagées par la greffe serait de plusieurs siècles; mais cette durée est bien loin d'être uniforme : il est de beaux fruits pour lesquels elle est évidemment plus courte. Lorsque Duhamel écrivait, l'arbre qui été la souche du bézy-chaumontel existait encore en Normandie dans la forêt de Chaumontel; depuis ce temps, cette 
variété ne peut plus s'obtenir en grand arbre; elle donne quelques fruits rares sur des arbres nains qui périssent eux-mêmes bientôt en perdant leur bois et après avoir jeté quelques bourgeons sans vigueur : ce fruit très-perfectionné, mais peu ancien, semble donc déjà toucher à sa fin... »

Les lignes qui précèdent étaient une réponse faite à des opinions émises un peu partout. M. Palmer se les attribua et fit diverses objections auxquelles nous avons dủ répondre ce qui suit :

II. Palmer dit : ‘ Ce qui nous intéresse..., ce n'est pas de savoir si les plantes propagées de boutures sont périssables, mais si elles le sont plus que celles propagées de graines; là est toute la question. »

Les observateurs et les praticiens disent oui; je dis oui et M. Palmer lui-même dit oui sans le vouloir. En effet, voici sa réponse : a Il est évident que si vous plantez une bouture malade, malingre ou affaiblie : maladive, malingre et faible sera la plante produite. , M. Palmer admet donc la dégénérescence forcément. Du reste, il ajoute : ‘... Mais qu'une bouture saine mise dans la même terre, en mème temps et dans les mêmes conditions qu'une graine, doit, etc... ๖ Admettre qu'une bouture doit être saine, c'est admettre qu'elle peut ne l'être pas. II. Palmer confirme donc encore la possibilité de la dégénérescence. D'ailleurs, alors même que la bouture serait saine au moment où elle est détachée de l'arbre, n'a-t-elle pas toute une série d'accidents à redouter? La sècheresse, le froid, la pluie, les coups de soleil, l'infertilité du sol, etc., ne sont-ils pas 
autant de causes qui peuvent la détériorer et l'anéantir, causes qui n'ont presque aucune influence sur la graine?

La comparaison ne saurait être exacte. La graine n'a que de faibles besoins à satisfaire graduellement au fur et à mesure que ses organes se développent; la plante-bouture privée de tout ne résiste à ce sevrage de la plante-mère qu'à la condition de souffrances et de langueurs plus ou moins prolongées qui l'altèrent et influent sur la durée de son existence. La bouture se trouvant ainsi dans-des conditions anormales, emprunte beaucoup au sol et sa prospérité dépend de l'état où elle le trouve; bon elle prospère, mauvais elle végète misérablement, obligée qu'elle est de vivre pour ainsi dire de sa propre substance. Dans le premier cas, la graine germe et réussit, et l'individu né est assez fortement constitué pour vivre; dans le second, elle ne germe pas.

M. Palmer ajoute : “ Mais, au point de vue pratique, la question ne peut avoir d'autre but que de chercher à faire abandonner l'usage de la bouture et de la greffe. 》 Étrange raisonnement! J'ai cité des faits signalés, étudiés et reconnus par nos anciens physiologistes, rien de plus. La conclusion à en tirer est celle-ci : il faut s'attendre à voir disparaître toutes les anciennes variétés; il est temps de pourvoir à leur remplacement; mais il reste entendu que la greffe et le bouturage étant les seuls moyens de multiplication, il faut les employer tant qu'il sera profitable de le faire.

" Puis il faudrait bien prouver que, dans tous les 
cas cités, dit II. Palmer, les fruits propagés de boutures et de greffes sont bién et dûment éteints ou dégénérés. " A moins de ne pas vouloir ouvrir lès yeux à la luinière, on peut en voir les preuves partout. Je crois en avoir cité un assez grand nombre pour ne pas être obligé d'y revenir. A moins de supposer que nos anciens physiologistes n'étaient que des ignorants ou des écrivains de mauvaise foi, il faut bien accepter ce qu'ils en disent.

Si l'on révoque en doute les écrits des Columelle, des Palladius, des Ollivier de Serres, des La Quintinie, des Duhamel, des Decandolle, des Puvis, etc., on peut tout aussi bien révoquer en doute toute l'histoire.

S'il avait eu des raisons péremptoires à donner II. Palmer aurait combattu et détruit d'une manière précișe toutes les assertions des hommes illustres que je viens de citer; car, jusque-là, elles continueront de faire foi, parce qu'il n'est à la connaissance de personne qu'elles aient été contredites sérieusement.

"Quiconque veut se donner la peine de prendre le chemin de fer de Saint-Malo, ajoute M. Palmer, et de visiter l'île de Jersey, pourra s'assurer de visu et de gusto que les poires Chaumontel ne sont pas partout dégénérées ou éteintes, comme le pensait Duhamel. » Je ne suivrai pas M. Palmer à l'île de Jersey pour lui démontrer que le Bési-Chaumontel est tellement dégénéré qu’il sera bientòt supprimé complétement là où il ne l'est pas encore. M. Palmer n'a qu'à qu'à visiter les vergers du centre de la France pour se convaincre que sa culture est devenue im- 
possible : ce n'est plus qu'un pauvre arbuste qui ne donne plus que de pauvres fruits, comme le doyenné d'hiver, le bon chrétien d'hiver, le Saint-Germain, etc. Que sont devenues ces belles variétés, ces arbres gigantesques qui produisaient des fruits si succulents, si délicieux? De tristes avortons qui s'étalent tristement le long des murs, comme des phthysiques au soleil d'avril!

“ On n'a pas non plus ouï dire, je crois, que les saules, les osiers, propagés de plancons depuis les temps les plus reculés, aient perdu de leurs qualités ou fussent éteints, ajoute M. Palmer. »

M. Palmer oublie que les saules et les osiers se régénèrent constamment de graines. Ils auraient pu s'éteindre bien des fois sans qu'on fût à même de s'en apercevoir. Cependant voici un fait qui dénoterait qu'ils ont dégénéré :

Il y a trente à trente-cinq ans, on a extrait d'une ancienne tourbière des saules qui y avaient été renversés, soit par l'orage. soit pour exhausser ou combler le sol. Ils étaient d'une dimension colossale : quelques-uns mesuraient 26 à 28 mètres de longueur, et à l'extrémité supérieure, ils avaient 35 à 50 centimètres de circonférence, ce qui fait supposer qu'ils pouvaient atteindre de 35 à 40 mètres de hauteur totale. Je crois qu'il serait impossible d'en troụver aujourd'hui d'une telle dimension.

A la même époque à peu près, jai vu abattre dix ou douze peupliers d'Italie; les plus faibles mesuraient 3 mètres de circonférence; deux dépassaient 4 mètres à 1 mètre du sol. C'est de ces types que 
sont sortis les milliers de peupliers qui peuplaient, il y a vingt ans, les vastes prairies de l'arrondissement de Châtillon-sur-Seine. Eh bien! les plus gros que j'aie vus depuis n'avaient pas plus de 1 mètre こ0 centimètres de circonférence, et ils étaient couronnés (morts en tête), ce qui dénote que leur existence était arrivée à son terme.

Le paysan qui, dans ce cas-ci, est un bon observateur, dit tout nettement que le peuplier d'Italie est dégénéré. Le peuplier suisse, au contraire, ayant pu se resemer, a une grande vigueur, une grande supériorité sur lui : cependant, dans le principe, il lui était très-inférieur et produisait beaucoup moins de bois.

- Pour la vigne même, dit encore M. Palmer, ce produit si essentiellement français, et dont l'histoire est par conséquent bien connue en France, peut-on remonter à l'origine et réellement prouver que telle ou telle espèce ait dégénéré ou soit morte de vieillesse? je ne dis pas mise au rebut pour faire place à de meilleures espèces, mais morte de sa belle mort."

A moins de taxer de mensonge ou d'ignorance, comme je le disais tout à l'heure, les anciens auteurs qui étaient des observateurs aussi sagaces, des historiens aussi fidèles que l'on pourrait l'être de nos jours, de nombreuses variétés de vignes auraient disparu. Voici ce qu'en dit Puvis : . ..... Ainsi, les vignes aminées qui, sous Caton, montraient beaucoup de vigueur et donnaient le vin le meilleur et le plus abondant, sous Columelle avaient perdu toutes leurs qualités, étaient devenues faibles, peu productives et périssaient promptement. " Il n'est point ques- 
tion ici de vignes ni vues ni connues, le fait est précis. De ce que nous n'avons ni vu, ni connu ces vignes et beaucoup d autres, est-ce une raison pour qu'elles n'aient pas existé ? Par cette même raison, on pourraitnier l'existence de Charlemagne et de Henri IV.

$\mathrm{Au}$ surplus, il est bien inutile de démontrer que certaines vignes sont mortes de leur belle mort. Pour celui qui les cultive, elles sont mortes le jour où elles cessent de produire et de lui être profitables ; autrement, il faudrait admettre qu'il eùt assez de patience pour attendre leur fin, quoi qu'il lui en coûte. Ce serait trop.

En compulsant les anciens auteurs, on trouverait des exemples très-nombreux de ces disparitions; mais, indépendamment de ce qu'un tel travail ne saurait rentrer dans le cadre d'un journal comme la Revue, je ne me sens ni le désir, ni le courage, ni le temps de le faire. Quelques témoignages contemporains suffiront.

Dans certains vignobles du centre de la France, où l'on cultive la vigne en perchées à longue taille, au dire des anciens vignerons du siècle dernier, on laissait jusqu'à douze ou quatorze broches à chaque branche coursonne, et chaque branche avait de dix à douze mètres de longueur. Aujourd'hui, j'ai revu ces vignes : les coursonnes n'ont plus que de quatre à six mètres de long, et le nombre des broches est diminué de moitié, non-seulement là où les vignes sont cultivées depuis longtemps et le sol usé, mais dans les terres vierges.

Tous les semeurs de vigne savent qu'elles ne pro- 
duisent, dans les dix ou douze ans qui suivent les semis, qu'à la condition de leur laisser pousser tout le bois possible; tandis que les boutures et marcottes prises sur le pied-mère se mettent à fruit beaucoup plus vite et poussent moins de bois. La conséquence est facile à tirer.

J'ai vu, et il y a quelques années il existait encore, un cep de chasselas, dit de Fontainebleau, bien que cette vigne soit née bourguignonne, mesurant quatrevingts centimètres de circonférence et portant environ deux cents mètres debranches coursonnes.Ce cep, tout le monde a pu le voir, comme moi au jardin du PetitVersailles, à Châtillon-sur-Seine (Côte-d'Or). Je l'ai signalé dans une brochure, il y a vingt-six à vingthuit ans. Trouverait-on aujourd'hui un cep qui promette d'arriver à cette taille? Citez-moi un châtaignier greffé qui ait vécu ce qu'a vécu le châtaignier de Montélimar; des cornouilliers greffés de la force et de l'âge de ceux qui bordaient les propriétés des Montmorency et leur servaient de limites. Les titres de famille les signalaient comme tels, et ils remontaient à deux siècles et plus. Trouvez-moi un pommier, un cerisier, un merisier, greffés ou de marcottes, qui atteignent la dimension de ceux qui servent de pieds corniers dans les forêts de l'État ou autres. Leurs enfants sont morts et eux vivent encore.

Le type vit souvent des siècles et presque autant que tous les sujets greffés ou bouturés de lui.

La greffe et la bouture peuvent prolonger l'existence d'une variété; mais elles ne sauraient la prolonger indéfiniment, et chaque fois quon bouture 
ou greffe un arbre, on descend un échelon de l'échelle qui la conduit à la mort.

M. Palmer termine en disant : a Ma raison se refuse à admettre l'hypothèse de la continuation d'un individu par le bouturage et de la non-individualité de la plante NÉE de bouture. ")

II. Palmer dit une plante NÉE de bouture. Je ne puis laisser passer cette expression vicieuse; car si j'admettais qu'une plante naît de bouture, j'admettrais le renversement de tout mon raisonnement: ce serait la négation de toutes les citations ci-dessus. Si la bouture n'est pas la continuation de l'individu, elle en diffère donc. Or, je demande en quoi? Si la bouture diffère de l'individu, il y a dégénérescence. Si la bouture ne diffère pas de l'individu, c'en est done la continuation, et il n'y a pas d'individualité. Si la raison de M. Palmer se refuse à croire à la non-individualité, la mienne se refuse bien plus à croire à̀ l'individualité qui n'en est pas une.....

Quand j'interroge des yeux ces vieilles variétés d'arbres fruitiers, si belles, si vigoureuses, si fertiles, si robustes, qu'on rencontrait dans tous les sols, à toutes les expositions, qui produisaient des fruits si beaux, si succulents, si nombreux ; quand je vois ces variétés, aujourd'hui réduites à l'état de nains dont les squelettes desséchés ont besoin d'être rognés, ravalés, déchiquetés tous les printemps, pour qu'ils puissent pousser quelques misérables rameaux; quand je vois ces fruits difformes, disparates, tavelés, gersés, chancreux, dont la plupart 
tombent et fanent avant la maturité; quand je déguste ces fruits et que je les sens croquer comme de la pierre sous la dent, que mon palais attend en vain cette eau abondante, fraîche, douce, parfumée qui les distinguait jadis, je m'écrie avec le poëte :

.... Eheu! quantum mutatus ab illo!

Ajoutons quelques autres réflexions, car le sujet est inépuisable; il suffit de lire dans le grand livre de la nature pour y trouver la confirmation de ce fait que tout naît, vit et meurt, chez les végétaux comme chez les animaux.

Que sont devenues ces prêles qui atteignaient jusqu'à vingt-cinq mètres de hauteur et qui ne dépassent pas soixante centimètres, aujourd'hui? Les fougères qui, de dix mètres de hauteur, sont descendues à un mètre cinquante? Il y a des milliers de plantes qui ont suivi la même marche. Qu'y a-t-il donc d'étonnant que les arbres fruitiers aient subri le même sort?

Citons aussi l'opinion de Boitard, ce savant dont la cendre est encore toute chaude.

.... « Dans les plantes ligneuses, la mort de vieillesse est plus difficile à expliquer; aussi quelques botanistes célèbres la nient-ils; je ne puis être de leur avis.

.... Mon hypothèse fait concevoir la mort naturelle : on n'est plus forcé d'admettre l'opinion ahsurde qu'un être a commencé pour n'avoir pas de fin. ..... a La bouture est une partie de la plante qui s'en sépare pour former un être distinct, mais animé 
par la même force vitale, et ne formant, pour ainsi dire, qu'une continuation du végétal qui l'a produite. Les boutures, au nombre desquelles se trouvent naturellement placées la greffe et la marcotte, n'étant qu'une continuation du même être le reproduisent avec toutes les particularités qui lui sont propres, c'est-à-dire qu'elles redonnent jusqu'aux moindres variétés. »

Ceci explique pourquoi nous avons rejeté les anciennes variétés de poiriers. Il est donc tout à fait déraisonnable de lutter contre l'évidence des faits et de s'entêter à vouloir planter des arbres qui n'offrent plus d'intérêt à la culture et qui payent si mal les soins qu'on leur donne. Il faut y renoncer. Ce qui nous reste à faire, c'est de choisir parmi les variétés nouvelles celles qui sont les plus méritantes et qui pourront, par leurs qualités, leur vigueur, leur fertilité, nous faire oublier les anciennes. Les semis ne doivent pas être non plus négligés ; mais ce qu'il est important de faire, c'est de n'admettre que des fruits de bonne qualité et de garde. Plus de 13 ou 1,400 variétés de poires sont aujourd'hui dans le commerce. C'est à peine s'il y en a 50 qui doivent être cultivées ; car toutes les autres peuvent être avantageusement remplacées par celles-ci. Avis aux amateurs et aux planteurs. 


\section{DEUXIÈME PARTIE}

\section{CONSIDÉRATIONS GÉNÉRALES SUR LA VÉGÉTATION}

ET NOTIONS DE PHYSIOLOGIE VÉGÉTALE

Avant de traiter de la taille, il serait utile de s'étendre longuement sur les principes de physiologie végétale pour que l'on puisse raisonner toutes les opérations qu'elle comporte; malheureusement, ce serait entreprendre un travail au-dessus de nos forces. La théorie a été 'négligée dans tous les ouvrages, même spéciaux, qui traitent de la taille des arbres fruitiers; la plupart des auteurs modernes n'ont fait qu'effleurer cette question très-ardue pour tous, et surtout pour celui qui l'étudie. Un seul ouvrage spécial de quelque importance a été publié, c'est le Manuel de physiologie végétale, par Boitard ${ }^{1}$;

1. Chez Roret, libraire, rue Hautefeuille, 12, à Paris. 1 vol. avec planches, $3 \mathrm{fr}$. 
aussi y renvoyons-nous le lecteur qui voudra en faire une étude sérieuse. Quant à nous, nous nous bornerons à suivre à peu près les errements de nos levanciers, c'est-à-dire à donner les principes de la taille, sans nous arrêter aux nombreux détails théoriques qui sont du domaine de la science, nous en tenant purement et simplement aux opérations pratiques et aux démonstrations générales, mais en indiquant des moyens nouveaux de dresser, diriger et gouverner les espaliers.

Nous ne feron: pas de la science; ce n'est pas notre affaire, ici surtout. Nous décrirons le plus rapidement possible les principes de la taille, et nous chercherons à prouver que les auteurs modernes se sont égarés en prônant des formes qui ne sont ni rationnelles, ni productives, ni économiques, ni même possibles.

Nous envisageons la culture des arbres fruitiers comme une affaire de production et non de luxe. C'est pourquoi nous n'admettons aucune des formes nouvelles qui sont plus ou moins destinées à exercer la patience de l'arboriculteur et à orner les murs. Nous croyons que le meilleur ornement qu'on puisse faire, c'est de produire beaucoup de fruits, d'avoir des arbres faciles à gouverner, vigoureux, de longue durée et de débarrasser l'arboriculteur deces soins interminables et minutieux pris en pure perte. 
DE LA TAILLE - DE QUOI SE COMPOSE UN ARBRE

Un arbre se compose :

\section{De racines;}

$2^{\circ}$ D'une tige ou tronc ;

$3^{\circ}$ De branches;

$4^{\circ}$ De feuilles ;

วั0 De boutons ou yeux.

Nous négligeons tout le reste comme s'éloignant de notre plan. Il suffira de connaître les fonctions de ces cinq parties pour tailler convenablement; mais il est indispensable de se bien pénétrer du jeu de ces différents organes si l'on veut comprendre et raisonner les opérations que nécessite le gouvernement d'un arbre.

10 Les RAcines servent à fixer l'arbre au sol et à lui transmettre la nourriture qu'elles y puisent. Il y en a de deux sortes : les racines pivotantes qui se dirigent verticalement vers le centre de la terre, les traçantes qui sont horizontales ou qui se ramifient à la surface du sol. Les racines àbsorbent les sucs nourriciers au moyen de petites bouches aspirantes nommées spongioles qui sont situées à l'extrémité du chevelu, c'est-à-dire de toutes les petites racines. Done, plus un arbre a de chevelu, plus il a de bouches absorbantes pour lui procurer de la nourriture.

Quand on plante un arbre, on doit respecter tout ce chevelu comme étant la partie essentielle à la 
reprise. Il ne faut retrancher que l'extrémité des racines meurtries ou cassées, et ne jamais toucher sous aucun prétexte aux parties saines.

2 La Tige ou Le tronc. - On distingue dans la tige ou le tronc, ainsi que dans toutes les branches qui en sont le prolongement, l'écorce, le liber, le bois, le ligneux et la moëlle.

La tige part des racines ou du collet qui est le point de jonction de ces deux parties, pour s'élever au-dessus du sol.

L'écorce se compose de l'enveloppe pour ainsi dire herbacée qui recouvre les couches corticales et le liber qu'on rencontre avant d'atteindre le bois.

Le liber est la couche qui est placée entre l'écorce et le bois ; tous les ans il se transforme en ligneux ou aubier, et celui-ci en bois.

Le bois occupe toute la partie qui se trouve entre l'aubier et la moëlle : c'est par ses pores que la sève circule, tant que les vaisseaux sont assez développés et libres, c'est-à-dire tant que l'arbre est jeune et bien portant; mais par l’âge, ils s'obstruent, se rétrécissent, la circulation de la sève se ralentit, puis s'arrête et l'ärbre meurt.

$\boldsymbol{L} a$ moëlle est une substance légère et sèche qu'on trouve au centre de l'arbre, dans l'étui médullaire et dont les fonctions sont complétement inconnues : tout ce qu'on en a dit jusqu'à présent ayant été controversé.

$3^{\circ}$ Les branches, ne sont autre chose que le prolongement du tronc. Elles sont de deux sortes: lesbranches à bois, ou branches de charpente, destinées 
à développer l'arbre et à donner naissance à d'autres branches plus petites qu'on nomme brindilles ou branches à fruit. On donne le nom de rameau, aux branches d'un an, munies de boutons ou yeux à bois.

$4^{0}$ Les feuilLes sortent des boutons, gemmes ou yeux; elles sont composées de trois parties bien distinctes, le pétiole, les nervures et le limbe. La face supérieure est lisse, comme vernissée, tandis que la face inférieure est, le plus souvent, recouverte de poils et criblée de petits trous (stomates), par lesquels l'arbre absorbe la nourriture contenue dans les fluides de l'air, notamment l'acide carbonique. Ce phénomène a lieu pendant la journée; l'air s'introduit, chargé des gaz qu'il contient, dans les pores des feuilles où le contact de la lumière le décompose, fixe le carbone et dégage l'oxygène. Pendant la nuit le contraire a lieu : elles s'emparent de l'oxygène et dégagent de l'acide carbonique.

On voit que les arbres se nourrissent, respirent et transpirent par les feuilles : elles ont donc une grande importance ; aussi doit-on les ménager et ne jamais les supprimer sans nécessité.

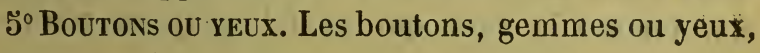
renferment les rudiments des feuilles, des fleurs et des branches. Ils sont entourés d'écailles sèches et recouverts d'une sorte de vernis ou enduit glutineux qui est destiné à les préserver pendant l'hiver contre les intempéries. Ces enveloppes sont d'autant plus épaisses que le climat est plus froid; ainsi les arbres du nord les ont beaucoup plus épaisses que ceux des pays chauds: 
Il y a deux sortes d'ail ou bouton: le bouton à bois et le bouton à fruit. Le premier produit du bois et le second du fruit.

Le bouton à bois est toujours pointu, il affecte la forme d'un $\mathrm{V}$ renversé $\boldsymbol{\Lambda}$; le bouton à fruit, au contraire, est toujours gros, court, arrondi; il a li forme d'un $\mathbf{U}$ renversé $\mathbf{\Omega}$.

Boutons ou yeux à bois. - Il y en a de quatre sortes : les latéraux, les terminaux, les latents et les adventifs.

Les yeux latéraux sont ceux qui naissent le long des rameaux, et les terminaux ceux qui se trouvent. à l'extrémité; ils sont plus gros que les premiers. Les yeux latents sont placés sur le vieux bois; ils restent souvent plusieurs années sans se développer si une circonstance favorable ne les oblige pas à le faire : souvent même ils s'éteignent complétement et disparaissent. Les yeux adventifs sont imperceptibles; ils percent à travers l'écorce du vieux bois, sans que rien ne fasse supposer qu'ils existent. Ces yeux ne sortent qu'à la suite d'une forte amputation, de la mort d'une grosse branche ou de toute autre cause qui refoule la sève qui se fait jour en rompant l'écorce.

Les boutons ou yeux à fruits, sont les téguments ou enveloppes des fleurs; ils sont, comme les boutons ou yeux à bois, recouverts de sortes d'écailles, mais plus développés, plus ronds, ils végètent plus tôt au printemps.

Les boutons à fruit sont presque toujours placés à l'extrémité de petites branches qu'on nomme lam- 
bourdes ou brindilles, et dont la longueur peut varier de 3 à 2.0 centimètres dans les arbres à fruits à pépins. Quelquefois on les rencontre à l'extrémité des rameaux, mais c'est un cas exceptionnel.

EFFETS DES YEUX A BOIS ET DES BOUTONS A FRUIT SUR LA VÉGÉTATION

Les yeux à bois et les boutons à fruit, ont un effet complétement opposé sur la végétation de la branche où ils se trouvent. Si une branche contient beaucoup d'œils à bois elle aura une végétation d'autant plus belle que le nombre en sera plus grand. Si, au contraire, elle porte beąucoup de boutons à fruits, la sève, au lieu de produire du bois, produira des fruits et elle restera languissante.

Quand une branche ne porte que des yeux à bois, elle est susceptible de devenir très-vigoureuse ; aussi faut-il la tailler long si l'on veut beaucoup de bois, et court s'il est utile de n'avoir qu'une végétation modérée, et de faire passer la sève dans une autre partie de l'arbre.

La multiplicité des boutons à fruits sur une branche, en amène la ruine; on doit donc généralement tailler court les brindilles ou branches qui portent un grand nombre de ces boutons. 
DES PRODUCTIONS FRUITIERES

On donne le nom de productions fruitières à toutes les petites branches ou ramifications qui se trouvent sur les branches charpentières, telles sont les brindilles, les dards, les lambourdes, les bourses.

Il est essentiel de les distinguer et de les reconnaître pour opérer convenablement la taille.

La brindille est un petit rameau flexible, grêle, allongé, qui a de 10 à 25 centịmètres au plus de longueur; les yeux sont petits et généralement rapprochés. Elle se met promptement à fruit sur les arbres qui n'ont pas une grande vigueur.

Le dard est un rameau encore plus court que la brindille, il n'a que 1 à 10 centimètres au plus de longueur : il est terminé par un œil allongé ou conique, la première année ; mais les années suivantes il grossit et finit par devenir un bouton à fruit. ll lui faut ordinairement deux ou trois ans pour arriver à ce point; cependant quelquefois il se transforme dès la première année. On ne taille ni ne supprime les dards, à moins qu'ils soient en trop grand nombre sur le même point.

La lambourde n'est autre chose qu'un vieux dard touronné ou terminé par un bouton à fruit. Ce bouton est facile à reconnaître, il est gros, court, ef la lambourde est ridée au lieu d'être lisse comme le dard d'un an.

La bourse est la production du bouton à fruit, o'est 
le point où les fleurs et les fruits étaient attachés. C'est une partie renflée, charnue, tendre, portant plusieurs yeux qui se transforment en boutons à fruits; mais que l'on peut convertir en dards et même en branches de 40 à 60 centimètres de longueur.

DES BRANGHES ADVENTIGES

On donne le nom de branches adventices aux gourmands, aux bourgeons et faux rameaux qui naissent le long de la tige ou des branches charpentières, ou même sur les branches à bois et à fruit.

Le gourmand est un rameau ou une branche d'une grande vigueur qui s'empare de presque toute la sève de la branche qui lui a donné naissance. On le reconnaît à sa vigueur et à son empatement qui est très-large. Absorbant la sève à son profit, comme nous venons de le dire, il menace lexistence de toutes les autres parties de l'arbre. Les suppressions de grosses branches, la taille courte donnent lieu à la production des gourmands.

Les bourgeons et faix rameaux sont presque toujours nuisibles, comme le gourmand, et ont les mêrmes effets, quoiqu'à un degré infiniment moindre. On les rencontre sur les vieux arbres, sur ceux qui ont été taillés court, sur ceux dont la sève est entravée dans les branches charpentières, ou dont la tête a été brisée ou amputée. 
OPÉRATIONS PRATIQUÉES LORS DE LA TAILLE OU APRÉS QUELLE A ÉTÉ FAITE

L'ensemble des diverses opérations qui se pratiquent sur un arbre, constitue la taille, ou tout au moins rentre dans son cadre; car dans le gouvernement d'un arbre tout se lie, tout s'enchaîne.

Les opérations auxquelles on soumet les arbres sont assez nombreuses. Ce sont d'abord : la coupe, le rapprochement, le ravalement, le recépage, l'entaille, l'incision, l'ébourgeonnement, l'éborgnage, l'arqúre, le palissage, le pincement, la taille d'été, le cassement.

La coupe. - On donne le nom de coupe à l'opération qui consiste à enlever tout ou partie des rameaux soumis à la taille. Elle s'exécute à l'aide de la serpette et du sécateur. La serpette sert à tailler les branches de prolongement ou branches charpentières et le sécateur les brindilles et branches à fruit, parce que les plaies qu'il fait sur les grosses branches sont désagréables à voir et peuvent entraîner, non-seulement la perte de l'œil voisin de la coupe, ınais même celle de la branche taillée.

La coupe doit se faire à 4 ou 5 millimètres derrière l'œil pour les bois durs, et à 9 ou 10 pour les bois tendres. Plus le rameau est fort, plus la coupe doit être éloignée.

On doit donner un peu de pente à l'aire de la coupe, du côté opposé à l'œil, afin que la sève et l'eau ne s'écoulent pas sur l'œil. 
On nomme onglet le chicot qui reste entre l'œil et la coupe. Quand il est trop long on le rabat à la taille suivante.

En général il est préférable de laisser un petit onglet que de tailler trop près de l'œil, car alors il s'évente et ne pousse pas.

Pour couper bien net et bien sûrement, on prend le rameau d'une main, on place le pouce directement au-dessous de l'œil sur lequel on veut tailler et de l'autre main on fait jouer la serpette adroitement pour ne faire aucune blessure soit aux branches voisines, soit à soi-même.

Si l'on se sert du sécateur, il faut laisser un onglet plus long et on le raccourcit à la serpette pour que la plaie puisse se recouvrir. De même si l'on emploie la scie; mais, alors si la branche est grosse on recouvre la plaie avec du mastic mou ou mastic à greffer, pour éviter les gerçures, le dessèchement et l'infiltration de l'eau dans le canal médullaire.

Le rapprochement. - Rapprocher c'est tailler sur le vieux bois; c'est-à-dire au-dessous des rameaux de l'année précédente. On rapproche pour refouler la sève et faire percer des yeux destinés à regarnir les vides ou à obtenir des branches charpentières plus fortes. La taille alors se fait derrière un noeud, un coude, ou un pli de l'écorce, parce que c'est de là surtout que les yeux adventifs ou latents peuvent se développer ou sortir.

Ravalement. - Le ravalement est la suppression de toutes les branches jusqu'à leur empatement. Il a pour but d'obtenir de nouvelles branches charpen- 
tières, sur les arbres dégarnis, mal dirigés ou mal faits. Mais pour que l'opération ait du succès, il faut que la tige soit saine. Ciest surtout sur les pyramides et les espaliers qu'il est pratiqué.

Dans les pépinières on le nomme rebottement; il se pratique sur les vieux arbres invendus etsur ceux qui n’ont pas réussi, pour avoir une belle végétation et de beaux rameaux.

Recépage. - Recéper c'est couper net un arbre au collet ou à quelques centimètres au-dessus de la greffe ou de la première bifurcation du tronc ou de la tige. Il ne réussit guère que sur les arbres fruitiers à pépin.

L'entaille ou cran. - Faire une entaille ou un cran, c'est enlever une partie d'écorce et d'aubier sur une branche, pour intercepter le passage de la sève. L'entaille se fait au-dessus ou au-dessous d'un œil ou d'une branche, selon le but qu'on se propose d'atteindre. Quand une branche s'emporte on fait un cran au-dessous et sur l'empatement pour faire dévier la sève. Quand elle reste languissante ou qu'un œil ne se développe pas, on fait un cran au-d.essus pour forcer la sève à s'y porter. Dans ce cas on le pratique à $\mathbf{I}$ ou 8 millimètres de l'œil ou de la branche.

Incision. - Il y a deux sortes d'incision : l'incision longitudinale et l'incision transversale.

L'incision longitudinale se pratique en enfonçant la pointe d'une serpette dans l'écorce jusqu'à l'aubier et la faisant glisser ainsi dans toute la longueur voulue. On l'emploie sur la tige des arbres et sur Jes 
branches dont l'écorce endurcie comprime les canaux de la sève et empêche la circulation.

L'incision transversale se fait en fendant l'écorce tout autour de la branche ou du rameau, pour faire sortir les yeux qui ne se sont pas développés; c'est un diminutif de l'entaille. Elle n'a d'effet que sur des rameaux d'un an ou sur des branches faibles.

Il y a une troisième sorte d'incision, l'incision annulaire. On la pratique en enlevant, soit avec une serpette, soit avec une pince dite à incision, un anneau d'écorce de quelques millimètres de largeur (de 3 à 7 millimètres). Elle a pour but d'entraver la circulation de la sève et par conséquent, de mettre à fruit la partie de la branche qui est derrière elle; tandis qu'elle favorise la production du bois sur la partie qui est en avant. Il ne faut pas en abuser, car elle ruine en peu de temps les arbres les plus vigoureux.

Eborgnage. - Quand un œil est mal placé ou jugé inutile on le fait tomber à l'aide de l'ongle du pouce. On évite ainsi une déperdition de sève, puisqu'il aurait produit un bourgeon qu'on aurait du supprimer plus tard.

L'arqûre est une vieille opération qui est à peu près abandonnée aujourd'hui; elle se fait en courbant en forme de demi-cercle les rameaux ou les branches pour entraver l'ascension de la sève et les mettre à fruit. L'arqûre déforme les arbres et les ruine : on a donc bien fait d'y renoncer.

L'ébourgeonnement. - Ebourgeonner c'est supprimer les bourgeons inutiles. C'est surtout sur la 
vigne, le pêcher et le figuier que l'ébourgeonnement se pratique. Plus il est fait de bonne heure, plus il est avantageux aux arbres qui profitent de la sève que les bourgeons supprimés auraient absorbée en pure perte.

Palissage. - Le palissage joue un grand rôle dans la culture du pêcher; il est très-long et exige des connaissances spéciales; mais pour le poirier et le pommier il est plus simple : on se borne à fixer au treillage les branches charpentières et quelques branches fruitières, suivant les formes qu'on désire donner aux espaliers. Du reste, avec des formes qui sont contre nature, comme celles qu'on voit partout aujourd'hui, le palissage est un travail de toute l'année; tandis qu'en soumettąnt les espaliers à une forme naturelle il est pour ainsi dire inutile, comme on le verra par la suite.

Pincement. - Le pincement est une opération qui a pour but de modérer ou d'arrêter la végétation des bourgeons en faisant passer la sève dans ceux qui ne sont pas pincés, ou d'obtenir des bifurcations o'd bourgeons anticipés nécessaires pour hâter la formation de l'arbre.

On doit pincer le poirier au-dessus de la cinquième ou sixième feuille, aussitôt que les bourgeons en ont huit ou dix. Cette opération se fait pendant que le bourgeon est encore herbacé et qu'on peut facilement le couper avec l'ongle du pouce et l'index.

C'est à l'aide du pincement qu'on met rapidement les arbres à fruit; car dans la branche pincée les yeux se gonflent et se transforment souvent en 
boutons à fruit dès la première année. S’ils se développent et produisent des bourgeons malgré le pincement, on les pince une seconde et même une troisième fois.

Quand un arbre est vigoureux on peut le pincer rigoureusement; mais s'il est faible, il faut le ménager et pincer long. Du reste, comme il faut avoir du bois avant d'avoir du fruit, on a tout le temps de mettre les arbres faibles en rapport. On n'a recours, dans ce cas, au pincement que pour équilibrer la végétation.

On doit ménager les pincements sur les arbres qui sont déjà à fruit; car si on pinçait trop et trop court, la sève refoulée sur les lambourdes et sur les dards les ferait développer à bois et on perdrait ainsi tout le bénéfice de l'opération.

Quand un bourgeon à crochet se présente (une branche bifurquée ou double) on pince la partie la plus rapprochée de la branche charpentière, on laisse végéter la supérieure en entier, et on ne la pince que plus tard, ou l'on n'en supprime que l'extrémité. La sève abandonne alors la partie du bas où les yeux se gonflent et se disposent à tourner à fruit.

Si trois ou quatre bourgeons se présentent sur le même point on n'en conserve que deux, les mieux placés, dont l'un est pincé sévèrement, tandis qu'on laisse le second entier pendant quelque temps; puis on le traite comme nous venons de le dire pour les bourgeons à crochet.

Si l'on a trop attendu pour pincer et que le bour- 
geon soit passé à l'état ligneux, il faut employer le sécateur pour faire les pincements.

Les branches fortes, vigoureuses devront être pincées les premières; tandis qu'au contraire il faut laisser prendre de la force aux bourgeons des branches faibles, parce qu'ils facilitent ainsi leur développement en appelant la sève à eux.

Si le nombre des bourgeons à pincer était considérable sur une branche, il faudrait, pour ne pas produire un refoulement trop intense, procéder à plusieurs reprises.

Enfin, dans les cas où l'on s'aperçoit que les résultats du pincement ne se produisent pas, il est prudent de ne pas trop les multiplier et d'attendre les tailles d'août pour compléter l'opération, en employant les moyens dont nous allons parler.

Taille d'été. - Cette taille est pratiquée sur les arbres fruitiers à noyau et à pépins; mais plus spécialement sur le pêcher; comme nous ne nous occupons pas de cet arbre nous n'avons pas à indiquer la manière de la lui appliquer ; nous nous bornerons donc à donner les détails qui concernent les fruits à pépins.

C'est lorsque la sève se ralentit qu'on fait la taille d'été, c'est-à-dire en août. Elle a pour but principal de compléter la taille d'hiver et les pincements. Ainsi, on supprime, à partir de la troisième ou quatrième feuille, tous les bourgeons qui ont été conservés entiers lors du pincement ou du cassement. Cette opération dispose les yeux de la partie conservée à se mettre à fruit. 
De mème que pour le pincement, quand un arbre nécessite de nombreuses amputations ou suppressions de bourgeons, il faut procéder à plusieurs reprises pour ne pas faire développer trop activement les yeux qui ne doivent pas donner de nouveaux bourgeons, mais simplement se gonfler, s'allonger ou s'arrondir suivant qu'ils se transforment en dards ou en boutons à fruits.

La taille d'été remplace aussi en partie le cassement dont nous allons parler; mais une chose importante à remarquer c'est qu'elle favorise le développement des yeux latents qui se trouvent sur les vieilles branches faibles. Pour cela on taille sur les nœuds, sur les rides où il existe de ces sortes d'yeux qui se développent et se transforment en bouton à fruit. La coupe doit être faite horizontalement, pour ne pas détruire les yeux.

On se sert du sécateur pour faire cette taille, de préférence à la serpette, parce que les branches étant faibles, les plaies sont nulles; qu’il expédie plus vite la besogne et qu'il est plus commode à manier.

Le cassement est une opération qu'on commence à abandonner, parce qu'il est peu nécessaire sur des arbres qui ontété pincés à temps et convenablement, puis la taille d'été le rend, en quelque sorte, inutile. On ne l'emploie que sur les arbres à fruits à pépins.

C'est sur les rameaux faibles et trop longs pour faire des branches à fruits qu'il se pratique. Pour opérer le cassement on place la serpette sous la branche, on appuie le pouce directement au-dessus 
de celle-ci et on la casse en la renversant sur le côté.

Le cassement doit se faire à 6 ou 7 centimètres de longueur, et sans détacher la brindille cassée. Cette opération a pour but de ralentir le cours de la sève dans le rameau cassé, de faire grossir les yeux qui sont à sa base et de les disposer à se mettre à fruit.

Le cassement produit une plaie plus difficile à cicatriser que la coupe ou la taille; il épuise plus le rameau et par cette raison le met plus promptement à fruit.

On fait le cassement aux mois de juillet et d'août et même de septembre. On peut le faire aussi au moment de la taille d'hiver.

DE L A S È VE

Il nous importe peu de savoir ce que c'est que la sève ; ce qui nous importe c'est de connaître comment elle prend naissance, la manière dont elle circule, dont elle se répartit dans toutes les parties d'un arbre et les effets qu'elle produit selon qu'elle est dirigée de telle ou telle manière.

Nous savons que la sève s'introduit dans les plantes par les racines et même par les feuilles qui pompent l'humidité de l'air.

La sève s'introduit dans les racines par les spongioles, monte par le corps ligneux et s'élève jusqu’à 
l'extrémité des rameaux les plus élevés, avec d'autant plus de force que les pores sont plus développés et plus nombreux, c'est-à-dire l'arbre plus jeune.

La sève est mise en mouvement par la chaleur, la lumière et probablement aussi par l'électricité. Elle se porte de préférence dans les branches verticales; elle circule plus lentement dans celles qui sont horizontales et plus difficilement encore dans celles qui sont inclinées vers la terre, au-dessous de la ligne horizontale.

Des expériences ont démontré à Boitard que la sève est chassée en haut par la dilatation de l'air dont ses molécules sont entourées. Ainsi, imaginez un tube rempli d'air ou d'eau, comme un tube plein de mercure qu'on aurait agité. Soit une goutte d'eau, un atome d'air et ainsi de suite. Le calorique dilate l'air qui chasse l'eau; si le calorique diminue le mouvement s'arrête, ce qui explique pourquoi il n'y a plus de sève en hiver et à l'époque où la terre est très-sèche. La sève s'arrêtant dans le premier cas faute de chaleur et dans le second faute d'eau.

Des branches elle passe dans les feuilles où la partie aqueuse éliminée en partie, se transforme en cambium, c'est-à-dire en matière molle, d'abord, qui devient solide ensuite et forme l'aubier et le liber. Ce sont ces couches successives qui constituent l'accroissement de l'arbre et de toutes ses parties.

Les théoriciens ont avancé que la sève avait deux courants : l'un ascendant, l'autre descendant. C'est en vain qu'ils ont essayé de démontrer cette hypothèse qui est aussi fausse qu'inutile. La 
plupart d'entre eux, aujourd'hui, n'émettent plus cette opinion que très-timidement, car ils sont de plus en plus embarrassés pour la justifier.

La sève n'a qu'un courant : elle est ascendante et non descendante.

DE LA CORRÉlation DES RaGiNes AVEG LES BRANGHeS

On fait des questions de tout et à propos de tout. Les choses les plus futiles occupent certains écrivains pendant des années entières, et cela pour résoudre des questions absurdes ou résolues depuis longtemps.

Il y a un demi-siècle, quelques écrivains étrangers aux faits physiologiques, nous posaient en principe que la végétation souterraine était en parfaite corrélation avecla végétation aérienne, c'est-àdire que non-seulement les branches fortes d'un côté de l'arbre annonçaient que fatalement les racines du même côté étaient également fortes; mais qu'elles affectaient les mêmes formes.

On va plus loin aujourd'hui, on dit, et quelques théoriciens affirment qu'il doit en être ainsi, que les racines ont une telle corrélation avec les branches que si elles affectent, par exemple, la forme d'un V sextuplé, les branches devront fatalement tendre à la même forme. Que si elles poussent confusément, il en sera de même des branches. De là, on induit qu'il faut tailler les racines an moment de. 
la plantation comme on taillerait les branches de la tète de l'arbre. C'est le comble du ridicule.

Il y a des gens qui s'évertuent à faire de la science, et qui après avoir rêvé une impossibilité ou un système fantastique, se mettent à raisonner et à déduire des conséquences; leurs innovations ne sont que des vieilleries. En effet, il y a longtemps que ce rêve de la corrélation des branches et des racines a été mis au jour et rejeté. La pratique est là pour démentir cette théorie. Tous les jours on arrache des arbres jeunes et vieux; est-ce qu'on a jamais remarqué rien de semblable? D'ailleurs, il est très-facile, quand on s'expliqúe la circulation de la sève, de comprendre qu'il est de toute impossibilité qu'il y ait la moindre apparence de vérité dans ces suppositions. La sève monte comme dans un tube et s'équilibre ; s'il en était autrement, l'un des côtés grossirait plus que l'autre, s'allongerait davantage et finirait par donner à la tige l'aspect d'un cerceau. Dire que la confusion des racines fait la confusion des branches, c'est renchérir sur cette im. possibilité.

Du reste, tout cela n'est pas nouveau ; car voici ce que nous lisons, à ce sujet, dans la Physiologie végétale de Boitard : “ On a dit qu'il existe une telle » relation entre les racines et les branches d'un " arbre, que si l'on coupe quelques branches, les " racines correspondantes en souffrent, et vice versâ.

»Le fait est que, lorsqu'on altère un organe essen„ tiel d'un végétal, tout le reste de l'individu en - souffre ; mais, quant à cette prétendue correspon- 
" dance de branche à racine et de racine à bran» che, l'expérience m'a prouvé cent fois que c'est

" une véritable chimère. "

Il serait inutile d'entrer dans de plus grands développements physiologiques pour démontrer l'inanité de cette singulière idée; l'opinion de Boitard pour nous fait loi. Les auteurs de ces fantaisies seraient fort embarrassés d'expliquer physiologiquement pourquoi et comment cette corrélation peut exister.

PRINGIPES DE LA TAILLE

Il y a deux siècles que Jean de la Quintinie posa les principes de la taille des arbres fruitiers ; depuis cette époque il n'y a rien eu à y ajouter. Tous les auteurs qui ont traité ce sujet n'ont fait que les formuler en d'autres termes; mais parmi eux il y en a un qui a mis tant de précision dans ces règles qu'on l'a copié bien souvent, nous voulons parler de Boitard !.

La plupart des auteurs modernes lui ont emprunté les quelques, lignes qu'il a écrites à ce sujet, sans jamais le citer ; il est vrai qu'ils ont eu le soin de retourner les phrases et de les dénaturer plus ou moins. Nous croyons qu'il eût été préférable de citer textuellement et de nommer l'auteur.

1. Physiologie végétale de Boitard, 1 vol. avec gravures : 3 francs Encyclopédie de Roret, rue Hautefeuille, 12, Paris. 
Quant à nous, nous empruntons les vingt principes à Boitard et nous le citons. Nous nous bornerons à les faire suivre de quelques observations sur la manière de les appliquer.

$1^{\circ}$. La vigueur d'un arbre dépend, en grande partie, de l'égale répartition de la sève dans toutes les branches.

Pour que la sève se répartisse également dans un arbre, il faut que la charpente soit symétrique; qu'il ait autant de branches d'un côté que de l'autre, qu'elles occupent les mêmes positions respectives ; qu'il y ait autant de feuilles et de fruits sur les unes que sur les autres; quel'espace occupé soit le mệme à droite qu'à gauche. Quand nous disons le méme nombre de feuilles, nous n'entendons pas parler d'une proportion numérique égale ; mais bien d'un ensemble à peu près équivalent.

2०. La vigueur et la durée d'un arbre dépendent, en grande partie, du constant équililibre existant entre ses branches et ses racines.

Plus un arbre a de racines, plus il végète vigoureusement, moins il en a plus il est chétif. Favoriser le développement des racines c'est augmenter le nombre de branches. La suppression des fruits, la taille courte, les engrais, les labours superficiels, le paillage sont autant de moyens d'activer la formation des racines.

$3^{\circ}$. La sève tendant toujours à monter, des racines aux branches le plus verticalement possible, elle abonde dans les branches droites au détriment des autres.

Si une branche prend trop de développement il suffit de l'incliner pour que la sève se ralentisse; 
Si une branche est faible en la redressant verticalement, elle reprend de la vigueur. Si l'on taille court une branche vigoureuse et long un branche faible la sève abandonne la première pour passer dans la seconde. Si l'on supprime les fruits sur la branche faible et qu'on les conserve sur la branche forte, celle-ci s'affaiblit et l'autre se fortifie. En pinçant les bourgeons de la branche forte on l'affaiblit.

$4^{\circ}$. La sève développe des bourgeons beaucoup plus vigoureux sur une branche taillée court que sur une taillée long.

La même quantité de sève étant répartie sur un moins grand nombre d'yeux, les bourgeons sont naturellement plus forts; mais il ne faut pas en induire qu'il faut tailler court les branches faibles et long les branches fortes; ce serait opérer en sens inverse; il s'agit ici de tailler long ou court un arbre tout entier, et non une seule branche. Les boutons à bois sont les écluses par où s'échappe la sève ; or, plus on en laisse plus on ouvre d'issues à la sève et plus les bourgeons sont faibles.

$5^{\circ}$. La sève tendant toujours à affluer à l'extrémité des branches, développe le bourgeon terminal avec plus de vigueur que les latéraux.

L'œil terminal étant plus développé que les autres, il attire plus de sève à lui ; on doit donc le supprimer si l'on ne veut pas, allonger la branche et anéantir les yeux latéraux qui sont à la base.

$6^{\circ}$. Si l'on supprime entièrement une branche, la sève profite aux branches et aux rameaux voisins.

La sève ne trouvant pas d'issue pour s'échapper 
par une branche qu'on à supprimée en entier, ou en très-grande partie, passe dans les voisines, forcément. Supposez un tube bifurqué par où l'eau s'écoule; si vous supprimez l'un des tubes ou que l'ouverture de l'un soit fermée, l'eau passera par l'autre; il en est de même pour la sève.

$7^{\circ}$. Les branches dans lesquelles la sève affue beaucoup, produisent beaucoup de bois et peu de fruits; celles, au contraire, oi elle ne se porte pas avec une. grande abondance produisent beaucoup de fruits et peu de bois.

Ce principe a été developpé dans ce que nous avons dit précédemment; il est donc inutile que nous en démontrions l'application de nouveau ici.

$8^{\circ}$. Plus la sève est entravée dans sa circulation, plus elle produit de rameaux et de boutons à fruits.

Les branches et les rameaux très-vigoureux qui offrent des canaux verticaux à la sève, ou qui s'élèvent perpendiculairement à la tige, ne donnent que du bois et pas de fruits, parce que la sève y étant trop active n'y développe que du bois ; mais les branches inclinées qui forment des ramifications avec les branches verticales ne recevant que peu de sève donnent promptement et facilement des boutons à fruits.

$9^{\circ}$. Toute branche ébourgeonnée ou pincée produit, par la surabondance de la sève qui ne trouve pasà se faire jour en développant du bois, une grande quantité de rameaux et de boutons à fruits.

En effet, si l'on pince un bourgeon à trois ou quatre yeux; ces yeux se développent ordinairement 
et produisent ou des dards ou des brindilles ou des boutons à fruit, comme nous l'avons dit à l'ar ticle pincement.

$10^{\circ}$. Plus on force un arbre à donner du fruit, plus on l'épuise; plus on le maintient en bois, plus on augmente sa vigueur.

Les feuilles, comme nous l'avons vu, concourent à la nourriture de l'arbre; plus il y a de bourgeons plus il y a de feuilles; plus il y a de feuilles plus il y a d'agents'qui concourent à la respiration et à l'alimentation de l'arbre. Les fruits, au lieu de procurer de la nourriture à l'arbre, absorbent la sève à leur profit, donc : plus l'arbre a de fruits, plus il s'épuise.

$1^{\circ}$. Les boutons à fruit, selon les espèces naissent ou sur l'extrémité des rameaux, ou le long des branches.

Chaque espèce d'arbre doit être taillé selon sa nature ou sa variété. Un poirier ne se taille pas comme un pêcher.

12․ Les boutons à fruits, dans les espèces à pépins, naissent le plus ordinairement sur le vieux bois, et dans les fruits à noyaux sur le bois d'une année.

Généralement les boutons à fruits mettent plusieurs années à se produire dans les arbres à pépins, tandis que dans les arbres à noyaux, ils se trouvent en abondance, tous les ans, sur les jeunes rameaux. (Voir ce que nous avons dit à l'article boutons à bois et boutons à fruits.)

13․ Dans les arbres à fruits à pépins tous les gemmes, en se développant, sont organisés de manière à pouvoir produire, selon les circonstances, des boutons a bois, des brindilles ou des lambourdes. 
Pour ne pas nous répéter, nous renvoyons à ce que nous avons dit aux articles pincement, entailles, et cassement, four l'application de ce principe.

14. Dans les arbres à fruits à noyaux, les boutons à fleurs naissent ordinairement sur le bois de l'année, el ne peuvent se métamorphoser en boutons à bois.

Ce principe est tellement explicite, qu'il serait inutile d'y ajouter quelque chose (voir à la taille $d u$ prunier et du cerisier).

1 ${ }^{\circ}$. Tout bouton à fleur, dans les arbres à fruits $\grave{a}$ nogaux, reste stérile s'il n'est accompagné d'un bouton à bois.

Cela est vrai, en général, aussi ne faut-il jamais opérer la taille sur un œil ou bouton de cette nature; cependant, il arrive souvent que ces sortes d'yeux donnent des fruits qui parviennent à maturité ; mais rarement ils sont de bonne qualité. Nous croyons devoir faire cette observation comme renseignement ; et non comme critique.

$16^{\circ}$. Toute branche à fruits, de pécher, lorsqu'elle a donné son fruit n'en donne plus.

Ce principe est parfaitement vrai ; mais il est essentiel de le compléter; à chaque taille on doit ménager un ou deux yeux à bois, pour obtenir une ou deux branches à fruits pour l'année suivante (c'est ce qu'on pourrait nommer la succession des branches à fruits). De cette manière la même branche à fruits ne cesse de produire tous les ans, ou du moins, tous les ans elle est pourvue de boutons d̀ fruits.

17. Les feuilles servent à la respiration des végétaux. Tout arbre qui en est dépouillé en partie, est altéré 
dans sa santé; s'il en est dépouillé en totalité, il risque de périr.

Nous avons précédement à l'article, de quoi se compose un arbre, donné des explications qui corroborent ce principe.

18․ Toute branche ou rameau autour duquel l'air, la lumière et la chaleur ne peuvent circuler, s'allonge, devient maigre, fluet, et ne produit plus ni fruits ni bois.

L'air, la lumière, la chaleur faisant partie de la nutrition des plantes, il est tout naturel qu'elles cessent de vivre ou de produire dès qu'elles en sont privées; car, pas plus que les animaux les végétaux ne peuvent vivre, privés de nourriture et de respiration.

19. Le vieux bois ne produit des bourgeons que lorsqu'il y est forcé par la taille ou par l'altération du jeune bois qui termire la branche.

Ce principe est suffisamment développé à l'article ravalement et rapprochement.

$20^{\circ}$. Tout bourgeon développé hors du temps des deux sèves, reste le plus souvent stérile, maigre et incapable de produire ni bois ni fruits.

La sève du printemps produit plus de bois que de fruit, et celle du mois d'août plus de fruit que de bois. Les bourgeons développés entre ces deux époques, à moins qu'ils ne soient dùs à des pincements, manquent de ces deux caractères.

Boitard termine en disant : "Il est certain que tout cultivateur qui portera la serpette sur un arbre sans avoir ces vingt préceptes présents à la mémoire, 
et sans en faire l'application, ne pourra jamais réussir à obtenir de cette opération les résultats que l'on en attend. ")

MOYENS DE RÉPARTIR ÉGALEMENT LA SĖVE

Si l'on s'est bien pénétré de ce qui a été dit des fonctions des feuilles, des boutons à bois et à fruits, du pincement et des principes ci-dessus, on aura bien vite trouvé le moyen d'équilibrer la sève dans toutes les parties d'un arbre, et nous pourrions, en quelque sorte, nous dispenser d'écrire ce chapitre ; mais au risque de nous répéter, nous allons donner ces moyens aussi succinctement que possible.

Il faut que la charpente d'un arbre soit régulière et symétrique dans toutes ses parties, il faut que toutes les branches soient de même force et qu'elles occupent les mêmes positions respectives, qu'elles portent la même quantité de feuilles et de fruits. Aussitôt qu'on s'aperçoit que la sève se porte plus sur une partie que sur l'autre il faut immédiatement combattre cette tendance et rétablir l'équilibre par tous les moyens que nous avons indiqués et que nous indiquerons encore plus loin.

Il y a dans le défaut d'équilibre de la sève deux effets : une ou plusieurs branches sont trop vigoureuses, ou une ou plusieurs branches sont trop faibles. Voici ce qu'on doit faire dans chaque cas.

Branche trop vigoureuse. - Pour affaiblir une 
branche trop vigoureuse, il faut : tailler court, pincer rigoureusement tous les bourgeons, à plusieurs reprises pour refouler la sève vers les parties faibles; retrancher un grand nombre de feuilles en les détachant de manière à laisser le pétiole (la queue) adhérent au rameau; ébourgeonner s'il y a lieu, faire des entailles à la base de la branche ou du rameau. Laisser, enfin, toutes les brindilles pour déterminer la production du fruit; mais il faut les casser à chaque taille, c'est-à-dire à la taille d'hiver et à celle d'août, et au besoin même pincer les bourgeons qui se trouveraient le long de ces brindilles s'il s'en développait.

Branche faible. - Pour donner de la vigueur à une branche faible, il faut la tailler long ou même pas du tout, si elle porte beaucoup d'yeux à bois, et qu'elle soit saine, afin d'y attirer la sève ; mais si elle avait peu d'yeux à bois et beaucoup d'yeux à fruits, il faudrait la tailler de moyenne longueur sur un œil à bois, afin d'obtenir un bon rameau. On supprime les fruits, on laisse tous les bourgeons se développer, sans les pincer ni les casser, on incise les écorces durcies, on fait des entailles au-dessus de la branche pour forcer la sève à y passer.

En conséquence si un arbre présente à la fois des branches fortes et des branches faibles il faut traiter les unes et les autres comme nous venons de le dire.

N'admettant pas de formes d'arbres à branches inclinées, palmettes, espaliers carrés, etc., nous ne parlons ni de l'écartement des branches pour les 
éloigner des murs, ni de leur redressement, ni de leur inclinaison.

Le palissage pour les fruits à pépin étant chose à peu près insignifiante nous nous sommes tenu, à son égard, dans les limites les plus simples; car les moyens indiqués ci-dessus seront toujours suffisants pour équilibrer la sève dans un arbre et la bien répartir.

T R E I L I A G E

Le treillage le plus économique et le plus simple est celui que nous allons indiquer.

On tend horizontalement le long d'un mur, des tils de fer galvanisé, n ${ }^{0} 17$, à la distance de 25 centimètres. Le premier est à 20 centimètres du sol ; les supporls sont tous les 7 mètres au plus et on met un raidisseur pour 30 mètres de longueur de fil de fer.

Les supports sont de petites pattes trouées qu'on scelle dans le mur et dans lesquelles en fait passer le fil de fer.

Cela fait, on place, tous les 25 centimètris et verticalement, des baguettes en bois sulfaté d'un centimètre et demi carré, peint si l'on veut, de manière à former des mailles de 25 centimètres.

Pour le pêcher dont les petites branches doivent ètre moins espacées il ne faudra écarter les baguettes que de 1 כ̆ centimètres.

Nos treillages sont fait de cette manière, et nous 
en avons la plus grande satisfaction : ce système réunit tous les avantages : solidité, propreté, économie et durée.

Le cerisier, le prunier sont également très-bien sur un treillage de $2 \breve{3}$ centimètres carrés.

\section{DE LA FORME DES ARBRES}

La forme à laquelle on soumet les arbres est trèsimportante ; elle varie suivant les besoins, l'espèce, la nature du sol et le climat. Les plus usitées sont:

$1^{\circ}$ Le plein vent ou haute tige; $2^{\circ}$ la touffe ou vase; $3^{\circ}$ la pyramide ou cône ; $4^{\circ}$ l'espalier; $5^{\circ}$ le cordon.

Le plein vent ou haute tige s'emploie pour la formation des vergers, toutes les essences d'arbres peuvent s'accommoder de cette forme.

La touffe ou vase convient également à tous les arbres, on n'emploie cette forme que dans les jardins de produit où l'on ne tient pas au coup d'œil. Elle n'exige, du reste, que fort peu de connaissances et de main-d'œurre ; le premier venu peut être dressé en quelques jours à la tenue de ces arbres.

La pyramide ou cône. Presque toutes les essences peuvent être élevées en pyramides; toutefois le pornmier y est très rebelle et ne fait jamais de beaux arbres. Cette forme est presque exclusivement réservée au poirier; il y a même des variétés à branches divariquées qui sont difficiles à dresser, d'autres se ramifient mal: il y a un choix à faire. La pyramide 
peut se dresser dans tous les sols; mais elle exige des connaissances sérieuses pour être convenablement établie et entretenue.

L'espalier convient à tous les arbres ; mais généralement cette forme n'est appliquée qu'aux variétés dont le produit ne serait pas assuré en plein air, il se dresse comme l'on sait le long d'un mur. On donne le nom de contre-espalier à ceux qui sont établis sur des treillages éloignés des murs.

Pour bien dresser, bien gouverner un espalier il faut posséder à fond les principes de la taille, surtout si on adopte certaines formes de fantaisie. Nous indiquerons plus loin celle qui est la plus convenable et qui exige le moins de soin et de frais.

Le cordon. - On donne le nom de cordon à l'arbre qu'on fait courir horizontalement ou à peu près, le long d'un fil de fer. On nomme à tort, cordonvertical, cordon oblique, les arbres qui sont dressés les uns perpendiculairement, les autres obliquement : cette dénomination ne signifie rien. Il serait plus rationnel de les appeler tiges verticales, tiges obliques.

Le poirier et le pommier réussissent très-bien en cordons, et produisent de très-beaux fruits.

On peut établir des cordons dans tous les sols, avec succès. Cette forme a l'avantage de procurer des fruits rapidement et de permettre de grouper un grand nombre de variétés sur un espace très-restreint. La taille en est facile.

L'inconvénient du cordon est de ne pas vivre longtemps ; mais il a l'avantage de se remplacer aisément. 
TAILLE DU POIRIER A HAUTE-TIGE

On nomme haute tige ou plein vent, un arbre qui a 1 mètre 5̆() à 2 mètres 2.3 de tige sans branches.

Ces arbres ne sont soumis à la taille que pendant les deux ou trois premières années de leur plantation; passé cette époque, on ne leur fait que quelques émondages, comme nous le dirons plus loin.

Voici les soins que réclame un arbre à haute tige après qu'il est planté, comme nous l'avons indiqué. On ne supprime aucune branche lors de la plantation, seulement quand la végétation s'est mise en mouvement, que les feuilles sont bien développées, que les jeunes bourgeons commencent à paraître, ce qui a lieu généralement à la fin du mois de mai, on procède à la taille pour former la charpente ou la tête de l'arbre. Au printemps suivant on opère comme il suit.

Première année, première taille. - S'il n'a poussé qu'une tige pyramidale et des branches latérales mal placées ou difformes, on l'étête au-dessus de la troisième branche; on raccourcit celle-ci à trois yeux ou sur le second ou troisième bouton, en choisissant celui qui est placé en dessous de la branche. Là se borne la taille de la première année. On laisse croître tous les bourgeons en liberté. Cependant dans les variétés à branches divariquées telles que le bigarreau noir dans les cerisiers et le beurré diel 
dans les poiriers, il est bon de relever les bourgeons et de les palisser à quelques baguettes fixées autouı de la tige.

Si l'arbre a dcux, trois ou quatre branches, on laisse développer les feuilles, on taille ces branches sur deux bons rameaux et à défaut sur deux yeux convenablement placés pour établir la charpente.

La charpente d'un arbre à plein vent doit se composer de deux, trois ou quatre branches-mères, disposées de manière à lui donner la forme d'un entonnoir.

Ainsi, avec deux branches, l'arbre représente un $\mathrm{V}$; on fait bifurquer chacune d'elles de manière à en avoir quatre, ce qui donne, alors, ia forme d'un autre $\mathbf{V}$ sur chaque branche et ainsi de suite.

Deuxième année, deuxième taille. - On continue de tailler assez court cette année pour former la tête de l'arbre, comme nous venons de l'indiquer. Alors il aura quatre rameaux à l'automne, tous les autres bourgeons seront rigoureusement pincés, au-dessus de la cinquième feuille.

Troisième année, troisième taille. - Taillez tous les rameaux de la charpente à 40 centimètres de long et toutes les brindilles au tiers de leur longueur. $\boldsymbol{A}$ cet âge l'arbre doit ètre à peu près formé; il aura huit rameaux, ou huit branches charpentières bien établies, même en admettant qu'il n'en avait que deux à la première année, puisque ces rameaux au- . ront été bifurqués une fois à la première taille, ce qui en donne quatre, et une seconde fois à la deuxième ce qui en produit huit. 
A partir du moment où vous avez obtenu huit mères-branches, il ne s'agit plus que de les allonger, de les diriger symétriquement pour qu'elles ne soient pas plus inclinées les unes que les autres.

S'il y en a une qui prend trop de développement, raccourcissez-la un peu, supprimez ou raccourcissez ses branches latérales. Si une autre s'incline relevezla; si elle est trop verticale ou droite, écartez-la à l'aide d'un arc-boutant en bois. Si l'arbre est un peu divariqué, qu'il tienne mal ses branches, placez un cerceau au centre de la tête et fixez-les y suivant les besoins. Alors il sera régulier, symétrique; il n'y aura plus qu'à en évider le centre pour lui donicer la forme d'un gobelet, d'un $\mathrm{V}$ ou d'un U, selon la manière dont les branches charpentières auront pris naissance. Toutes les branches qui tendront à baisser, à se rapprocher du sol, et même à venir à l'horizontale devront être supprimées ou relevées pour conserver à l'arbre sa vigueur, sa croissance, et la beauté du coup d'œil.

Nous venons de dire que l'arbre traité avait huit branches charpentières; mais il arrive fréquemment qu'au lieu de deux branches on peut en avoir trois; en les taillant sur deux bons yeux, à la seconde année, on se trouve en avoir six. Ce nombre est suffisant pour faire une belle tête; car la nature les développe en raison de l'espace libre et on obtient le même résultat, les mêmes produits qu'avec huit. 
TAILLE DES ARBRES EN TOUFFE, EN GOBELET, FN YASE, EN GÉPÉE

Nous ne ferons aucune distinction entre les arbres dits en touffe, en vase, en gobelet, en cépée; parce qu'à part la dimension, le mode de taille est le même ou devrait l'être.

Ces arbres s'établissent et se gouvernent comme les plein vent; et n'en diffèrent que parce qu'ils n'ont pas de tiges et qu'on les fait se bifurquer et se ramifier à 30 centimètres du sol.

Onplante des scions d'un an de greffe et on les étête au mois de mai, à $1 \%$, 20 ou 2.5 centimètres du sol, pour obtenir deux, trois, quatre ou même cinq branches charpentières. Chaque branchese bifurque tous les ans à 60 ou 70 centimètres de longueur, de manière à former un vase, un gobelet, à l'aide d'une série de $\mathrm{V}$ placés les uns sur les autres.

Pour que ces sortes d'arbres soient productifs, de longue durée et gracieux, il faut que les branches charpentières partent du tronc pour s'élever à l'angle

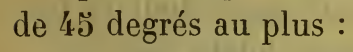

Chaque branche doit être garnie de productions fruitières, comme dans les pyramides également sans bifurcations.

Il y a d'autant plus de branches charpentières que l'arbre est plus haut; elles doivent ètre espacées entre elles de อ̃0 centimètres au plus. Elles prennent naissance comme celles des plein vent sur deux, trois ou quatre branches principales pour se diviser 
en se bifurquant autant que cela est nécessaire et régulièrement à la même hauteur.

On peut aussi laisser une tige de 50 à 60 centimètres de hauteur et prendre huit ou dix branches tout autour comme on le ferait d'une pyramide tronquée. Alors on ne bifurque pas les branchesmères.

TAILLE DE LA PYRAMIDE OU GÔNE

Pour obtenir de belles pyramides, d'une longue durée, d'un bon rapport, il faut planter des scions d'un an de greffe; on les raccourcit à 50 centimètres de hauteur, aussitôt qu'on s'est ąssuré de la reprise sur un bon œil à bois, et de manière à faire développer tous les yeux à partir de 25 à 30 centimètres du sol. On fait cette première année lemoins de pincements possible.

Pour se faire une idée exacte de la manière dont on doit tailler, il faut se rappeler qu'une pyramide doit avoir la forme d'un cône allongé, dont la base ait environ un tiers de la hauteur totale (fig. 44.)

Deuxième année de plantation, deuxième taille. La sève se portant toujours de préférence au sommet de l'arbre, les rameaux produits par la première taille sont généralement plus longs dans le haut que dans le bas; cependant il arrive parfois que c'est le contraire qui alieu, d'autres fois, l'arbre n'a presque rien poussé, la végétation a été pour ainsi dire nulle 
et les yeux ne se sont pas développés. A la seconde. taille, l'arbre peut donc présenter trois états différents : $1^{\circ}$ Les rameaux du haut forts, ceux du milieu faibles, ceux $d u$ bas très-faibles. $2^{\circ}$ Les rameaux $d u$ haut et du bas à peu près équilibrés, c'est-à-dire, de la même force. $3^{\circ}$ Quelques rameaux dans le haut etles yeux du bas non développés.

Voici comment on doit opérer dans les trois cas :

Le numéro 1 sera taillé comme il suit :

Les branches inférieures, très-faibles, seront laissées dans toute leur longueur ; celles du milieu, suivant leur force, seront raccourcies du côté du bas à cinq ou six yeux, les suivantes à trois ou quatre yeux ; celles de l'extrémité supérieure à deux yeux et à un œil ; puis on taille la flèche à deux, trois ou quatre yeux, suivant la force du sujet et toujours sur un œil opposé à celui sur lequel la taille précédente a été faite, afin de la redresser. $\mathrm{Si}$, dans le milieu ou dans le haut, il y avait une branche faible on la taillerait un peu plus long. Notez que toutes les branches charpentières doivent être taillées sur un œil placé en dessous de la branche ou de côté, mais jamais en dessus.

Le numéro 2 présentant un équilibre presque parfait on taillera de manière à le conserver, savoir : les branches inférieures à cinq ou six yeux, les suivantes à quatre ou cinq, et ainsi de suite en diminuant graduellement jusqu'à un œil. La flèche sera raccourcie sur un œil placé en sens inverse de la greffe, à trois, quatre ou cinq yeux, selon la force de l'arbre. Le numéro 3 offre un arbre dont la végétation est 
très-mauvaise. Il faut tailler tous les rameaux du haut à un œil ou sur l'empâtement ou couronne, puis faire un cran derrière chacun des yeux qui ne sont pas développés.

Pendant toute l'année on surveillera la végétation pour la régulariser. On pincera les bourgeons qui prendraient trop de force, pour refouler la sève sur les plus faibles. Si les branches charpentières, auxquelles on a conservé quatre, cinq ou six yeux, se bifurquaient, il faudrait pincer ces bifurcations, pour renvoyer la sève à l'extrémité du rameau de prolongement de la branche charpentière.

Troisième année, troisième taille. - Au momentde la taille, l'arbre doit présenter la forme conique de la fig. 44. On remarque deux étages bien distincts. Le premier, à partir du sol, est composé de branches charpentières avec les rameaux de prolongement; sur le vieux bois il y a déjà des productions fruitières, c'est-à-dire, des dards, ou de petites brindilles. L'étage supérieur est formé de cinq, six ou sept rameaux de l'année y compris la flèche ou le rameau de prolongement de la tige.

La taille d'un tel arbre devient facile, on taille toutes les brindilles du premier étage à deux bons yeux, on raccourcit les dards qui ont plus de 15 centimètres sur 4 yeux et on taille le rameau de prolongement des branches charpentières, suivant sa force et sa position, à six, sept ou huit yeux. Quant au second étage, il se taille comme celui du numéro 1 , si les rameaux supérieurs sont plus forts que les inférieurs ; comme le numéro 2 s'ils sont équilibrés; 
comme le numéro 3 s'il y a des yeux non développés.

On surveillera attentivement la marche de la sève pour opérer le pincement des bourgons vigoureux, et cela toute l'année. - On pincera surtout les bourgeons de bifurcation qui naissent sur les jeunes rameaux et qui nuisent considérablement au prolongement des branches charpentières.

Au moment de la taille on fera prendre à toutes les branches charpentières, ainsi qu'aux rameaux, la position qu'elles doivent conserver toujours, c'està-dire qu'on les rapprochera ou on les éloignera de la tige pour qu'elles forment avec elle un angle de 45 degrés. Pour cela, on place des arcs-boutants en bois, taillés en biseau et creusés à chaque bout pour qu'ils embrassent la branche et la tige, afin de donner l'écartement voulu. Pour opérer le rapprochement on se sert d'osier qu'on ne serre pas trop, dans la crainte de faire naître des bourrelets. On relève ainsi les branches ou les ramẻaux inclinés, déviés, ou trop écartés.

Si la tige se tient mal, si l'arbre a des rameaux tordus ou divariqués, on fixe un tuteur à la tige et on y accole la flèche ou le bourgeon de prolongement.

Si la tige est brisée ou rompue, on choisit un bourgeon bien placé et on le palisse au tuteur pour opérer le remplacement du rameau hors de service.

On s'assure que les opérations. de la taille de l'année précédente ont atteint leur but; au besoin on renouvelle les crans, etc., etc. 
Quatrième année, quatrième taille. — La quatrième taille présente les mêmes caractères que la troisième. Il en sera désormais ainsi pendant toute la durée de l'arbre. A la quatrième année, on remarque que les productions fruitières sont en grand nombre déjà sur le vieux bois; tout annonce que le planteur recevra bientôt la récompense de ses peines : l'arbre va entrer dans la période de production.

La taille est exactement la même que celle de la troisième année ; seulement il faut tenir compte des différences de végétation. Certains arbres ont poussé des rameaux d'un mètre; d'autres n'en ont donné que de 30,40 ou 50 centimètres. Il faut tailler en conséquence.

Remarque. - Il faut dans une pyramide faire tomber, à la taille d'hiver, les deux tiers du bois qu'elle a produit. En d'autres termes, il faut raccourcir les rameaux des deux tiers environ; quant aux brindilles, il ne doit pas y en avoir si l'arbre a été surveillé et pincé à temps. S'il y en a, il faut les taillèr à trois bons yeux, au plus, à deux yeux au moins, sauf à recourir au pincement au besoin.

Cela entendu, la taille devient facile, il ne s'agit plus que d'entretenir l'harmonie dans toutes les branches, en suivant les principes que nous avons donnés.

On continuera tous les ans à allonger les branches de charpente, et à en créer de nouvelles, de manière qu'il y en ait huit ou dix par mètre de hauteur de tige.

Il ne doit y avoir aucune bifurcation sur les bran- 


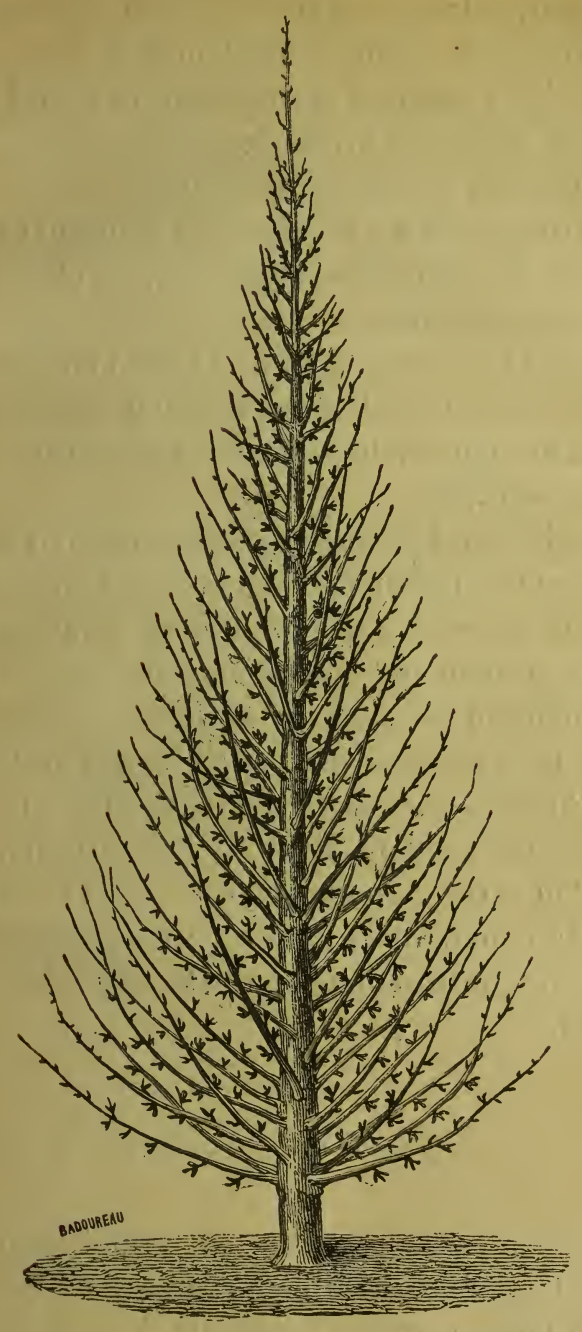

Fig. 44. 
ches charpentières; mais seulement des productions fruitières : dards et lambourdes.

On taillera toujours le côté exposé au nord un peu plus long que celui exposé au midi ; car il se développe toujours moins que ce dernier.

On évitera que les branches se croisent; qu'elles ne soient pas également distancées, qu'elles fassent confusion entre elles.

Une fois l'arbre mis à fruit, il faudra tailler un peu plus court que nous l'avons dit, pour activer la production du bois et diminuer celle des fruits, afin de ne pas le fatiguer.

Les arbres qui paraîtront épuisés devront être raccourcis plutôt qu'allongés; aussi, au lieu de tailler long, on procédera peu à peu au rapprochement puis au ravalement, pour concentrer la sève dans les branches à bois, assurer et augmenter les fruits.

Nous ne pousserons pas plus loin les explications sur le dressage du poirier en pyramide; ce serait nous répéter à chaque taille. Il faut s'attacher à produire un arbre dressé comme on le voit à la fig. 44, en mettant en pratique les principes de la taille et les instructions générales que nous avons données.

F'ORMES DE L'ESPALIER

L'espalier est cultivê sous un grand nơnbre dè formes. Chaque arboriculteur a voulu avoir la sienne. Il y a la forme carrée, la palmette, le candélabre, la 
lyre, le $\mathbf{V}$ ouvert, l'U, le double $\mathbf{U}$, la palmette Verrier, etc., etc.

Toutes ces formes demandent beaucoup de travail, de savoir, de patience et ne font pas des arbres de longue durée, parce que la plupart sont dressés contrairement aux lois de la nature, c'est-à-dire aux principes de physiologie.

Le talent, le grand secret de l'arboriculteur c'est de se rapprocher le plus possible de la végétation normale dans la forme de ses espaliers; or, il suffit de jeter les yeux sur toutes les formes adoptées et prônées aujourd'hui pour voir qu'on s'en est singulièrement écarté. Est-ce que la nature produit des branches horizontales, inclinées? Ce n'est que quand les arbres ont produit plusieurs années durant et qu’ils sont épuisés, qu'ils arrivent à cet état. Or, comment se fait-il que dans toutes les formes nouvelles on ne rencontre que branches inclinées ou horizontales? Il est évident qu'on a voulu faire de la fantaisie et étonner par l'originalité, rien de plus.

Il est incontestable qu'en toutes choses il faut imiter la nature et suivre ses lois, autant que possible. Nous disons autant que possible, car la nature n'ayant pas toujours le même but que celui que nous voulons atteindre, il faut favoriser ses tendances quand elles ne contrarient pas les nôtres. La nature fait des arbres forts, vigoureux et qui ne donnent des fruits que dans leur vieillesse ou, tout au moins, tardivement, afin de se reproduire. Ce n'est pas précisément le nôtre, nous voulons des fruits le plus tòt possible. Il y a un moyen de tout concilier. 
Les arbres poussent des bourgeons qui s'élèvent presque perpendiculairement; s'ils s'éloignent de la verticale ou de la perpendiculaire, c'est pour obéir à une autre loi physiologique, pour trouver de la lumière et de l'air. L'angle décrit par cette déviation est très-faible : il est presque insensible.

Puisque la nature produit, presque toujours, des rameaux verticaux, pourquoi vouloir des formes où les branches sont inclinées à 45 degrés, à 75 degrés et même à 90 degrés ; c'est-dire horizontales ? Fantaisie ! Tour de force qui coûte cher à celui qui l'exécute. Dans les formes contre nature, tous les ans il faut faire tomber, soit à la taille, soit par les pincements des quantités considérables de bois. On croirait que ces arbres sontplutôt destinés à produire des fago ts que des fruits.

Cela entendu et compris, les meilleures formes sont celles dontles branches partent verticalement du tronc avec le moins de déviation possible, nécessitée pour obtenir l'écartement voulu. C'est cellelà que nous avons adoptée, comme on péut le voir à la figure 4.50 .

Il reste à résoudre une question qui est celleci : les grandes formes sont-elles préférables aux moyennes ou aux petites? C'est ce que nous allons examiner.

Quels sont les avantages des grandes formes? Nous n'en voyons pas, si cen'est d'économiser les frais de plantation, puisqu'au lieu de quatre ou cinq arbres il n'en faut qu'un.

Quels sont les inconvénients? Il faut attendre quatre, cinq, six fois plus de temps pour jouir et 
pour garnir les murs ; il faut des terres plus riches, plus de soins. Si un arbre meurt, il laisse un vide considérable qu'il est impossible de combler symétriquement, puisque les arbres qu'on pourra y planter seront en retard sur ceux remplacés; enfin, la récolte est infiniment moindre.

Voilà les raisonsqui nous font préférer les petites formes aux grandes, c'est-à-dire les arbres qui mesurent 1 mètre 50 à 2 mètres de développement à ceux qui en ont 8,10 et plus. Tout au plus admettrions-nous les formes de 4 mètres dans les sols qui ne sont pas d'une grande richesse, et très-profonds.

Les formes qui peuvent le mieux satisfaire aux exigences de la nature et aux besoins de l'homme, étant celles que nous venons d'indiquer, nous avons adopté et nous recommandons la palmette-Journet (figure 45) du nom de l'arboriculteur qui l'a le premier recommandée.

TAILLE DE LA PALMETTE VERTICALE JOURNET

Pour établir cette palmette, comme tous les autres espaliers, il faut planter des arbres d'un an de greffe seulement, afin d'avoir de bons yeux à bois, à la base, pour obtenir facilement les branches latérales.

M. Journet procède autrement qu'on le fait génélement. Voici sa manière telle qu il l'a décrite luimême.

"Lorsque mon sujet est greffé: je pince la flèche 
" quand elle a 30 centimètres de hauteur, environ,

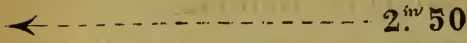

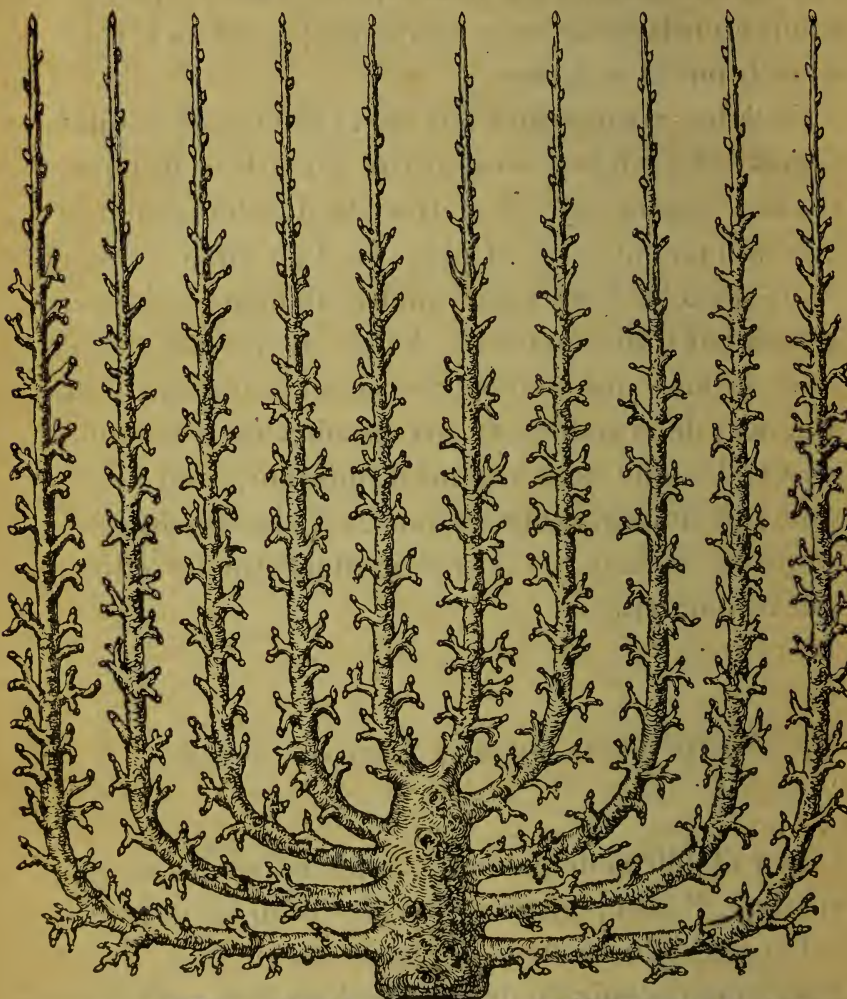

- DAOOREAV

Fig. 45.

" afin de bien développer les neils de la base ; j'égalise 
- la végétation par le pincement des œils les plus

" forts, de manière à développer également leurs " canaux séveux et avoir un égal empâtement sur

„ la tige. Chaque œil doit vivre de sa vie propre,

") sans bifurcation et tout à fait indépendant, comme

v un tube destiné à absorber la même quantité de

» liquide à un réservoir commun. Il faut autant que

v possible, que les œils soient tous placés à la même

„ hauteur, pour être dans les mêmes conditions d'ab-

\ sorption.

"Je conduis chaque branche à la place qu'elle „ doit occuper, en pinçant les branches qui ont le moins de longueur à parcourir, et dès ce moment, ") je les relève perpendiculairement, en conservant à , toutes les branches, la même force, la même hau" teur, le même nombre d'œils, en les faisant tous " également se développer, de sorte que, dès la même ¿ année, mon arbre est formé. "

L'arbre dont parle M. Journet, porte dix branches (voir la figure 45). - Pour obtenir dix branches dès la première année, il faut deux choses : $1^{\circ}$ que l'arbre soit mis en place depuis au moins un an; $2^{\circ}$ que la terre soit d'une richesse exceptionnelle. Comme on est rarement dans ces conditions nous conseillons de procéder autrement.

A la seconde année de plantation on étête l'arbre à 30 centimètres, pour obtenir trois ou cinq branches, selon la force de végétation; on en dirige une ou deux de chaque côté en les inclinant à l'angle de 45 degrés, et on palisse verticalement celles qui sont destinées au prolongement de la tige. 
L'année suivante on taille le rameau de prolongement de manière à faire développer un nombre d'yeux suffisant pour compléter la charpente de l'arbre. On laisse entières les branches formant le premier et le second étage et on les prolonge le plus possible pour arriver à les mettre en place à la fin de l'année. On forme ainsi l'arbre en deux ans, au lieu d'un.

On a soin de pincer les bourgeons qui prennent trop de vigueur, pour équilibrer la sève.

A la troisième année les soins diminuent, on dresse les branches verticalement pour que l'arbre soit exactement comme le représente la fig. 4ว. On pince les bourgeons latéraux, pour empêcher les bifurcations; on entretient l'équilibre entre toutes les branches de charpente. On les allonge chaque année de la longueur que la végétation le comporte, on applique, enfin, les principes de la taille, afin de conserver l'arbre en pleine production et en pleine vigueur aussi longtemps que poss.ble. Si le sol est bon, si l'arbre est très-vigoureux on ne taille pas les flèches si ce n'est pour les équilibrer entre elles.

Remarquez que les étages des branches charpentières ne sont distants que de 10 à 12 centimètres à leur naissance et qu'ainsi les branches fruitières sont presque nulles sur cette partie horizontale. Elles sont du reste inutiles; car le but principal est d'avoir des branches verticales de la plus grande longueur.

La palmette fig. 450 occupe un espace de 2 mètres 50 centimètres, les branches verticales étant à 25 cen- 
cimètres de distance l'une de l'autre; mais on pourrait facilement la restreindre à 2 mètres de développement en n'espaçant les branches charpentières que de 20 centimètres. La hauteur est de 2 mètres 50 centimètres environ, elle pourrait être de 3 et même de 4 mètres.

On peut faire, dans les sols peu favorables au poirier, des palmettes de 1 mètre 50 centimètres, n'ayant que six ou sept branches. A 1 mètre seulement, elles auraient encore quatre ou cinq branches, selon qu'on les espacerait de 25 ou de 20 centimètres.

Cette forme est la plus simple, la plus productive, la plus facile à faire et à gouverner. Elle est la moins onéreuse, car elle n'exige que fort peu de soins; elle est très-durable parce qu'elle se rapproche le plus de la forme naturelle et que l'on n'est pas obligé de combattre toute l'année les productions de la sève comme cela arrive dans les formes de fantaisie où tout est en opposition avec les principes de la physiologie végétale. Elle se prête à toutes les dimensions pratiques, puisqu'on peut faire les espaliers de $1,2,3$ et 4 mètres d'étendue sur telle hauteur. qu'on le désire.

Nous n'insisterons pas davantage sur le mérite de cette forme que nous croyons appelée à remplacer toutes les autres dans les jardins où l'on s'occupe du rapport et de l'économie, plutôt que de la fantaisie ou des tours de force. Du reste, elle est plus gracieuse que les autres, surtout quand on la pratique sur une certaine étendue de murs; car elle présente moins de lacunes et plus de régularité. 


\section{CORDON HORIZONTAL}

TAILLE DU POIRIER

Le poiriers'accommode parfaitement de la culture en cordon horizontal; seulement dans les sols substantiels où il prend un grand développement il faut qu'il soit greffé sur coignassier, sans quoi le résultat serait mauvais. Ces arbres prendraient trop de développement et ils ne fructifieraient pas malgré les pincements réitérés. Il y a cependant plusieurs variétés qui peuvent être mises en cordons, bien que greffées sur franc; telles sont le beurré clairgeau, bergamotte fortunée, bonne de Malines, etc.

Pour établir un cordon, il faut planter des arbres d'un an de greffe, et les palisser de suite le long d.u fil de fer, en dirigeant, autant que possible, l'inclinaison du nord au midi ou du nord à l'ouest, ou de l'est au midi.

On ne les taille pas; on les espace de 2 à 4 mètres, selon la richesse du terrain; on pince tous les bourgeons pour forcer la sève à se porter sur celui de prolongement; on ne souffre aucune bifurcation; on supprime toutes les pousses qui naissent le long de la tige. On ne taille jamais le rameau de prolongement, à moins qu'il ait plus de 50 centimètres. Avec ces simples opérations on met facilement à fruit un cordon de poirier en trois ans. Il ne s'agit plus, alors, que de l'entretenir convenableinent, pour arriver à lui donner la forme de la fig. 46 . 
Si un cordon poussait très-vigoureusement, on pourrait en faire de deux et même de trois étages;

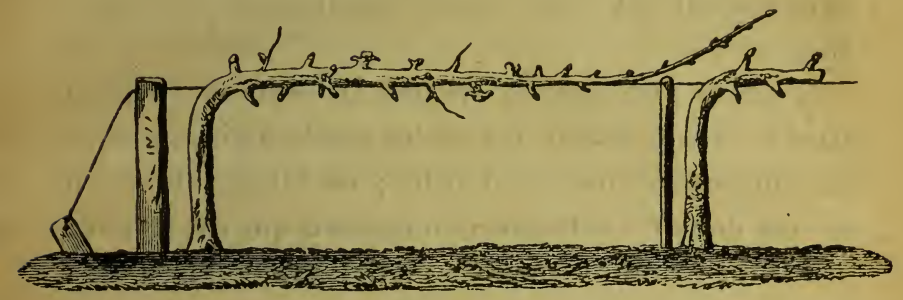

Fig. 46.

pour cela, il suffirait de disposer un second rang de fil de fer parallèlement au premier.

TAILLE DU POMMIER

Le pommier est un arbre de plein vent par excellence; car il réussit mal en pyramide et il est peu agréable en espalier. On peut en faire des touffes ou cépées ; mais elles sont également disgracieuses et peu productives. La meilleure des petites formes pour lui c'est le cordon horizontal. Les fruits qu'il donne sont très-beaux et d'excellente qualité.

Le pommier plein vent se traite absolument comme le poirier. Les cordons de pommier se font aussi comme ceux de poirier; seulement, il est utile, quand on plante des scions qui ont plus de 50 centimètres de couche sur le fil de fer, de les tailler à cette longueur pour que tous les yeux se développent. 
Les pommiers pour cordon se plantent de 1 mètre 50 à 2 mètres de distance selon la qualité du sol, le rameau de prolongement ne se taille jamais, à moins qu'il ait plus de 50 centimètres de longueur.

Il arrive quelquefois, quand la flèche du cordon atteint l'arbre voisin, qu'on les soude en les greffant par approche l'un sur l'autre; on laisse, alors, un œil ou deux à l'extrémité du rameau qui est fix ésur le dos du pommier rencontré. Cet œil se développe, sert d'appelle-sève et se traite ensuite comme une production fruitière ordinaire.

TAILLE DU PRUNIER

Le prunier fait des arbres de plein vent de toute beauté ; il réussiten espalier, mais il fait de mauvaises pyramides et des cordons plus mauvais encore.

Comme arbre à haute tige il se dresse comme le poirier. Seulement, certaines variétés ayant le défaut de produire un grand nombre de branches latérales et fruitières, il exige de fréquents nettoyages pour le débarrasser du vieux bois devenu inutile et qui sert de refuge à des milliers d'insectes qui trouvent, dans les interstices des écorces et dans les vieilles mousses les moyens de se multiplier au détriment de l'arbre.

L'espalier de prunier ne se taille pas comme celui des arbres à pépìns. La branche qui a donné du 
fruit meurt et n'en donne plus. Il faut donc, pour empêcher les vides, raccourcir toutes les branches à fruit à deux yeux pour faire développer deux nouveaux bourgeons. On pince l'un à trois feuilles pour avoir du fruit, l'autre à six pour avoir du bois. De cette manière la branche à fruit se trouve remplacée et vit indéfiniment.

Le prunier réussit parfaitement en PalmetteJournet.

T A IL LE DU GERISIER

Le cerisier est propre au plein vent. c'est même la seule forme sous laquelle il est cultivé en grand. Il fait de belles pyramides, certaines variétés du moins. On en obtient aussi de très-belles palmettes. On peut en faire également des cordons horizontaux; mais sous cette forme il n'y a que les variétés peu vigoureuses qui réussissent.

Le cerisier à plein vent demande suivant l'espèce à ètre tenu en forme pyramidale ou en vase comme le poirier.

Dressé en pyramide ou en espalier, la branche à fruit veut être pincée pour se renouveler. Il faut donc la tenir courte pour en faire le remplacement. On fait cette opération, aussitôt que la brindille a 12 ou 13 centimètres; alors on pince sur la quatrième ou la cinquième feuille, non compris les folioles qui sont à la base. Quand les bourgeons se sont 
développés au-dessous de la partie pincée on pince de nouveau.

A la taille d'hiver, on coupe la brindille à deux yeux, pour avoir deux bourgeons qu'on pince pendant l'été, l'un à̀ trois feuilles pour fructifier, l'autre à cinq ou six feuilles pour obtenir la branche de remplacement. Chaque année on renouvelle ces opérations.

Le cerisier s'accommode très-bien de la PalmetteJournet. 


\section{ACCESSOIRES}

DE LA G ULTURE FRUITIÉRE

Pendant les grandes chaleurs de l'été, les arbres plantés à l'automne ou pendant l'hiver précédent, et surtout au printemps, souffrent de la sécheresse. Il est de la plus grande utilité de les arroser. Pour cela on fait un godet de 10 centimètres environ de profondeur au pied de chaque arbre, on le remplit de litière ou de fumier peu pourri et on arrose une fois par semaine au moins, sur ce paillis, en mettant 15 litres d'eau par arbre. Cette opération doit se faire le soir après le coucher du soleil et, en même temps, on bassine les feuilles.

\section{B A S S IN A GES}

Les bassinages sont des arrosements légers qu'on fait sur les feuilles et sur toutes les parties d'un arbre quand les chaleurs sont fortes et la sécheresse continue. Dans ce cas, l'évaporation par les feuilles est considérable et il y a lieu de craindre le flambage, c'est-à-dire un arrêt de circulation dans la sève qui 
cause la brûlure des feuilles et leur chute ; alors, un bon bassinage fait le plus grand bien. Pour opérer, on se sert d'une pompe à main qu'on place dans un seau plein d'eau qu'on lance tous les deux jours sur les feuilles des espaliers. On néglige cette opération pour les arbres à plein vent, cependant elle serait atile, parfois.

\section{DU PAILLIS}

Dans les sols secs un bon paillis est toujours indispensable. Il active la végétation des jeunes plantations et assure le fruit de celles qui sont en rapport.

Pour pailler, on met au pied de chaque arbre, 10 centimètres, environ, de litière ou de fumier peu pourri, sur un rayon de $ّ 0$ centimètres pour les jeunes arbres, et d'un mètre carré pour les arbres en rapport. Cela suffit pour conserver la fraîcheur du sol et l'activité de la sève. 0 n doit pailler dès le 15 mai et même plus tôt dans les sols très-secs ou dans les climats chauds.

B I N A G E S

Pour que les plantes vivent et végètent avec vigueur, il faut que leurs racines puissent jouir des influences atmosphériques : l'air, l'humidité, la chaleur. Il est donc nécessaire que le sol soit meuble à la surface. Rien n'est plus propre que les binages qu'on fait à ou 6 centimètres de profondeur, pour l'aérer, conserverl'humidité et régulariser la chaleur. Il fautbri- 
ser la croûte du sol très-souvent avec la binette, afin d'obtenir ces effets.

\section{MASTIC A GREEFER A GHAUD}

L'arboriculteur a très-souvent recours au mastic à greffer. Il y en a de deux sortes. Ceux qu'on est obligé de faire chauffer pour les employer,ceux, au contraire, qui restent mous, dits mastics à froid. Voici la recette d'un mastic à chaud.

Poix noire. . . . . . 30 grammes.

Poix de Bourgogne . . . 26

Cire jaune. ...... 16 -

Suif ....... $14-$

Cire jaune ..... 14 -

100 grammes.

Le mastic à chaud est toujours préférable au mastic à froid quel qu'il soit.

MASTIG A GREFER A FROID

Poix blanche ou de Bourgogne. 20 grammes.

Résine........ 20 -

Téréberıchine...... 2 .

Cire ............ 15 -

Suif ......... 15 -

Cendre........ ऍ -

100 grammes. 
Ce mastic s'emploie à froid après l'avoir manié dans les mains qu'on se mouille légèrement pour empêcher qu il y adhère.

Le mastic à chaud et celui-ci sont très-propres à recouvrir les plaies des arbres.

Nous ne parlerons pas de l'onguent de saint Fiacre qui est abandonné maintenant, parce que ceux-ci sont de beaucoup préférables.

SULFATAGE DES TREILLAGES

Tout treillage en bois doit être sulfaté, alors même qu'on voudrait le faire peindre, car la peinture est loin de posséder la propriété de conserver le bois comme le sulfatage.

Rien n'est plus simple que de sulfater des lattes de treillage. Ayez un auget en bois, composé de trois planches assemblées aux extrémités et formant la longueur de votre treillage; placez-y vos lattes et versez dessus un liquide composé de :

Eau......... 100 litres.

Sulfate de cuivre .... 3 kilos.

Le sulfate de cuivre doit être dissous dans l'eau à l'avance.

En huit ou dix jours l'opération est complète, même pour des échalas et des tuteurs de quatre centimètres carrés. Si l'on est pressé, on peut retirer les 
lattes de treillage au bout de trois ou quatre jours.

L'eau sert jusqu'à épuisement complet. Cependant, quand arrive la fin, il est bon de remettre un peu de sulfate de cuivre.

Toutes les essences de bois gagnent à être sulfatées, depuis le bois le plus tendre jusqu'au bois le plus dur. Le bois sulfaté dure dix fois plus, environ, que celui qui ne l'est pas.

Grossissement Des fritts par le sulfatage

Quelques auteurs recommandent d'arroser les fruits avec une dissolution de sulfate de fer pour les faire grossir. Nous avouons n'avoir pas encore eu la curiosité de faire cet essai ; aussi ne pouvons-nous le recommander en toute assurance : nous le citons pour mémoire.

Voici en quoi consiste cette opération : on fait dissoudre 2 grammes de sulfate de fer dans 1 litre d'eau, et à l'aide d'une pompe à main on en bassine les fruits à trois fois différentes après le coucher du soleil; savoir : quand ils ont acquis le quart de leur grosseur, lorsqu'ils sont à moitié, et enfin, quand leur développement est complet.

Les fruits à pépins et d'été sont les seuls, à ce qu'il paraît, qui doivent recevoir ces aspersions.

Ce n'est ni difficile ni coûteux; que les amateurs essaient donc. 
LA B O OR S

Les labours du jardin fruitier doivent se faire au croc et non à la bêche, afin d'éviter de détruire le chevelu des arbres, ce qui leur cause un tort considérable. Le croc remue, ameublit la terre et la binette détruit les herbes, ce qui complète le travail. Mais les plates-bandes qui sont plantées de pyramides et d'espaliers et de plantes diverses ne peuvent s'accommoder de ce genre de labour; il est donc nécessaire de recourir à la bêche. Dans ce cas, il faut faire la plus grande attention pour que le labour ne dépasse pas 10 à 12 centimètres de profondeur autour du pied des arbres pour laisser toutes les racines intactes.

D N GHAULAGE

Le chaulage est de toute nécessité pour les arbres qui sont envahis par les insectes qui se réfugient dans la mousse et dans les interstices des écorces. On chaule les tiges et les branches charpentières avec un lait de chaux à consistance de bouillie trèsclaire qu'on applique à l'aide d'un pinceau ou brosse de peintre. Cette opération doit se faire de bonne heure au printemps ou à l'automne aussitôt que les feuilles sont tombées, souvent même avant et après l'hiver. 
Le chaulage est encore utile dans les expositions très-chaudes où les arbres souffrent de la chaleur. En les chaulant, les rayons solaires sont repoussés par la couleur blanche et ont moins d'influence.

C'est surtout pour les espaliers que le chaulage se pratique; mais on pourrait en étendre l'usage à tous les arbres quels qu'ils soient et quelle que soit la forme qu'ils aient, pyramides, espaliers, cordons ou haute tige, pour les débarrasser de la mousse quand ils en sont recouverts. 
Nous avons fait figurer au premier rang, pour les hautes tiges, Franceline Millot et Docteur Bouvier; cependant, certains arboriculteurs les classent en deuxième ordre. Si nous avons adopté ces deux variétés, c'est parce qu'elles sont vigoureuses et très-productives. Les fruits sont de bonne garde, assez bons crus et très-bons cuits. Du reste, quand le mois de mai arrive, on ne doit pas se montrer très-exigent sur la qualité des fruits : mieux vaut en avoir de passables que de n'en pas avoir du toul. Nous croyons donc qu'il est indispensable d'admettre, de préférence à beaucoup d'autres, ces deux variétés dans les vergers et dans la grande culture de spéculation.

Nous avons supprimé complétement le Doyenné d'hiver; mais l'amateur pourra encore lui sacrifier quelques coins, à une bonne exposition et dans un terrain exceptionnel; mais qu'il sache bien, à l'avance, qu'il nè doit pas compter sur ses fruits, à moins de circonstances très-favorables; il aura à peine trois bonnes poires sur une douzaine.

Depuis trente ans, nous nous occupons des bonnes variétés de poires, et nous avons acquis la conviction que fort peu de gens savent déguster; qu'un grand nombre ignorent la valeur des fruits d'hiver, ne savent pas en faire la différence avec ceux d'automne el d'été, et qu'il faut n'accueillir qu'avec réserve ce qui se dit et se publie à ce sujet par des gens inléressés à déguiser la vérité, ou n'ayant pas suffisamment étudié la question qui nous occupe.

Nous recommandons tout particulièrement à l'attention des amateurs la poire verte longue précoce de la Sarthe, bien que nous ne l'ayons pas classée définitivement faute d'étude suffisante. C'est, croyons-nous, l'un des meilleurs fruits d'été.

Nous avons négligé Van Mons de Léon Leclerc; cette poire se tavelle et se gerce, parfois; cependant, elle doit être étudiée et non abandonnée. C'est l'un des meilleurs fruits.

Nous avons conservé la calville blanche, faute d'autres pommes pour la remplacer; mais c'est une variété usée qui ne produit plus qu'en petite forme et dans des terrains exceptionnels. Peut-être la Pomme de Lestre la remplacera. - En général, ou attache trop d'importance à la pomme, sclon nous et beaucoup d'autres, c'est un fruit de consommation, plutôt qu'un fruit de dessert. Son acidité est du reste malfaisante et sa chair indigeste crue. 


\section{EXTRAIT' DU CATALOGUE}

DE S

ARBRES FRUITIERS, ASPERGES, FRAISIERS, VIGNES, ETC.

\section{DE \\ V.-F. LEBEUF}

HORTICULTEUR

A ARGENTEUIL (SEINP' HT-OISE)

Les commandes sont exactement remplies, et l'identité des variétés garantie. - Le Catalogue génèral est envoyé franco sur demande; il paraît, tous les ans, vers le 15 septembre.

\section{ARBRES FRUITIERS}

Les meilleures variétés pour la table. (Voir le catalogue pour les prix.)

Avis important. - Faute de désignation nous assortirons les variélés au mieux. Toute personne qui n'aurait pas de prédilection pour une variété plutôt que pour une autre, pourra s'en rapporter à notre choix qui sera toujours fait consciencieusement et de manière à comprendre des fruits d'été, d'automne et d'hiver de la plus longue durée, de première qualité, les plus fertiles, etc. 


\section{ASPERGES D'ARGENTEUIL}

\section{Asperge rouge ou violette hâtive d'Argenteuil.}

les 100 griffes, 1 er choix
le mille
les 100 griffes, 2 e choix

\section{Asperge rouge ou violette tardive d'A rgentemil.}

les 100 griffes, 1 er choix . . . $10 \mathrm{fr}$. le mille . . . , . . $90 \mathrm{fr}$. les 100 griffes, $2 \mathrm{e}$ choix . . . 7 fr. le mille . . . . . . $65 \mathrm{fr}$.

Ces variétés sont les plus belles et les plus estimées de toutes celles connues. Divers rapports de la Société Impériale Centrale d'Horticulture de France l'ont constaté. Elles ont obtenu près de cent récompenses et médailles tant en France qu'à l'étranger. Elles se plautent sans engrais, sans défoncement ni transport de terre, en février, mars et avril, au centre et au nord de la France; mais dans le midi on peut planter à l'automne.

L'asperge réussit dans tous les sols, pourvu qu'ils ne soient pas trop humides el qu'ils aient vingt-cinq centimètres de terre végétale.

Par l'ancienne méthode, il en coûtait plus de 100 francs (fosse, engrais et plantation) pour établir un carré de cent touffes d'asperges; aujourd'hui, ce travail se fait avec une journée d'homme (5 francs au plus). Un hectare de terre planté en asperges produit à Argenteuil de 6 à 7,000 fr. de bénéfice net. Voir la brochure : Les Asperges, les Fraises, les Figues et les Framboises, pour connaitre le mode de culture. 


\section{FRAISIERS}

La plus belle collection de fraisiers, composée de 280 variếtés à gros fruits, de race américaine, dont 100 de premier choix, et 10 variétés de race européenne dites des Quatri-Saisons ou remontantes.

(Demander le catalogue général et descriptif pour plus amples renseignements.)

\section{VIGNES POUR RAISINS DE TABLE}

Collection des cinquante meilleures variétés, en plantes hien enracinées.

Chasselas pour grandes plantations.

\section{FRAVBOISIERS}

Collection remarquable, composée des plus belles et des meilleures variétés.

Les variétés remontantes sont très-recherchées aujourd'hui, notamment celles qui fournissent le plus de fruits à l'automne (Consulter le catalogue général).

\section{GROSEILLIERS}

20 variétés les plus remarquables.

\section{NOISETIERS}

1 variêtés les plus recherchées.

\section{GLAIEULS}

Collection très-belle et très-variée par cent et par mille

Demander le Catalogue général descriptif et détaillé pour se renseigner sur les prix, à M. Lebeuf, horticulteur à Argenteuil (Seine-et-0ise). Il est adressé franco à ceux qui en font la demande. 


\section{OUVRAGES DE M. LEBEUF}

Culture des champignons de couche et de bois ot de la truffe, ou moyen de les multiplier, reproduire, accommoder, conserver, de reconnaltre les champiğnons sauvages comestibles, etc., 1 vol. in-18 jésus, avec 20 gravures, franco par la poste, $1 \mathrm{fr} .50$.

Culture de la Vigne, Guide du Vigneron et de l'Amateur de treilles, indiquant, mois par mois, les travaux à faire dans le vignoble et dans les jardins sur les treilles; la manière de planter, gouverner, dresser, cultiver la vigne d'après toutes les méthodes en usage en France, la guérir de ses maladies; suivie de l'Ö̈dium, ou moyen de le traiter et de le gućrir, 1 vol. in-18, avec 32 gravures, 2 fr. 50.

Ies Asperges, les Fraises, les Figues et les Framboises, ou description des meilleures méthodes de cullure, pour les obtenir en abondance et presque sans frais, suivi de la manière de les forcer pour avoir des primeurs et des fruits pendant l'hiver, du calendrier du cultivateur d'asperges, de fraisiers et de figuiers, indiquant, mois par mois, les Iravaux a faire dans les aspergeries, les fraisières et les figueries, 1 vol. in-18, avec 28 figures, franco par la poste, $1 \mathrm{fr} .60$.

\section{C'Horticulteur-gastronone. - BONS IÁEUMES RT BONA} FITUTS, ou choix des meilleures variétés de plantes polageres et arbres fruitiers, vignes, etc., à cultiver; moyens de conserver les fruits et légumes pendant l'hiver, suivis des $\mathbf{3 6 5}$ salades de l'ami Antoine, de la manière d'établir un jardin potager fruitier de produit, et du Calendrier de l'hor. ticulteur. 1 vol. in-18, 1 fr. 50, franco par la poste. (On peut envoyer 7 timbres-poste à 20 centimes non séparés, et un à dix centimes.)

IRévolution sgricole, ou moyen de faire des bénéfices en cullivant $l_{\text {es }}$ terres, 1 vol. in-18, 5 gravures dans le texte, $2 \mathrm{fr}$. franco par la poste.

Dans cet ouvrage, l'auteur expose un système complétement nouveau, basé sur l'expérience et dont les résultats sont certains. C'est le seul travail qui existe en ce genre. 


\section{TABLE DES MATIÈRES}

Pourquoi un livRe nouveau SUR les arbres frultiers....... V V

\section{PREMIĖRE PARTIE.}

Culture des arbres fruitiers, plantalion, choix des variétés

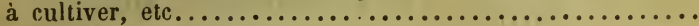

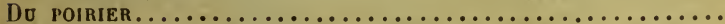

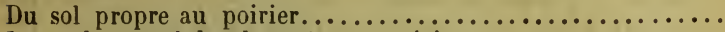

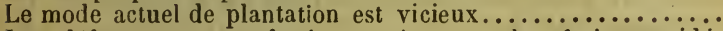

Les defoncements profonds occasionnent des frais considé-

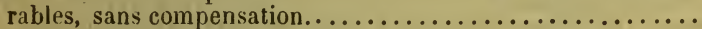

Les défoncements profonds sont-ils utiles? à quelle profondeur faut-il défoncer.........................

Défoncements rationnels et économiques................

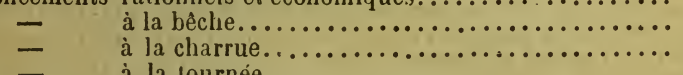

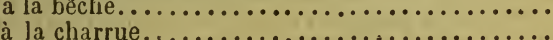

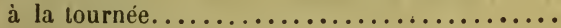

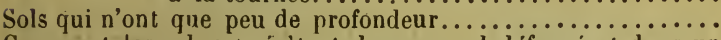

Comment les arbres végètent dans un sol défoncé et dans un sol qui ne l'èst pas; moyens de corriger les différances.....

Frais et produits des différents modes de plantation.........

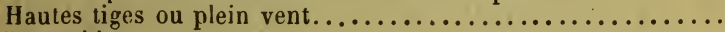

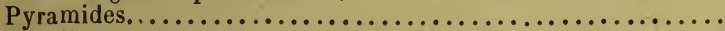

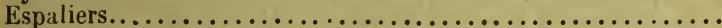

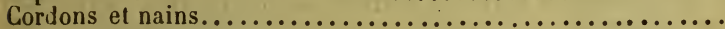

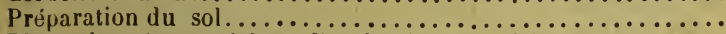

Plantation, époque à laquelle elle doit être faite............

Faut-il tailler les arbres lors de la plantation ?.............

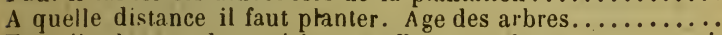

Faut-il planter des poiriers greffés sur franc ou sur coi-

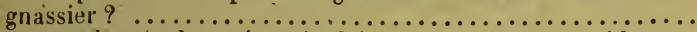

Doit-on planter des arbres a plein vent ou des pyramides....

Liste des trente variétés de poiriers les plus recommandables

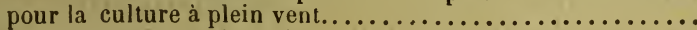

Liste des trente variétés les plus méritantes pour espaliers, pyramides, touffes et cordons par ordre de maturité et de

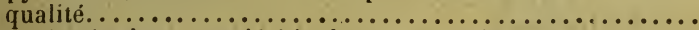

Quantité de chaque variété a planter en plein vent ou à haute

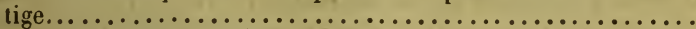

Quantite de chaque variété à planter en espalier et pyramides.

Description des meilleurs fruits.....................

Du poMmien . . . . . . . . . . . . . . . . . . . . . . .

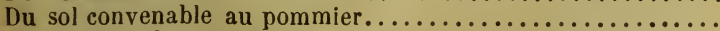

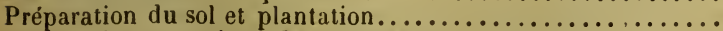

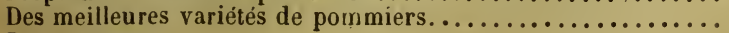

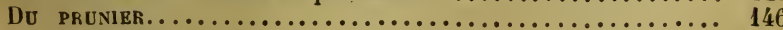

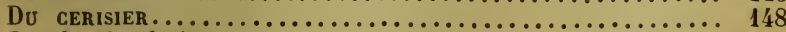

Récolte des fruits........................... 149 
Conservation des fruits......................... 151

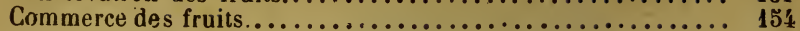

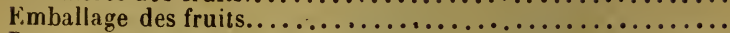

DE LA DÉGÉnÉRESCENCE et de l'extinction des végétaux en général et des races fruitières en particulier, nécessité de les remplacer.

\section{DEUXIĖME PARTIE.}

C'oneidérations générales sur la végétation et Notions DE PHYSIOI.OGIE VÉGÉTALE.........................

DE LA TAILLE. - De quoi se compose un arbre............... Effets des yeux à bois et des boutons à fruits sur la végétation Des productions fruitières ........................

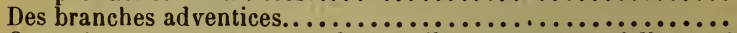
Opérations pratiquées lors de la taille ou après qu'elle a étè faite.

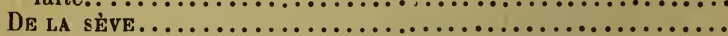

De la corrélation des racines avec les branches..............

Principes de la talle..............................

Moyen de répartir également la sève.....................

Treillage......................................

De la forme des arbres...........................

Taille du poirier à haute tige......................... - des arbres en touffes, en vase, en gobelet, en

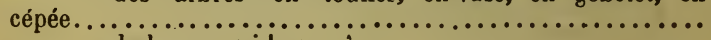
de la pyramide ou cóne.........................

Forme de l'espalier.

Taille de la palmette Verticale-Journet.................. Cordon horizontal..................................

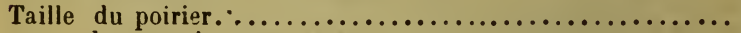

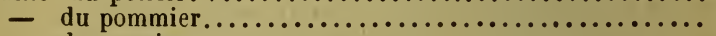

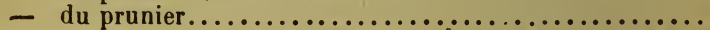

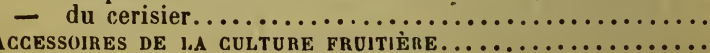

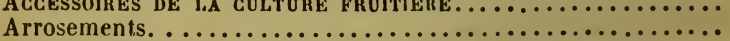

Bassinages. . . . .

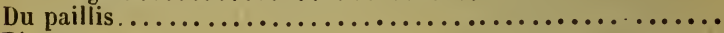

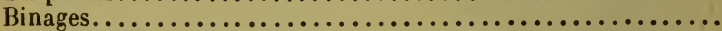

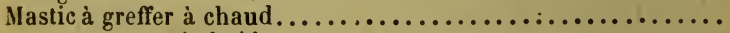

à froid.

Sulfatage des treillages...............................

Grossissement des fruits par le sulfatage...............

Labours.

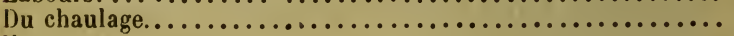

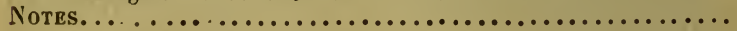

FIN DE LA TABLE DES MATIËIES. 


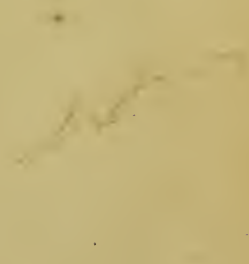





,



UNIVERSITY OF ILLINOIS-URBANA

634.2149 C001

ARBRES FRUITIERS

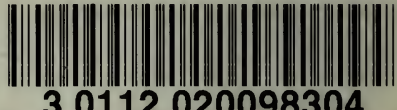

30112020098304 